ANL- 6855

Chemistry

(TID-4500, 33rd Ed.)

AEC Research and

Development Report

ARGONNE NATIONAL LABORATORY

9700 South Cass Avenue

Argonne, Illinois 60440

THE CRYSTAL STRUCTURE OF DISODIUM TETRANITRITONITROSOHY DROXYRUTHENATE(III)

2-HYDRATE BY NEUTRON DIFFRACTION

by

M. H. Muellex

Argonne National Laboratory

and

S. H. Simonsen

The University of Texas

Metallurgy Division

Final Report - Program 4.10.22

A portion of the material in this report has appeared in the following Metallurgy Annual Reports:

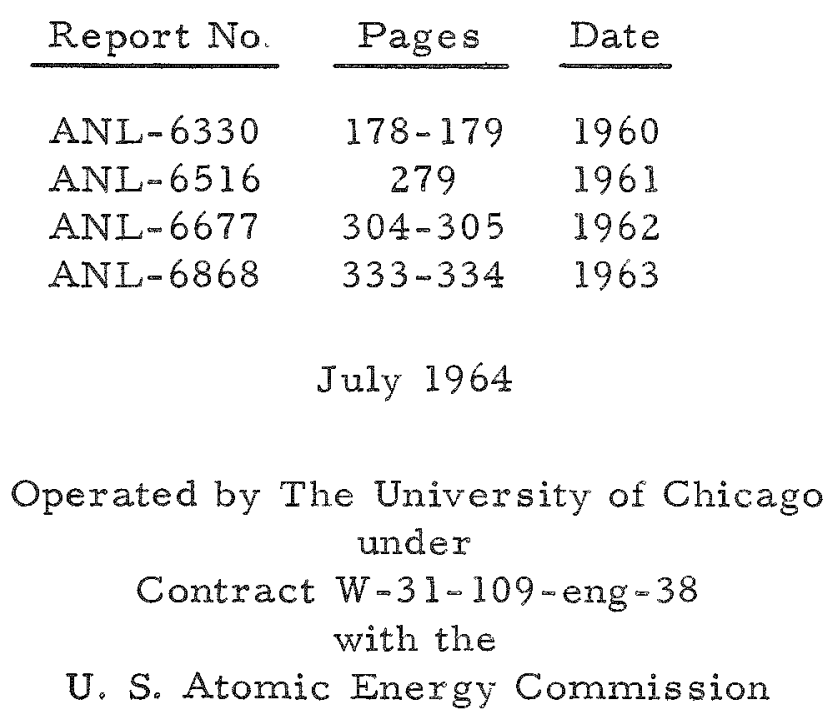




\section{DISCLAIMER}

This report was prepared as an account of work sponsored by an agency of the United States Government. Neither the United States Government nor any agency Thereof, nor any of their employees, makes any warranty, express or implied, or assumes any legal liability or responsibility for the accuracy, completeness, or usefulness of any information, apparatus, product, or process disclosed, or represents that its use would not infringe privately owned rights. Reference herein to any specific commercial product, process, or service by trade name, trademark, manufacturer, or otherwise does not necessarily constitute or imply its endorsement, recommendation, or favoring by the United States Government or any agency thereof. The views and opinions of authors expressed herein do not necessarily state or reflect those of the United States Government or any agency thereof. 


\section{DISCLAIMER}

Portions of this document may be illegible in electronic image products. Images are produced from the best available original document. 


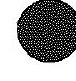

3 ,

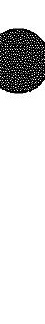

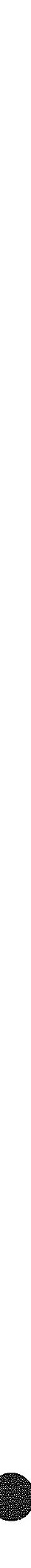




\section{TABLE OF CONTENTS}

Page

ABSTRACT ......................... 7

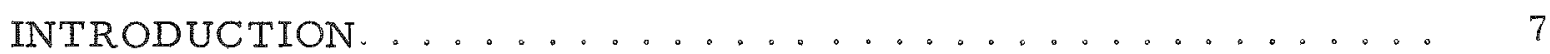

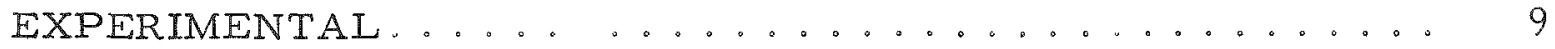

DETERMINATION OF THE STRUCTURE ........... 10

DISCUSSION OF THE STRUCTURE ................... 59

A. Configuration about the Ruthenium............ 60

B. Configuration about the Sodium-1 ............ 64

C. Configuration about the Sodium-2 ............ 65

D. General Features of the Structure............. 65

ACKNOWLEDGEMENTS ....................... 68

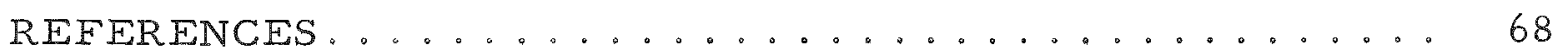




\section{LIST OF FIGURES}

No. Title

Page

1. Photo of Single Crystal Orienter as Positioned on the Argonne Neutron Spectrometer II ...............

2. Distribution for Intensities of $(0 \mathrm{k} l)$, (hol), and (hk0) Zones of Disodium Tetranitritonitrosohydroxyruthenate(III) 2-Hydrate Compared with Theoretical Curves for 1 and $\bar{l} \ldots \ldots 10$

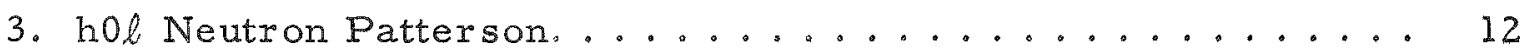

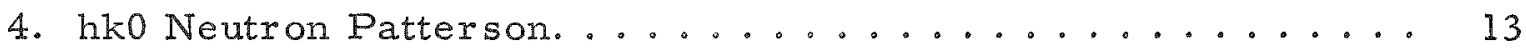

5. Okl Neutron Patterson................... 14

6. hol Neutron Patterson Showing Some of the Vector Distances. . 15

7. Neutron Fourier Showing Atom Positions on a 2-dimensional

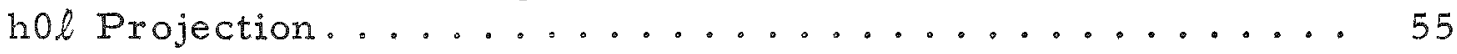

8. X-ray Fouriex Showing Atom Positions on a 2-dimensional

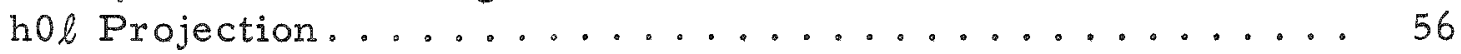

9. Section of hol 3-dimensional Neutron Fourier at $y=0$ Showing the Relative Location of HO-Ru-NO Groups. ...........

10. Lattice Constants and Schematic Structure of $\mathrm{Na}_{2}\left[\mathrm{Ru}\left(\mathrm{NO}_{2}\right)_{4} \mathrm{NO} \cdot \mathrm{OH}\right] 2 \mathrm{H}_{2} \mathrm{O} \ldots \ldots \ldots$

11. Octahedra Viewed Down the c Axis.............. 59

12. Octahedra Viewed Down the b Axis............... 59

13. Configuration about the Ruthenium Atom............60

14. Configuration about the Sodium-1 Atom ............664

15. Configuration about the Sodium-2 Atom .......... 65 


\section{LIST OF TABLES}

No.

Title

Page

I. Distribution of Reflections for Refinement ......... 15

II. Sequence of the Least-squares Refinement ......... 16

III. Final Coordinates and Errors of all Atoms from Final

Least-squares Results .................. 17

IV. Final Thermal Parameters $B_{i j} \ldots \ldots \ldots 17$

V. Table of Fcalc and Fobs for all Reflections after Final

Least-squares Cycle Based on ABC Reflections........

VI. Comparison of Atom Locations Based on the Fcalc

Fourier, Fobs Fourier, and the Least-squares Results

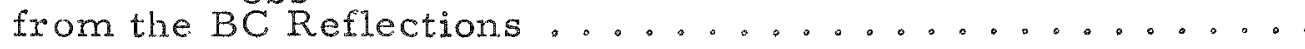

VII. Effect of the Missing Reflections as Indicated by a Comparison of Atom Locations Based on an F calc Fourier with and without These Reflections ..............

VIII. Some Inter - and Intramolecular Bond Distances and Angles. .

IX. RMS Radial Thermal Displacement and the RMS Component

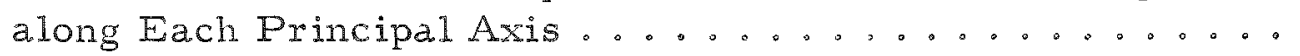



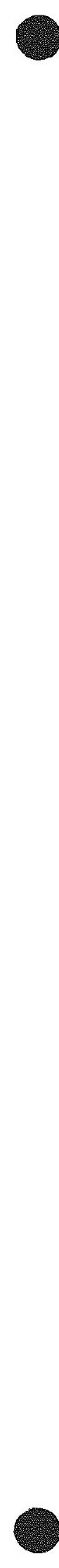

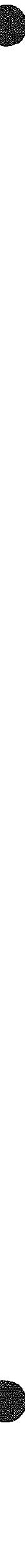




\title{
THE CRYSTAL STRUCTURE OF DISODIUM TETRANITRITONITR OSOHYDROXYRUTHENATE(III) 2-HYDRATE BY NEUTRON DIFFRACTION
}

\author{
by \\ M. H Mueller \\ Argonne National Laboratory \\ and \\ S H, Simonsen* \\ The University of Texas
}

\begin{abstract}
The crystal structure of $\mathrm{Na}_{2}\left[\mathrm{Ru}\left(\mathrm{NO}_{2}\right)_{4}(\mathrm{NO})(\mathrm{OH})\right] \cdot 2 \mathrm{H}_{2} \mathrm{O}$ was investigated by neutron diffraction, and the positional parameters of the heavy and light atoms weredetermined. The unit cell is monoclinic: $a_{0}=12.75 ; b_{0}=14.52 ; c_{0}=7.37 \AA$; $\beta=121.20^{\circ}$. The space group is $\mathrm{C} 2 / \mathrm{m}$, with 4 molecules (100 atoms) per unit cell and systematic absences are $\mathrm{h}+\mathrm{k}=$ $2 n+1$. The structure can be considered as consisting mainly of 6-fold coordination about three central atoms, namely, $R$ u, $\mathrm{Na}_{1}$, and $\mathrm{Na}_{2}$, forming three different kinds of octahedra. These octahedra form an interlocking chain throughout the structure, in which common atoms or common edges are shared between the octahedra. The 6-fold coordination about the ruthenium consists of four nitrogens of the nitrito groups arranged in a square, the nitrogen of the nitroso, and the oxygen of the hydroxyl group. These ruthenium octahedra are held together by $\mathrm{Na}-\mathrm{O}$ bridges involving 6-fold coordination about the two crystallographically different sodium atoms. Hydrogen bonding apparently is not important in this crystal.
\end{abstract}

\section{INTRODUCTION}

The general features of the stereochemistry of the coordination complexes of ruthenium have been established by the structure determinations of several compounds: (a) $\mathrm{K}_{4} \mathrm{Ru}_{2} \mathrm{Cl}_{30} \mathrm{O}_{0}$ (1) (b) $\mathrm{K}_{2} \mathrm{RuCl}_{6^{\circ}}$ (2) (c) $\left[\mathrm{Ru}\left(\mathrm{NH}_{3}\right)_{4}\right.$ (NO) $(\mathrm{OH})] \mathrm{Cl}_{2} ;(3)(\mathrm{d})\left(\mathrm{NH}_{4}\right)_{2}\left[\mathrm{RuCl}_{4}(\mathrm{NO})(\mathrm{OH})\right]_{;}(4)$ and $(\mathrm{e}) \mathrm{K}_{2}\left[\mathrm{Ru}\left(\mathrm{NO}_{2}\right)_{4}(\mathrm{NO})(\mathrm{OH})\right]_{0}(5)$

*Resident Research Associate Summer of 1960 and 1962, Consultant to the Metallurgy Division. 
However, there were some details in the structure of (c) and (d), especially the $\mathrm{Ru}-\mathrm{N}-\mathrm{O}$ bonding, that made it desirable to examine a similar type of compound, particularly in view of the current interest in ruthenium nitrosyl complexes. Also, all the previous structures had been solved by projections and the more accurate bond lengths attainable from three-dimensional data were desired. The structure of $\mathrm{Na}_{2}\left[\mathrm{Ru}\left(\mathrm{NO}_{2}\right)_{4}(\mathrm{NO})(\mathrm{OH})\right] \cdot 2 \mathrm{H}_{2} \mathrm{O}$ was determined by neutron diffraction for several reasons:

(1) We wished to demonstrate the power of the neutron diffraction method in the complete solution of a fairly complex structure, applying the standard techniques used in $\mathrm{X}$-ray determinations. No X-ray work had been done on the compound and its structure was unknown at the start of the investigation. At a later point in the work, some X-ray zone intensity datawere collected to illustrate the difference in the $\mathrm{X}$-ray and neutron Fouriers.

(2) Because ruthenium contributes so greatly to the scattering in the case of $\mathrm{X}$ rays, the accuracy of the light-atom parameters is affected. In the case of neutrons, the scattering contribution by all atoms is more nearly equal, so that all atoms are located with about equal precision.

(3) Information concerning the role of possible hydrogen bonding required knowledge of the hydrogen positions, attainable best by neutron diffraction.

The preliminary results of this investigation have been reported in the Metallurgy Division Annual Reports listed on the title page. A summary was also presented orally at the International Union of Crystallography Sixth International Congress held in Rome, Italy, September 9-18, 1963 and was published in their Abstracts. (6) A paper (7) has also been prepared and submitted for publication. Howevex, since it was not possible or planned to include in the above publications many of the important details of the experimental technique or the listing of the final agreement between the observed and calculated structure factors of approximately 2000 reflections, this report has been prepared. 


\section{EXPERIMENTAL}

Pollack and Wallace ${ }^{(8)}$ prepared large crystals in the following way: Sodium nitrite, slightly in excess of the stoichiometric amount, was added to an aqueous solution of ruthenium(IV) chloride. The solution was heated until a red color developed and was then allowed to cool slowly. The crystals were large, well-formed, reddish-orange prisms.

The unit-cell dimensions were obtained conventionally from rotation, Weissenberg, and precession X-ray photographs. The cell was monoclinic, with the following dimensions: $a=12.75 ; b=14.52 ; c=7.37 \AA$; $\beta=121.20^{\circ}$. The systematic absences $(h+k=2 n+1)$ indicated the possible space groups $\mathrm{C} 2, \mathrm{Cm}$, or $\mathrm{C} 2 / \mathrm{m}$. The density, measured by displacement of carbon tetrachloride, was found to be $2.29 \mathrm{~g} \mathrm{~cm}^{-3}$. The calculated density with $\mathrm{z}=4$, is $2.36 \mathrm{~g} \mathrm{~cm}^{-3}$.

A single crystal, in the form of a cube $0.46 \mathrm{~cm}$ on an edge, was positioned on a GE Single Crystal Orienter mounted on Neutron Spectrometer II at the Argonne CP-5 reactor, as shown in Figure 1. A neutron wavelength of $1.16 \AA$, reflected from the (111) face of a copper single crystal, was used. The neutron flux at the sample was approximately $10^{6}$ neutrons $\mathrm{cm}^{-2} \mathrm{sec}^{-1}$.

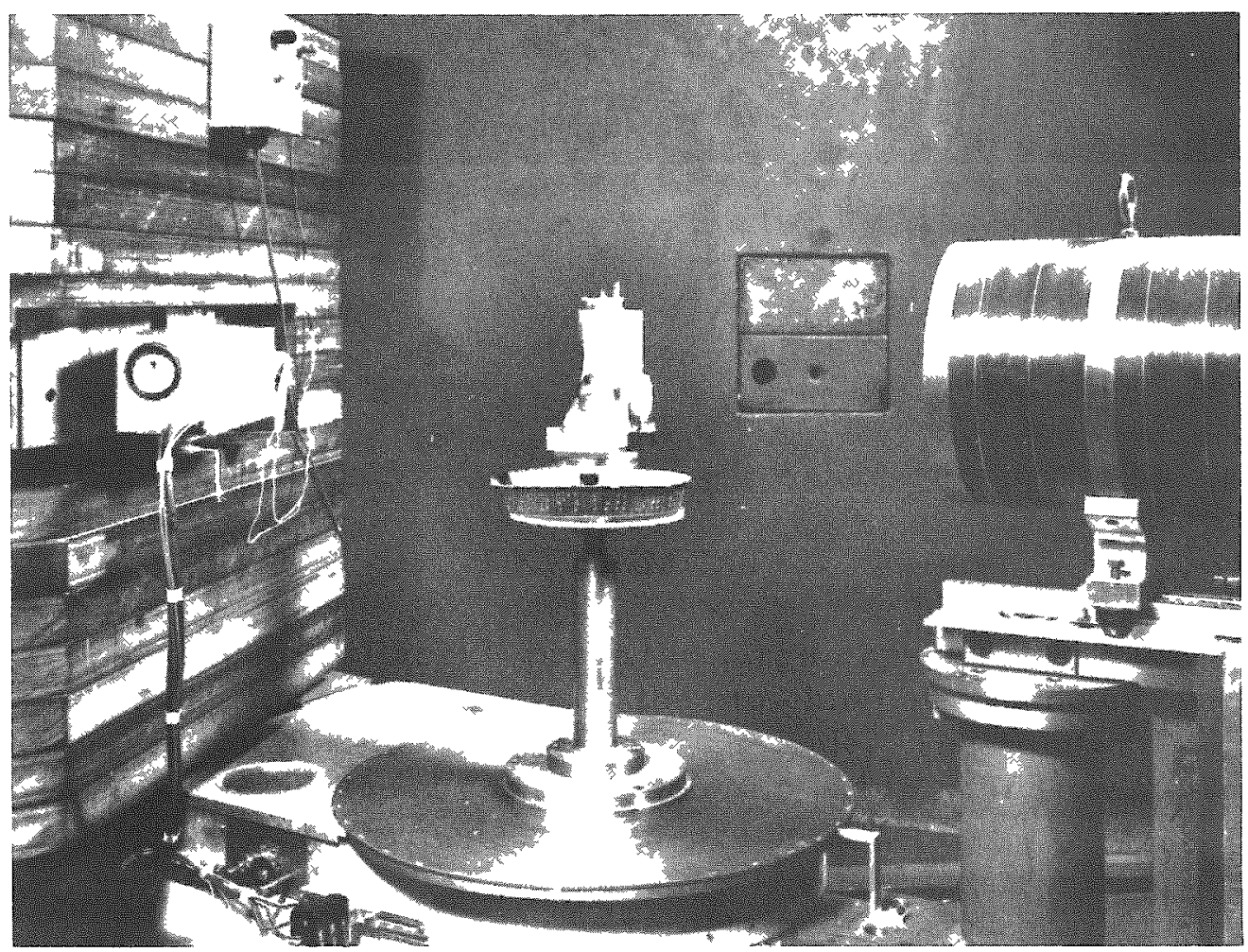

$106-5801$

Fig. 1. Photo of Single Crystal Orienter as Positioned on the Argonne Neutron Spectrometer II 
Integrated intensities were obtained by a $\theta-2 \theta$ scan after phi and chi angles on the orienter had been set manually. The primary beam was monitored with a fission counter acting as the timing device for the counting intervals. The scaler count and angle were printed out on a typewriter at each interval of $0.10^{\circ} 2 \theta$ and the scaler reset. Integrated intensity $=$ ( $\Sigma$ interval counts) - (number of intervals $x$ average background per interval). These integrated intensities were multiplied by the Lorentz correction $(\sin 2 \theta)$ to obtain $F^{2}$ values. No correction was made for absorption because the transmission was high (approximately $80 \%$ ) and the sample was symmetrically shaped. A more detailed description of this instrument and the techniques used is given in an ANL report. (9)

Integrated intensities of 1359 reflections were measured: 567 more were below the limit of measurement. Another 301 reflections were available in the sphere of reflection, but unfortunately the crystal was destroyed accidentally before measurements were completed.

\section{DETERMINATION OF STRUCTURE}

The statistical test of Howells et al. (10) was applied to the intensities of the (hk0), (hol), and ( $0 k l)$ zones, the range $\sin \theta=0-0.2$ being disregarded. The results, shown in Figure 2, indicate the space group to be $\mathrm{C} 2 / \mathrm{m}$, also suggested by the morphology of the crystal.

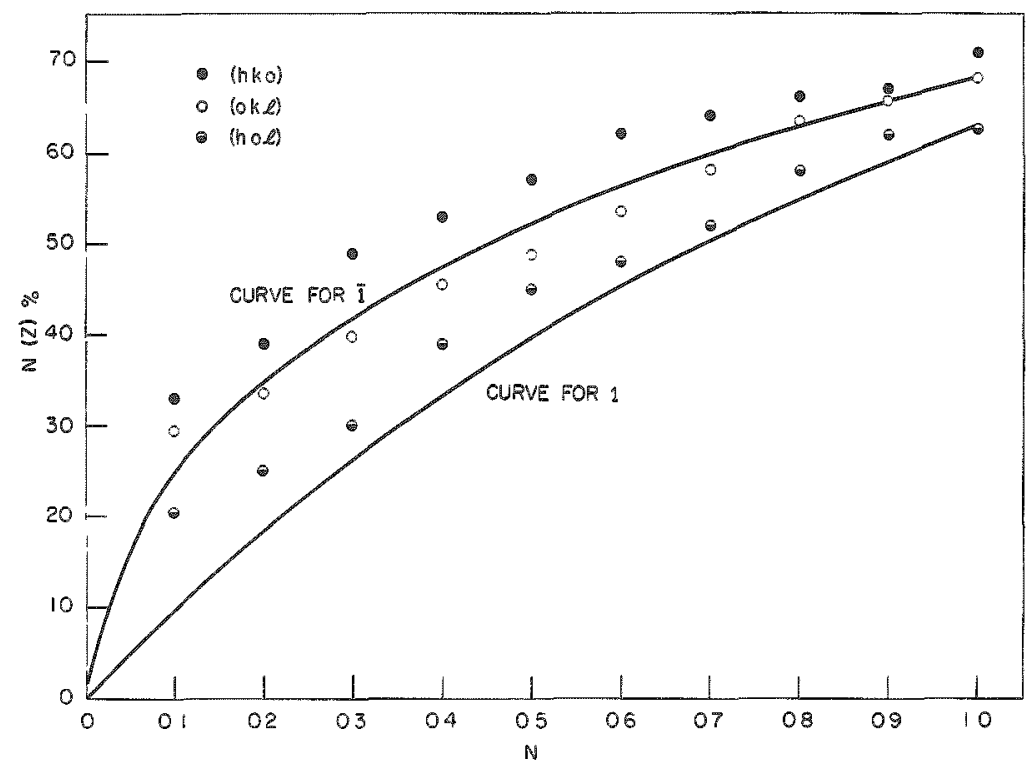

$106-5674$

Fig. 2. Distribution for Intensities of $(0 \mathrm{k} l)$, (hol), and (hko) Zones of Disodium Tetranitritonitrosohydroxyruthenate(III) 2-Hydrate Compared with Theoretical Curves for 1 and $I$ 
Howells, Phillips, and Rogers (10) derived distribution functions of intensities for noncentrosymmetric and centrosymmetric crystals, and showed that the percentage, $N(z) \%$, of the reflections whose intensities are less than or equal to $z$ ( = I/. I >), a fraction of the local average intensity, is given by:

$$
\begin{aligned}
& N(z) \%= 1-\exp (-z) \cdot 100 \text { for a noncentrosymmetric crystal, and } \\
& N(z) \%= \operatorname{erf}(z / 2)^{1 / 2} \cdot 100 \text { (where erf is the error function) for a } \\
& \text { centrosymmetric crystal. }
\end{aligned}
$$

To detect the presence of a center of symmetry, the experimental data are compared graphically with the theoretical curves in the following way.

Because the observed intensities fall off rapidly in I> with increasing $\sin \theta$ due to thermal motion in the case of neutron scattering, and to thermal motion and the form factors in the case of $X$-ray scattering, only the intensities within a small range of $\sin \theta$ are compared. The reflections and their intensities are arranged in order of increasing $\sin \theta$, weighting each reflection with the appropriate multiplicity. Systematic absences are not included, but accidental absences are given intensities of zero and included in the tabulation. Reflections having $\sin \theta$ less than 0.200 are not used.

The reflections are divided into several groups, each group containabout about an equal number of reflections, and the average intensity (I) determined for each group. The value of $N(z)$ for $z=0.1$ is obtained by counting the number of reflections having intensities less than or equal to $0.1<I>$ and dividing by the total number of reflections in the group. Values $N(0.2), N(0.3), \ldots, N(1.0)$ are found in a similar way. Each group is treated separately; the corresponding $N(z)$ values are then averaged for plotting, as in Figure 2 ( $N$ in Figure 2 corresponds to $z$ ).

Patterson projections, showing well-resolved peaks, were first computed from the neutron data of the three axial zones. At the time in 1960 that these zone data were collected the Pittsburgher 2-dimensional program(11) for the IBM-650 was the only working Fourier program available to us. The results obtained for the hol, hko, and $0 k l$ zones are shown in Figures 3,4, and 5, respectively. A number of the peaks in these Patterson maps were tentatively identified, especially in hol projection. Distances observed suggested that the $\mathrm{Ru}-\mathrm{N}\left(\mathrm{NO}_{2}\right)$ vectors were not in the ho $\ell$ plane but probably $45^{\circ}$ to it.

Since to our knowledge the Patterson function had not been used previously with neutron data and since the results from the sharp peaks shown above were initially encouraging, we continued to gather data for 


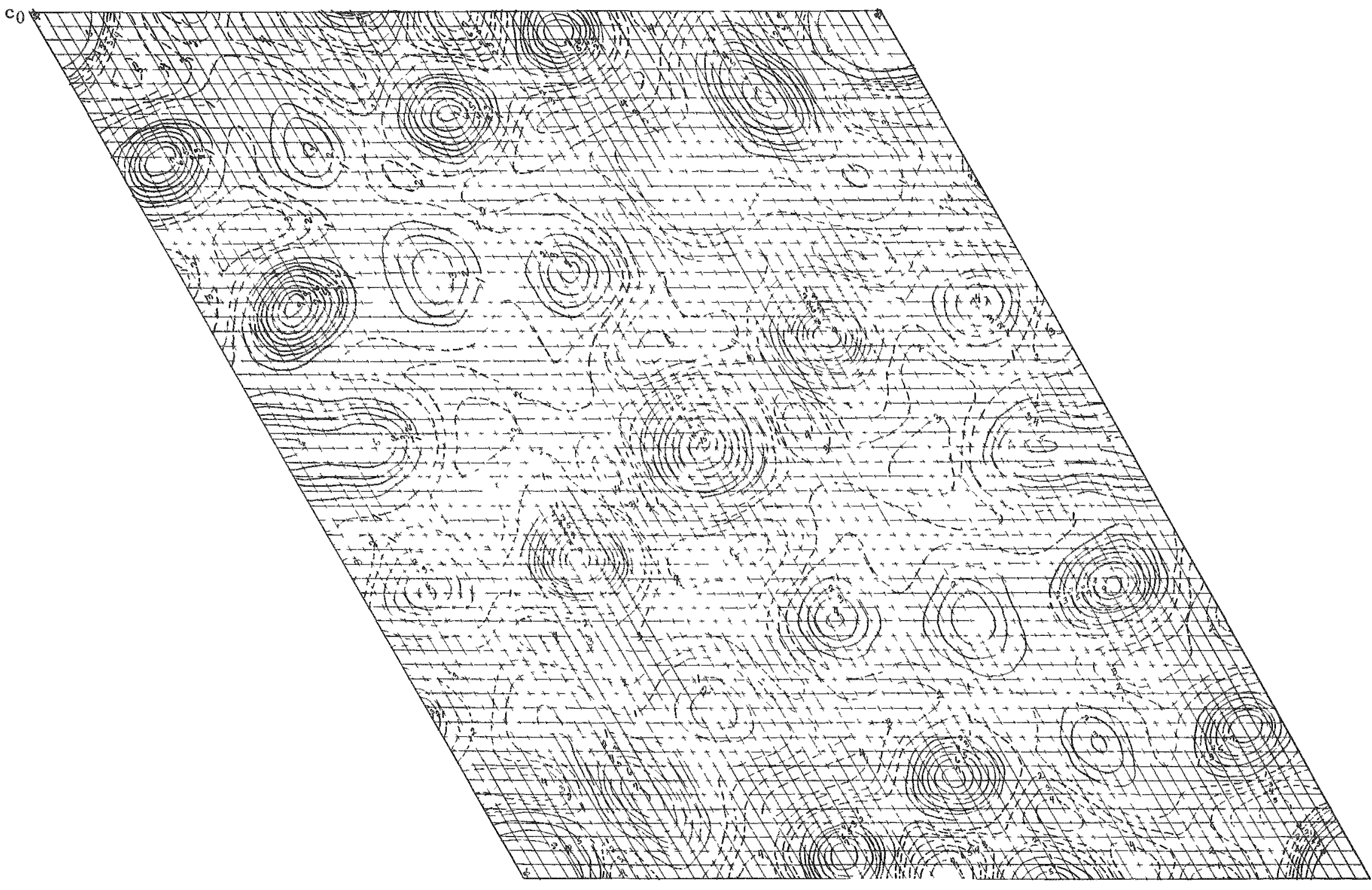

$106-5683 \mathrm{~A}$

Fig. 3. hol Neutron Patterson 
$b / 2$

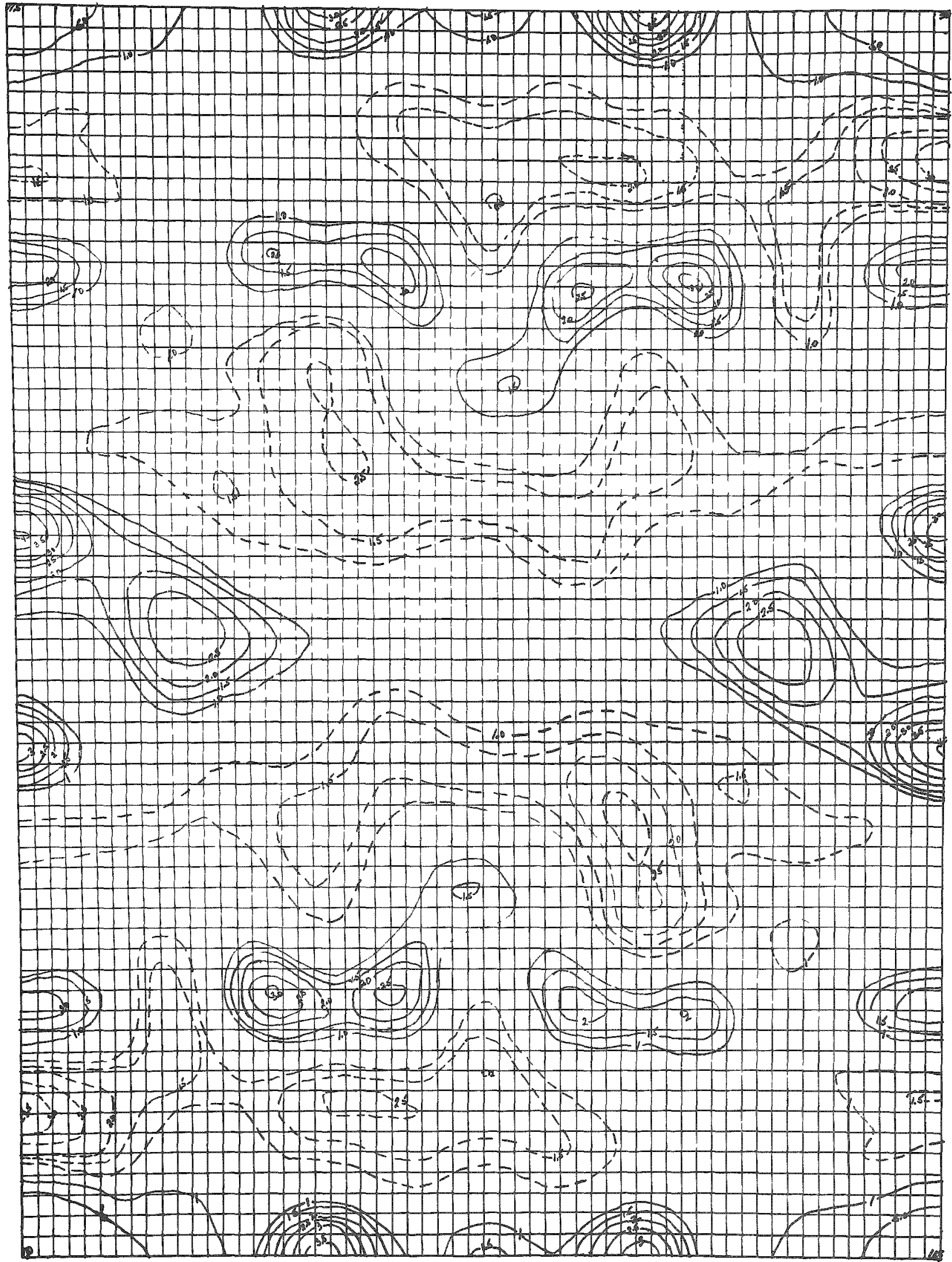




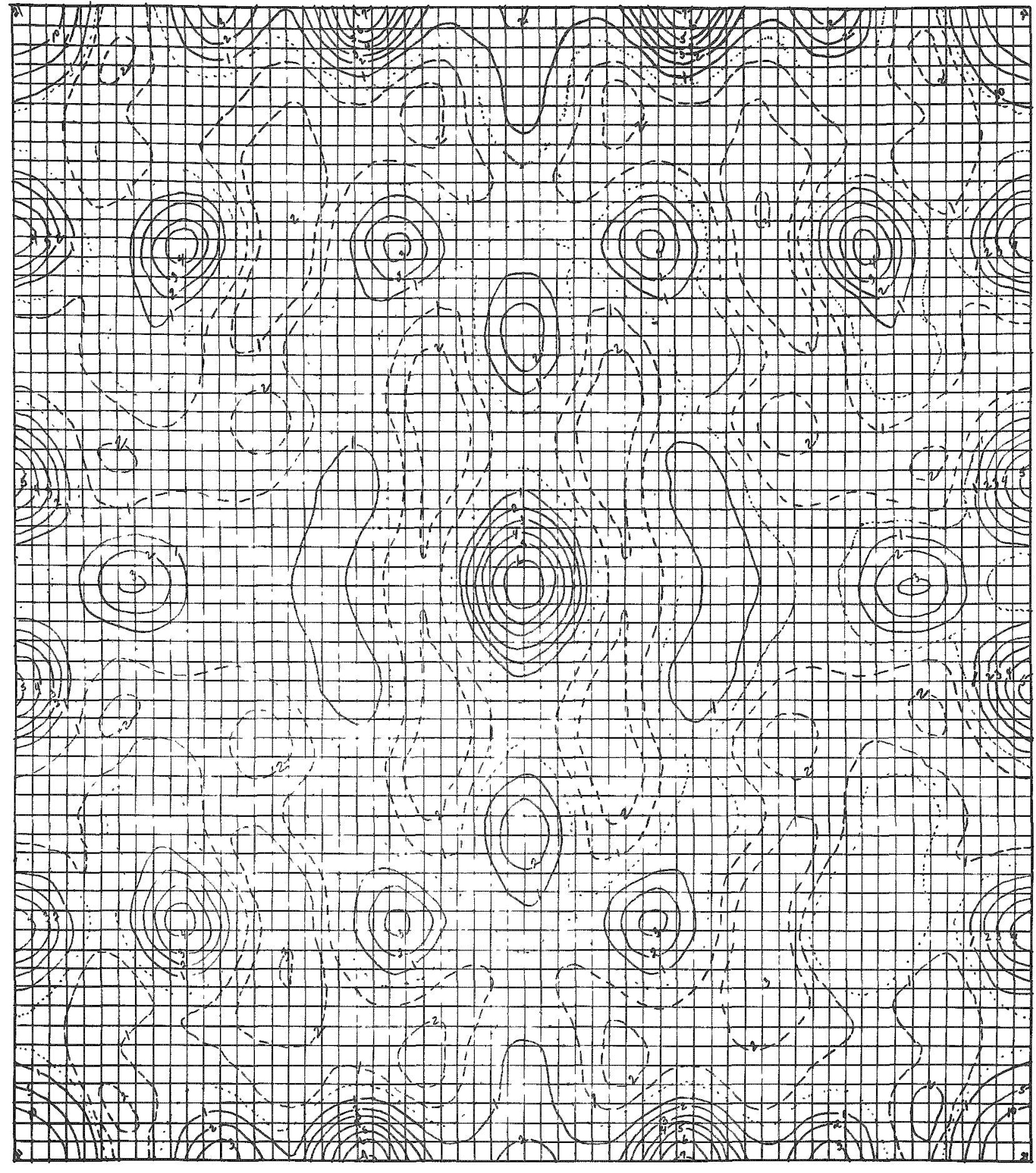
0 
a 3-dimensional Patterson. In the meantime, we obtained the Sly-Shoemaker 3-dimensional Fourier Program (12) for use with the IBM-704 computer. As a check on its successful operation we again ran the h0l 2-dimensional data. The results replotted from the computer output together with some of the vector identities as finally determined are shown in Figure 6. Some of the shorter vectors, such as the $R u-N, N-N$, and the short $N-O$, gave the initial hints of the structure.

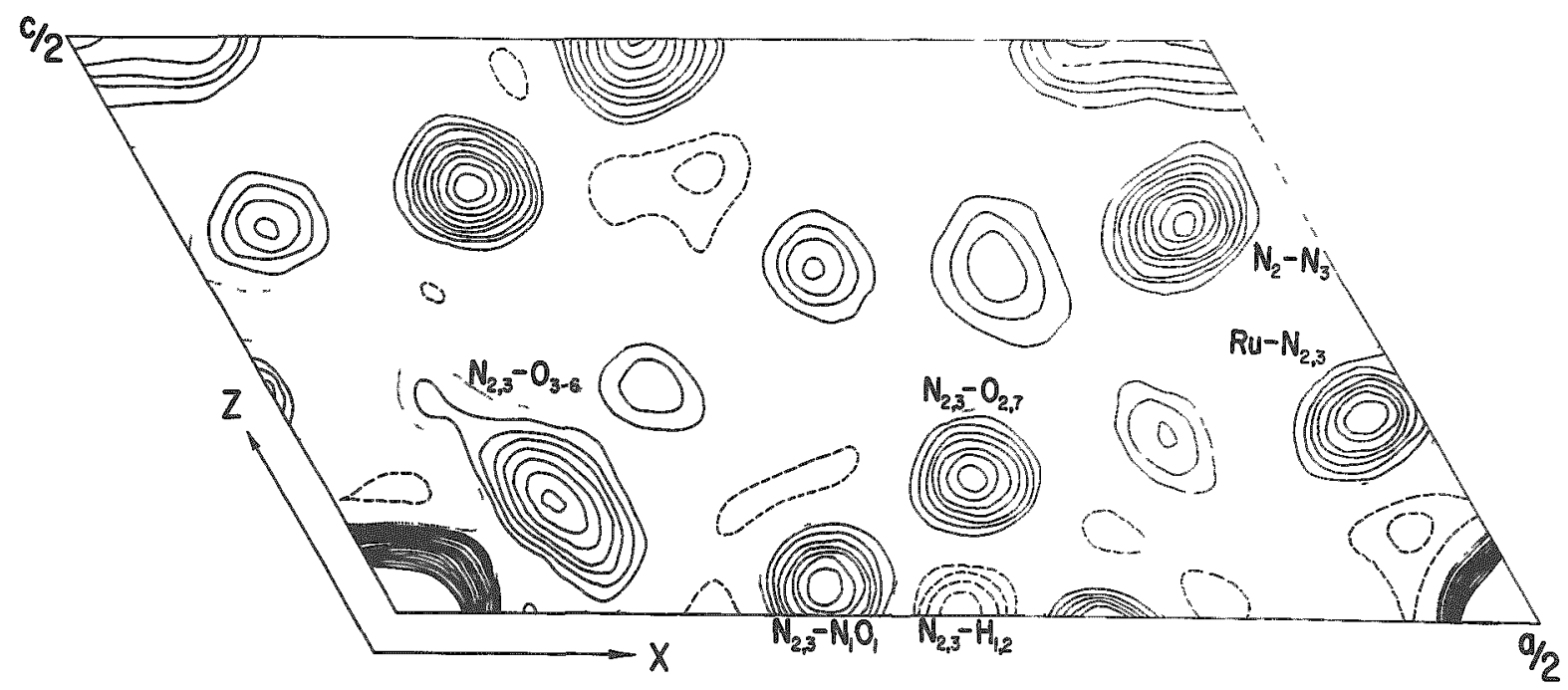

38521

Fig. 6. hol Neutron Patterson Showing Some of the Vector Distances

The 3-dimensional Patterson, calculated by means of above MIFRI computer program, also suggested the space group to be $\mathrm{C} 2 / \mathrm{m}$, and subsequent refinements were based upon this choice. The nitrito nitrogen vectors $\left(\mathbb{N}_{2}-\mathbb{N}_{3}\right.$ vectors) were readily identified in this 3 -dimensional map and used as image points for a superposition map based on the Buerger minimum function. (13) This was carried out on the IBM-704 by using the Penfold program. (14)

The trial structure thus obtained was refined by successive " $F_{0}$ " and " $F$ " synthesis until all atoms had been located. At this point refinement was continued by use of the Busing-Levy least-squares program. (15) To conserve computer time the reflections were divided into four groups according to Table I, and the refinement was carried out in progressive stages, as outlined in Table II.

Table I

DISTRTBUTION OF REELECIIONS FOR REFINEMEN]

\begin{tabular}{|c|c|c|}
\hline Group & Number of Roflections & Sin $\theta=$ Range \\
\hline A & 374 & $0-0.1990$ \\
B & 585 & $0.1993-0.3764$ \\
C & 400 & $0.3765-0.5827$ \\
D & 567 & Unobservea \\
\hline
\end{tabular}


Table II

SEQUENCE OF THE LEAST-SQUARES REFINEMENT

\begin{tabular}{|c|c|c|c|c|}
\hline & Parameters Refined* & $\begin{array}{c}\text { Reflections } \\
\text { Used }\end{array}$ & $\begin{array}{l}\text { Number } \\
\text { of Cycles }\end{array}$ & Final $R$ \\
\hline $\begin{array}{l}1 . \\
2 . \\
3 . \\
4 . \\
5 . \\
6 .\end{array}$ & $\begin{array}{l}\text { S.F., P, T } T_{0} \text { held constant } \\
\text { S.F., P, T0 } \\
\text { S.F., P, B } \\
\text { S.F., P, B } \\
\text { S.F., P, } B_{i j} \\
\text { S.F., P, } B_{i j}\end{array}$ & $\begin{array}{c}\mathrm{C} \\
\mathrm{C} \\
\mathrm{C} \\
\mathrm{BC} \\
\mathrm{BC} \\
\mathrm{ABC}\end{array}$ & $\begin{array}{l}6 \\
2 \\
1 \\
3 \\
3 \\
2\end{array}$ & $\begin{array}{l}0.364 \\
0.377 \\
0.193 \\
0.082 \\
0.098 * *\end{array}$ \\
\hline & $\begin{array}{l}\text { * S.F.: Scale factor } \\
P \text { : Positional parame } \\
T_{0}: \text { Overall temperatu } \\
B \text { : Individual isotropi } \\
B_{i j}: \text { Individual anisotro }\end{array}$ & $\begin{array}{l}\text { ps } \\
\text { parameters } \\
\text { temperature } \\
\text { ic }\end{array}$ & rameters & \\
\hline
\end{tabular}

The final calculation of the structure factor, based on individual anisotropic temperature parameters, gave an agreement index, $R=$ $\Sigma\left(\left|F_{0}\right|-\left|F_{C}\right|\right) / \Sigma\left|F_{0}\right|$, of $9.8 \%$ for the observed reflections, excluding the fol-

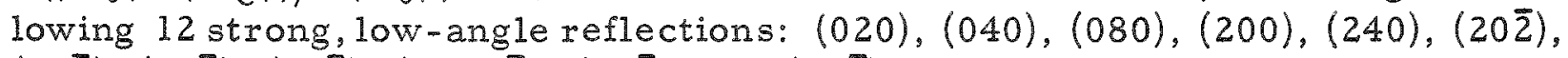
$(24 \overline{2}),(40 \overline{2}),(20 \overline{3}),(10,0, \overline{3}),(60 \overline{5})$, and $(40 \overline{4})$. It can be noted in Table V that these reflections do show low observed $F^{\prime}$ s relative to the calculated. The slightly lower $R$ of $8.2 \%$ shown in Table II for the BC reflections is probably due to the fact that the $A$ reflections, which have the lower value of $\sin \theta / \lambda$, generally do suffer from some extinction, and hence their inclusion increases the $R$ to the $9.8 \%$. When all of the unobserved reflections were included in the calculation, $R=17.7 \%$ was obtained. Some of this increase is probably due to errors in angle settings of the unobserved reflection ions.

The final least-squares positional parameters from the $A B C$ set are given in Table III, and the anisotropic temperature parameters are shown in Table IV. A list of observed and calculated structure factors obtained from the final cycle based on the $A B C$ set of reflections are shown in Table $V$. It should be noted that an asterisk in front of certain reflections in this table indicates that intensity measurements were not obtained since they were the missing reflections referred to previously. 
Table III

FINAL COORDINATES AND ERRORS* OF ALL ATOMS FROM LEAST -SQUARES RESUITS

\begin{tabular}{|c|c|c|c|}
\hline Atom & $\mathrm{X}$ & $\mathrm{Y}$ & $\mathrm{Z}$ \\
\hline $\mathrm{H}_{1}$ & $0.0449(8)$ & 0 & $0.8606(15)$ \\
$\mathrm{H}_{2}$ & $0.0086(21)$ & $0.2080(16)$ & $0.1811(22)$ \\
$\mathrm{H}_{3}$ & $0.4631(15)$ & $0.2190(8)$ & $0.2675(29)$ \\
$\mathrm{Ru}$ & $0.2623(3)$ & 0 & $0.0035(5)$ \\
$\mathrm{N}_{1}$ & $0.4197(3)$ & 0 & $0.1892(4)$ \\
$\mathrm{N}_{2}$ & $0.2311(2)$ & $0.1002(1)$ & $0.1715(3)$ \\
$\mathrm{N}_{3}$ & $0.2835(2)$ & $0.1024(1)$ & $0.8276(3)$ \\
$\mathrm{O}_{1}$ & $0.0875(4)$ & 0 & $0.7910(6)$ \\
$\mathrm{O}_{2}$ & $0.4788(5)$ & 0 & $0.6911(11)$ \\
$\mathrm{O}_{3}$ & $0.1289(5)$ & $0.1093(5)$ & $0.1420(9)$ \\
$\mathrm{O}_{4}$ & $0.3147(5)$ & $0.1474(4)$ & $0.3021(8)$ \\
$\mathrm{O}_{5}$ & $0.2122(4)$ & $0.1118(4)$ & $0.6391(6)$ \\
$\mathrm{O}_{6}$ & $0.3686(7)$ & $0.1552(6)$ & $0.9151(9)$ \\
$\mathrm{O}_{7}$ & $0.0121(5)$ & $0.2304(3)$ & $0.3021(8)$ \\
$\mathrm{Na}_{1}$ & 0.2500 & 0.2500 & 0.5000 \\
$\mathrm{Na}_{2}$ & 0 & $0.1098(6)$ & 0.5000 \\
\hline
\end{tabular}

*Standard errors $\left(\times 10^{4}\right)$ appear in parentheses.

Table IV

FINAL THERMAL PARAMETERS $B_{i j}$

\begin{tabular}{|l|c|c|c|c|c|c|}
\hline Atom & $B_{11}$ & $B_{22}$ & $B_{33}$ & $B_{12}$ & $B_{13}$ & $B_{23}$ \\
\hline$H_{1}$ & 0.0052 & 0.0109 & 0.0200 & 0 & 0.0070 & 0 \\
$H_{2}$ & 0.0322 & 0.0160 & 0.0336 & 0.0039 & 0.0223 & 0.0054 \\
$H_{3}$ & 0.0201 & 0.0046 & 0.0713 & -0.0035 & 0.0162 & -0.0053 \\
$R u_{1}$ & 0.0037 & 0.0021 & 0.0105 & 0 & 0.0034 & 0 \\
$\mathrm{~N}_{1}$ & 0.0041 & 0.0034 & 0.0136 & 0 & 0.0030 & 0 \\
$\mathrm{~N}_{2}$ & 0.0067 & 0.0028 & 0.0151 & 0.0004 & 0.0060 & -0.0009 \\
$\mathrm{~N}_{3}$ & 0.0055 & 0.0027 & 0.0158 & -0.0003 & 0.0055 & 0.0010 \\
$\mathrm{O}_{1}$ & 0.0039 & 0.0032 & 0.0128 & 0 & 0.0037 & 0 \\
$\mathrm{O}_{2}$ & 0.0044 & 0.0071 & 0.0246 & 0 & 0.0014 & 0 \\
$\mathrm{O}_{3}$ & 0.0080 & 0.0080 & 0.0333 & 0.0013 & 0.0102 & -0.0050 \\
$\mathrm{O}_{4}$ & 0.0103 & 0.0048 & 0.0298 & -0.0020 & 0.0108 & -0.0063 \\
$\mathrm{O}_{5}$ & 0.0077 & 0.0056 & 0.0176 & -0.0006 & 0.0041 & 0.0045 \\
$\mathrm{O}_{6}$ & 0.0167 & 0.0095 & 0.0228 & -0.0092 & 0.0070 & -0.0002 \\
$\mathrm{O}_{7}$ & 0.0124 & 0.0042 & 0.0233 & 0.0019 & 0.0105 & 0.0029 \\
$\mathrm{Na}_{1}$ & 0.0196 & 0.0026 & 0.0449 & -0.0004 & 0.0253 & -0.0001 \\
$\mathrm{Na}_{2}$ & 0.0049 & 0.0029 & 0.0251 & 0 & 0.0024 & 0 \\
\hline
\end{tabular}


Table V

TABLE OF $F_{\text {calc }}$ AND Fobs FOR ALL REFLECTIONS AFTER FINAL LEAST-SQUARES CYCLE BASED ON ABC REFLECTIONS

\begin{tabular}{|c|c|c|c|c|c|}
\hline$H$ & $k$ & L & CBSERVED & CALCULATED & OAS=CAL \\
\hline 0 & 2 & 2 & 4.522 & 0.223 & -1.709 \\
\hline 0 & 4 & 0 & 0.273 & -7.999 & 1.726 \\
\hline 0 & 6 & r & 1.890 & -1.759 & -131 \\
\hline 0 & 8 & $\vdots$ & 8.972 & $1 i .705$ & -2.732 \\
\hline 0 & 10 & 8 & 0.971 & 7.702 & -.731 \\
\hline 0 & 12 & $\therefore$ & 5.144 & 5.092 & .052 \\
\hline 0 & 14 & $n$ & 2.616 & -2.501 & -.115 \\
\hline 0 & 16 & it & .793 & -.335 & -458 \\
\hline 0 & 18 & 0 & 3.508 & $\simeq 529$ & -.021 \\
\hline 0 & 20 & 0 & 4.439 & 4.510 & -.070 \\
\hline 0 & 22 & $n$ & $2 \cdot 16:$ & 1.097 & 1.063 \\
\hline 1 & 1 & $\tilde{v}$ & 1.899 & -1.969 & .072 \\
\hline 1 & 3 & 0 & 2.305 & -2.100 & -.203 \\
\hline 1 & 5 & 0 & 2.760 & -2.860 & .100 \\
\hline 1 & 7 & 0 & 4.622 & -5.109 & .487 \\
\hline 1 & 9 & 3 & 3.436 & $2.8 \mathrm{co}$ & .236 \\
\hline 1 & 11 & 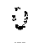 & 2.593 & -2.367 & -.285 \\
\hline 1 & 15 & 0 & .688 & -.800 & .111 \\
\hline 1 & 15 & $\vdots$ & 1.388 & -1.961 & .073 \\
\hline 1 & 17 & 0 & .678 & .722 & -.044 \\
\hline$y$ & 19 & 0 & .311 & -409 & .098 \\
\hline 1 & 21 & 3 & .00 & -.449 & .449 \\
\hline 2 & d) & 0 & $6.1 \times 3$ & -10.510 & 4.415 \\
\hline 2 & 2 & 0 & .831 & $-25 J$ & -.581 \\
\hline 2 & 4 & 9 & 6.144 & 8.128 & -1.985 \\
\hline 2 & 6 & $\therefore$ & 4.440 & 4.096 & -.056 \\
\hline 2 & 8 & 5 & 2.552 & -1.986 & -.565 \\
\hline 2 & 10 & 0 & 4.774 & -5.043 & .27 ? \\
\hline 2 & 12 & 0 & 1.202 & -625 & -.578 \\
\hline 2 & 14 & 0 & 3.525 & 3.408 & .117 \\
\hline 2 & 16 & c & 2.142 & 2.404 & -.262 \\
\hline 2 & 18 & 0 & 1.078 & -1.012 & -.066 \\
\hline 2 & 20 & 0 & 2.758 & -2.98 &.$\lcm{22}$ \\
\hline 3 & 1 & 3 & .430 & -.277 & -.153 \\
\hline 3 & 3 & $n$ & 4.139 & $-4 \cdot 304$ & .165 \\
\hline 3 & 5 & $c$ & 1.333 & $1 \cdot 287$ & .046 \\
\hline 3 & 7 & 0 & .574 & -518 & -.456 \\
\hline 3 & 9 & 0 & .836 & -.813 & -.024 \\
\hline 3 & 11 & 0 & 2.656 & -2.488 & -.168 \\
\hline 5 & 13 & 3 & 2.550 & 2.177 & .174 \\
\hline 3 & 15 & 0 & 1.613 & -1.316 & -.297 \\
\hline 3 & 17 & r & .30 & -.198 & .138 \\
\hline 3 & 19 & 3 & .326 & .425 & -.100 \\
\hline 3 & 21 & $n$ & .30 & .366 & -.356 \\
\hline 4 & 3 & [ & 4.122 & 4.575 & -.453 \\
\hline 4 & 2 & 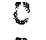 & 1.886 & 1.643 & .242 \\
\hline 4 & 4 & 0 & 1.291 & $-1 \cdot 066$ & -.225 \\
\hline 4 & 6 & 3 & 5.565 & -5.818 & -253 \\
\hline 4 & 8 & 0 & 3.547 & 3.391 & .157 \\
\hline 4 & 10 & 0 & 4.239 & 4.249 & -.010 \\
\hline 4 & 12 & 3 & 1.653 & 1.575 & .78 \\
\hline 4 & 14 & $n$ & 3.565 & -3.516 & -.049 \\
\hline 4 & 16 & 0 & 1.190 & -1.110 & -.080 \\
\hline 4 & 18 & 0 & 1.490 & 1.631 & -.141 \\
\hline 4 & 23 & 3 & 1.904 & 1.876 & .028 \\
\hline 5 & 1 & 0 & 1.225 & -1.171 & -.354 \\
\hline 5 & 3 & 0 & 1.166 & -.907 & -.259 \\
\hline 5 & 5 & c & .569 & -.423 & -148 \\
\hline
\end{tabular}




\begin{tabular}{|c|c|c|c|c|c|}
\hline 5 & 7 & 2 & 2.170 & -7.793 & -.372 \\
\hline 5 & 9 & 0 & 1.364 & 1.112 & .253 \\
\hline 5 & 11 & 0 & 2.089 & -1.890 & -.190 \\
\hline 5 & $1 \leq$ & 4 & .224 & -183 & -842 \\
\hline 5 & 15 & 2 & - voo & -.563 & .363 \\
\hline s & 17 & n & .360 & .592 & -.032 \\
\hline 5 & 19 & 0 & .311 & $-.61 d$ & .36 \\
\hline 6 & 0 & $n$ & 3.649 & -652 & .692 \\
\hline 6 & 2 & 0 & 4.553 & -4.906 & .353 \\
\hline 6 & 4 & 3 & 1.697 & -1.263 & -434 \\
\hline 6 & 8 & 0 & .259 & -.104 & -.155 \\
\hline 6 & 8 & 0 & 3.226 & -2.896 & -.330 \\
\hline 6 & 10 & 0 & 5.613 & -5.534 & -.279 \\
\hline 6 & 12 & 0 & 2.542 & -2.431 & -.111 \\
\hline 6 & 14 & 9 & $.0 \pi i$ & -.135 & .135 \\
\hline 6 & 16 & c & .600 & -1.135 & 1.135 \\
\hline 6 & 18 & 0 & 1.384 & -1.414 & .629 \\
\hline 6 & 20 & 0 & 2.518 & -2.454 & $-\therefore 64$ \\
\hline 7 & 1 & 8 & 1.726 & 1.718 & 50? \\
\hline 7 & 3 & 0 & .810 & .671 & .139 \\
\hline 7 & 5 & 0 & .400 & $.18 \%$ & $-18 r_{3}$ \\
\hline 7 & 7 & 0 & $\operatorname{coc}$ & .064 & -.064 \\
\hline 7 & 9 & 0 & 2.910 & 2.78 .3 & $.13^{m}$ \\
\hline 7 & 11 & 0 & .532 & .395 & .107 \\
\hline 7 & 13 & $n$ & .262 & .331 & -.269 \\
\hline 7 & 15 & 0 & $.50 f^{3}$ & .004 & -.064 \\
\hline 7 & 17 & 0 & .572 & .880 & -.368 \\
\hline 7 & 19 & 0 & .946 & .869 & .077 \\
\hline 8 & 0 & 0 & 3.986 & $\$ .627$ & $-35 z$ \\
\hline 8 & 2 & 0 & .372 & .257 & .115 \\
\hline 8 & 4 & $c$ & .565 & -.396 & -.169 \\
\hline 8 & 6 & 0 & 2.489 & -2.051 & -.437 \\
\hline 8 & 8 & 0 & 2.613 & 1.794 & .219 \\
\hline 8 & 10 & 0 & 1.562 & 1.475 & .287 \\
\hline 8 & 12 & 0 & .442 & .413 & $.63 n$ \\
\hline 8 & 14 & 0 & 1.160 & -.976 & -.84 \\
\hline 8 & 16 & $n$ & .213 & -502 & .288 \\
\hline 8 & 18 & 0 & .906 & 1.003 & -.098 \\
\hline 9 & 1 & 0 & 1.407 & 1.419 & -.412 \\
\hline 9 & 3 & 0 & 1.325 & -1.287 & -.238 \\
\hline 9 & 5 & 0 & 1.319 & -1.246 & $-.37^{\overline{3}}$ \\
\hline 9 & 7 & 0 & .642 & -.061 & .019 \\
\hline 9 & 8 & 8 & .892 & .924 & -830 \\
\hline 9 & 11 & 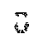 & .605 & .327 & -.027 \\
\hline 9 & 13 & 0 & .386 & -.574 & .188 \\
\hline 9 & 15 & 3 & .413 & -.676 & $.26 ?$ \\
\hline 9 & 18 & 0 & .000 & .217 &.- .17 \\
\hline 10 & 0 & 0 & 3.342 & 2.978 & .64 \\
\hline 10 & 2 & $?$ & .745 & .707 & $\therefore 36$ \\
\hline 10 & 4 & 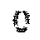 & .445 & -381 & -.064 \\
\hline 10 & 6 & $\mathrm{G}$ & .448 & $-.36:$ & -.368 \\
\hline 10 & 8 & 0 & 1.662 & 1.590 & .087 \\
\hline 10 & 10 & 3 & .838 & .895 & -.56 \\
\hline 10 & 12 & n & -428 & -.291 & -.127 \\
\hline 10 & 14 & is & $\therefore r_{3}$ & .227 & -.227 \\
\hline 10 & 16 & 3 & -463 & -.464 & .631 \\
\hline 11 & 1 & ') & .459 & -.153 & -.286 \\
\hline 17 & $\frac{3}{3}$ & 0 & 1.218 & 1.348 & $-\therefore 3$ \\
\hline 11 & 5 & $r$ & 2.039 & 1.989 & .100 \\
\hline 11 & 7 & 0 & .457 & $.40:$ & .57 \\
\hline 11 & 9 & 0 & .234 & .130 & .104 \\
\hline 11 & 11 & 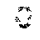 & .491 & .411 & .481 \\
\hline
\end{tabular}




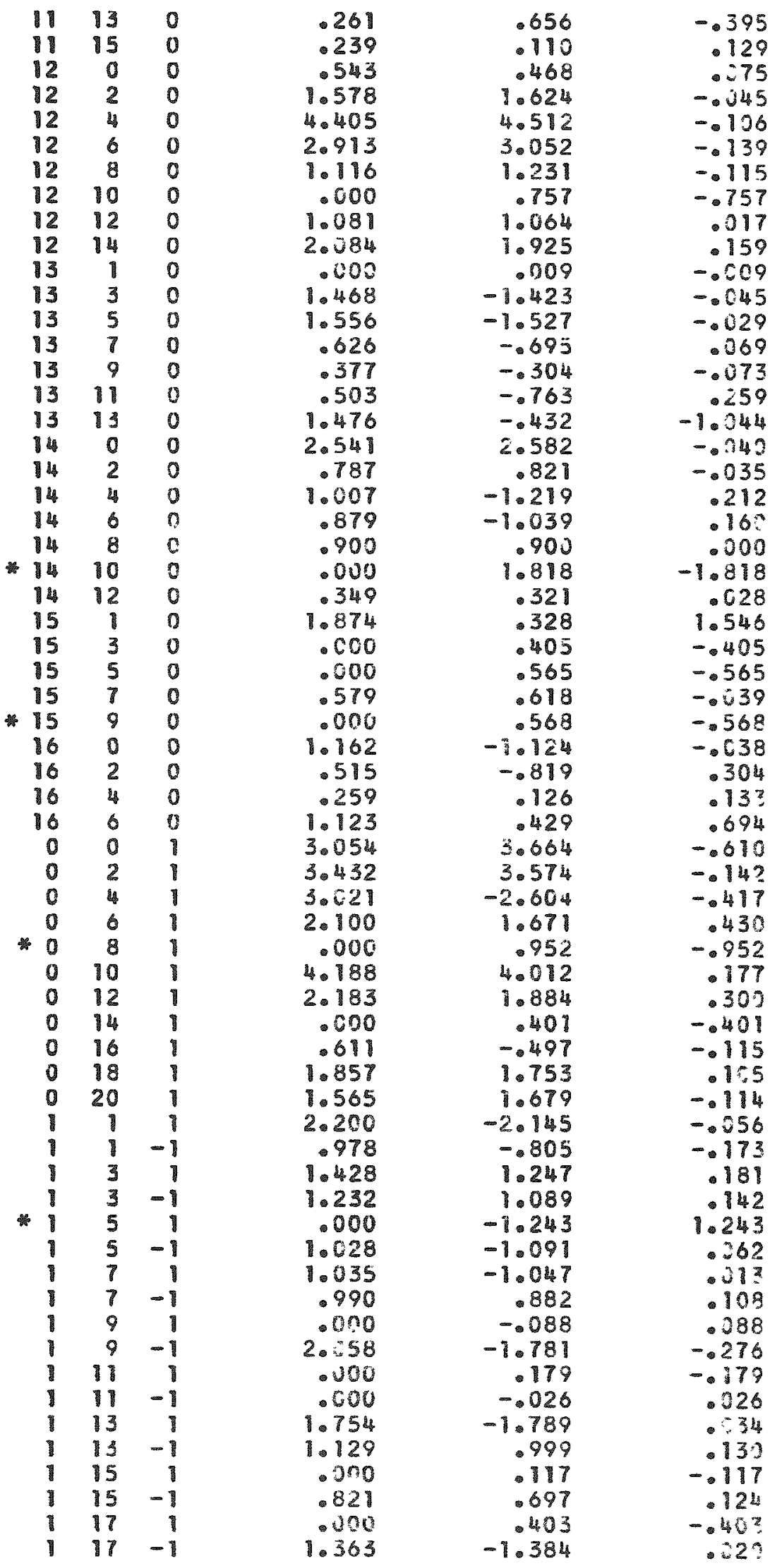




\begin{tabular}{|c|c|c|c|c|c|}
\hline 1 & 19 & 1 & .000 & -.264 & $-26^{4}$ \\
\hline 1 & 19 & -1 & .545 & -.491 & -.253 \\
\hline 1 & 21 & 1 & .050 & -.608 & .608 \\
\hline 1 & 21 & -1 & .020 & .494 & -.494 \\
\hline 2 & 0 & 1 & 4.977 & -5.842 & $.86 \mathrm{~A}$ \\
\hline 2 & 0 & -1 & 2.886 & -3.036 & .15. \\
\hline 2 & 2 & 1 & .691 & -.280 & -.42 \\
\hline 2 & 2 & -1 & 3.769 & $-5.802^{\prime}$ & .34 \\
\hline 2 & 4 & 1 & 3.234 & 2.754 & .481 \\
\hline 2 & 4 & -1 & 3.101 & -2.939 & -162 \\
\hline 2 & 6 & 1 & 4.931 & 4.926 & .306 \\
\hline 2 & 6 & -1 & 4.144 & -4.061 & -.684 \\
\hline 2 & 8 & $\mathbb{I}$ & 1.085 & -.578 & -.517 \\
\hline 2 & 8 & -1 & 2.024 & -2.329 & -.295 \\
\hline 2 & 10 & 1 & 4.469 & -4.491 & .022 \\
\hline 2 & 10 & -1 & 3.451 & -3.203 & -.248 \\
\hline 2 & 12 & 1 & .923 & -.552 & -.371 \\
\hline 2 & 12 & -1 & 2.382 & -2.104 & -.278 \\
\hline 2 & 14 & 1 & 3.688 & 3.415 & .273 \\
\hline 2 & 14 & -1 & 2.059 & -1.877 & -.182 \\
\hline 2 & 16 & 1 & 3.063 & 2.434 & .629 \\
\hline 2 & 16 & -1 & 1.296 & -1.166 & $-.34 n$ \\
\hline 2 & 18 & 7 & 1.796 & -1.045 & -.261 \\
\hline 2 & 18 & -1 & 1.875 & -1.950 & .075 \\
\hline 2 & 20 & 1 & 1.139 & -.980 & -.159 \\
\hline 2 & 20 & -1 & 1.442 & -1.369 & -.073 \\
\hline 2 & 22 & -1 & .30 & -.949 & .949 \\
\hline 3 & 1 & 1 & 1.011 & $-.88 j$ & -131 \\
\hline 3 & 1 & -1 & 1.434 & 1.224 & .210 \\
\hline 3 & 3 & 1 & .736 & -.684 &.- .352 \\
\hline 3 & 3 & -1 & 1.652 & 1.579 & .273 \\
\hline 3 & 5 & 1 & 1.900 & 1.801 & .299 \\
\hline 3 & 5 & -1 & 1.590 & -1.159 & $-.23 ?$ \\
\hline 3 & 7 & 1 & .674 & .554 & $.25 \%$ \\
\hline 3 & 7 & -1 & 1.729 & 1.481 & .24 .3 \\
\hline 3 & 9 & 1 & 2.944 & -2.668 & -.276 \\
\hline 4 & 9 & -1 & .793 & .702 & .092 \\
\hline 3 & 11 & 1 & .586 & .480 & .197 \\
\hline 3 & 11 & -1 & 2.103 & 2.025 & $.08^{n}$ \\
\hline 3 & 13 & 1 & 1.475 & 1.337 & .139 \\
\hline 3 & 15 & -1 & 1.089 & -1.073 & -.016 \\
\hline s & 15 & 1 & .300 & -113 & .113 \\
\hline 3 & 15 & -1 &. $\cos$ & .083 & $-\therefore 80$ \\
\hline 3 & 17 & 1 & .636 & -.918 & .253 \\
\hline 3 & 17 & -1 & 1.258 & 1.209 & -.201 \\
\hline 3 & 19 & 1 & 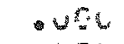 & .374 & -.374 \\
\hline 3 & 19 & -1 & .000 & .453 & -.453 \\
\hline 3 & 21 & 1 & .400 & .116 & -.116 \\
\hline 3 & 21 & -1 & .200 & -.091 & .391 \\
\hline 4 & 3 & 1 & $1.64 \mathrm{~L}$ & -1.254 & -.386 \\
\hline 4 & 0 & -1 & 3.607 & -3.756 & .149 \\
\hline 4 & 2 & 1 & $5.4: 1$ & 5.614 & -212 \\
\hline 4 & 2 & -1 & 1.35 .8 & -1.36 .9 & -.499 \\
\hline 4 & 4 & 1 & .451 & .468 & -.017 \\
\hline 4 & 4 & -1 & .887 & .604 & .283 \\
\hline 4 & 6 & 1 & 1.242 & -1.177 & -.065 \\
\hline 4 & 6 & -1 & .785 & .751 & .034 \\
\hline 4 & 8 & 1 & .549 & -.276 & -.277 \\
\hline 4 & 8 & -1 & 1.620 & -1.187 & $-43 z$ \\
\hline 4 & 10 & 1 & 6.549 & 6.914 & -.364 \\
\hline 4 & 10 & -1 & 2.753 & -2.482 & -.271 \\
\hline 4 & 12 & 1 & 2.633 & 2.554 & .279 \\
\hline
\end{tabular}




\begin{tabular}{|c|c|c|c|c|c|}
\hline & & & & & \\
\hline 4 & 12 & -1 & 1.966 & -1.714 & -.252 \\
\hline 4 & 14 & 1 & 1. 014 & -1.067 & .353 \\
\hline 4 & 14 & -1 & 1.88 .4 & 1.183 & .112 \\
\hline 4 & 16 & 1 & .000 & -.228 & .228 \\
\hline 4 & 16 & -1 & .479 & -.362 & -117 \\
\hline 4 & 18 & $y$ & 1.564 & 1.683 & -.118 \\
\hline 4 & 18 & -1 & .532 & -.520 & -.311 \\
\hline 4 & 20 & 1 & 2.380 & 2.482 & -.102 \\
\hline 4 & 20 & -1 & .000 & -.756 & .756 \\
\hline 5 & 1 & 1 & 1.226 & -1.190 & -.336 \\
\hline * & 1 & -1 & .000 & -2.156 & 2.156 \\
\hline 5 & 3 & 1 & .644 & -.632 & -.12 \\
\hline 蔽 & 3 & -1 & .900 & 2.209 & -2.209 \\
\hline & 5 & 1 & .892 & .764 & -338 \\
\hline 䌿 & 5 & -1 & .000 & 2.477 & -2.477 \\
\hline 5 & 7 & 1 & 1.286 & 1.222 & .363 \\
\hline 卷 & 7 & -1 & .000 & 1.384 & -1.384 \\
\hline & 9 & 1 & 1.988 & -1.777 & -.213 \\
\hline * & 8 & -1 & .000 & -3.644 & 3.644 \\
\hline 5 & 11 & 1 & 2.315 & $-2 \cdot 172$ & -.142 \\
\hline 类 & 11 & -1 & .000 & .254 & -254 \\
\hline 5 & 13 & 1 & .634 & .583 & .354 \\
\hline 费 & 13 & -1 & .000 & 2.068 & -2.568 \\
\hline 5 & 15 & 1 & 1.378 & 1.454 & -.376 \\
\hline 费 & 15 & -1 & .000 & .357 & -.357 \\
\hline 5 & 17 & 1 & 1.147 & $-1 \cdot 123$ & -.024 \\
\hline * & 17 & -1 & .00 & -1.009 & 1.009 \\
\hline & 19 & 1 & .000 & -680 & .680 \\
\hline \# & 18 & -1 & .000 & -.601 & .601 \\
\hline * & 21 & -1 & .000 & .569 & -.569 \\
\hline 6 & 0 & 1 & 5.759 & -6.272 & .514 \\
\hline 6 & 0 & -1 & 2.112 & -2.063 & -.049 \\
\hline 6 & 2 & 1 & 3.821 & $=3.757$ & -.064 \\
\hline * & 2 & -1 & .000 & -1.58. & 1.580 \\
\hline & 4 & 1 & 2.617 & -2.079 & -.538 \\
\hline * & 4 & -1 & .000 & 1.997 & -1.997 \\
\hline & 6 & 1 & 2.775 & 2.363 & .412 \\
\hline 햏 & 6 & -1 & .004 & -4.021 & 4.021 \\
\hline 6 & 8 & I & 4.084 & -3.885 & -.199 \\
\hline * & 8 & -1 & .000 & $-2 \cdot 362$ & 2.362 \\
\hline 6 & 10 & 1 & 5.371 & -5.926 & - 35 \\
\hline * & 10 & -1 & $.00 \mathrm{C}$ & .566 & -.566 \\
\hline 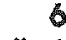 & 12 & 1 & 1.928 & -1.819 & -109 \\
\hline 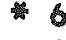 & 12 & -1 & .000 & .411 & -.411 \\
\hline 6 & 14 & 1 & .766 & .609 & .157 \\
\hline 党 & 14 & -1 & .000 & -2.622 & 2.622 \\
\hline 6 & 16 & 1 & .564 & .689 & -.125 \\
\hline * & 16 & -1 & .000 & -1.793 & 1.792 \\
\hline 6 & 18 & $y$ & 1.901 & -1.865 & -.038 \\
\hline * & 18 & -1 & $.20 c$ & -.702 & .702 \\
\hline 8 & 20 & -1 & .200 & -.067 & .267 \\
\hline 7 & 1 & 1 & 2.957 & 2.965 & -.328 \\
\hline * & 1 & -1 & .500 & .984 & -.984 \\
\hline 7 & 3 & 1 & 2.251 & 2.014 & .237 \\
\hline 常 & 3 & -1 & .078 & 1.064 & -1.264 \\
\hline 7 & 5 & 1 & 1.666 & -1.563 & -.113 \\
\hline 范 & 5 & -1 & .025 & .255 & -.255 \\
\hline 7 & 7 & 1 & .510 & $-.43 v$ & $-\therefore 82$ \\
\hline * & 7 & -1 & .090 & $\therefore 091$ & -2.891 \\
\hline 7 & 9 & 1 & $3.97:$ & 5.929 & .047 \\
\hline * & 9 & -1 & .570 & .503 & -.503 \\
\hline 7 & 11 & 1 & 2.618 & 2.849 & -.231 \\
\hline
\end{tabular}




\begin{tabular}{|c|c|c|c|c|c|}
\hline 7 & 11 & -1 & .000 & -.974 & .974 \\
\hline 7 & 13 & 1 & 1.367 & -1.371 & .004 \\
\hline * & 13 & $-i$ & .000 & .678 & \\
\hline 7 & 15 & $i$ & .000 & .077 & $-\div 7$ \\
\hline * & 15 & -1 & - $3 n$ & 1.692 & -1.69 \\
\hline 7 & 17 & 1 & .771 & .747 & .02 \\
\hline \% & 17 & -1 & .000 & .634 & -.63 \\
\hline 7 & 19 & 1 & $.0 \% 5$ & 1.028 & -1.02 \\
\hline * & 19 & -1 & .000 & -.219 & .23 \\
\hline 8 & 0 & 1 & 1.035 & 1.075 & -.03 \\
\hline 8 & 0 & -1 & 3.685 & 3.381 & .30 \\
\hline 8 & 2 & 1 & -436 & $-.01:$ & .01 \\
\hline$* 8$ & 2 & -1 & .000 & 1.937 & -1.95 \\
\hline 8 & 4 & 1 & 6.393 & -6.571 & .17 \\
\hline 费 & 4 & -1 & .070 & 4.169 & $-4 \cdot 16$ \\
\hline 8 & 6 & 1 & 3.446 & $-\pi^{\pi} .324$ & -.12 \\
\hline 尊 & 6 & -1 & .000 & 6.115 & -6.11 \\
\hline 8 & 8 & I & .533 & -.451 & -.88 \\
\hline * & 8 & -1 & .030 & 2.723 & -2.72 \\
\hline 8 & 10 & 1 & 1.345 & 1.030 & .30 \\
\hline * & 10 & -1 & ono & -.664 & .56 \\
\hline 8 & 12 & 1 & .726 & -.505 & -.22 \\
\hline * & 12 & -1 & - U. & 1.311 & -1.3 \\
\hline 8 & 14 & 1 & 1.848 & -1.836 & $-.0 \mathrm{i}$ \\
\hline * & 14 & -1 & .000 & 3.587 & $-3 \cdot 58$ \\
\hline 8 & 16 & $I$ & 1.961 & $-i=116$ & 07 \\
\hline * & 16 & -1 & .000 & 2.216 & -2.2 \\
\hline 8 & 8 & 1 & .000 & -.314 & - 3 \\
\hline * & 18 & -1 & .000 & 1.337 & -1.3 \\
\hline 9 & 1 & 1 & 1.868 & -3.706 & -1 \\
\hline$\%$ & 1 & -1 & .000 & -.367 & .36 \\
\hline 9 & 3 & 1 & 1.767 & 1.505 & .26 \\
\hline * & 3 & -1 & .000 & -.108 & .1 \\
\hline 9 & 5 & I & 3.947 & 3.901 & \\
\hline \# & 5 & -1 & .000 & -3.076 & 3.37 \\
\hline 9 & 7 & 1 & .779 & .742 & .03 \\
\hline * & 7 & -1 & .000 & -2.756 & 2.75 \\
\hline 9 & 9 & 1 & 3.089 & -2.951 & -.1 \\
\hline 要 & 9 & -1 & .000 & .902 & -.93 \\
\hline 9 & 11 & 1 & .020 & -.144 & .11 \\
\hline * & 11 & -1 & - unc & .964 & -.9 \\
\hline 9 & 13 & 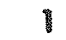 & 1.366 & 1.381 & -.8 \\
\hline * & 13 & -1 & .000 & -1.856 & 1.85 \\
\hline 9 & 15 & 1 & .910 & .962 & -.5 \\
\hline 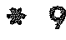 & 15 & -1 & .000 & -1.799 & 1.7 \\
\hline 9 & 17 & 1 & .300 & -.668 & .66 \\
\hline *9 & 17 & -1 & .000 & .052 & -.03 \\
\hline * 9 & 19 & -1 & .000 & -.575 & \\
\hline 10 & 0 & 1 & 0.967 & -7.615 & \\
\hline 10 & 0 & -1 & 5.968 & 6.416 & -.4 \\
\hline 10 & 2 & 1 & 2.779 & -2.549 & -.2 \\
\hline 10 & 2 & -1 & 2.803 & 2.727 & \\
\hline 10 & 4 & 1 & 1. 135 & .929 & .2 \\
\hline 10 & 4 & -1 & .720 & -.296 & -24 \\
\hline 10 & 6 & 1 & .000 & -.288 & .28 \\
\hline 10 & 8 & -1 & 1.228 & -.925 & -30 \\
\hline 10 & 8 & 1 & 3.702 & -3.558 & -.14 \\
\hline 10 & 8 & -1 & 3.549 & 3.176 & .37 \\
\hline 10 & 10 & $I$ & 3.423 & -3.448 & $x^{2}$ \\
\hline 10 & 10 & -1 & 3.157 & 2.881 & .2 \\
\hline 10 & 12 & 1 & 2.041 & -3.987 & -.8 \\
\hline 10 & 12 & -1 & 1.741 & 1.706 & \\
\hline
\end{tabular}




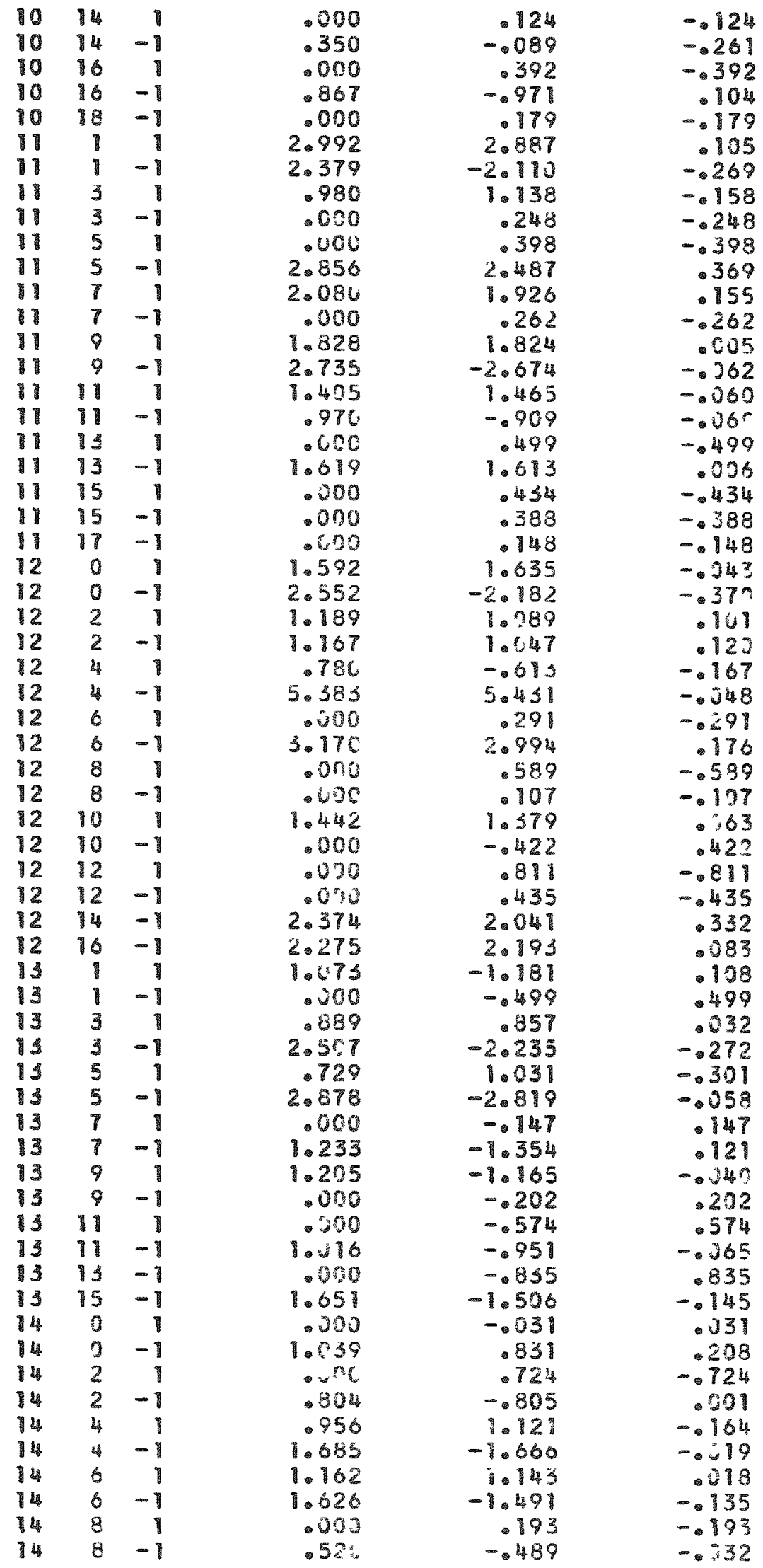




\begin{tabular}{|c|c|c|c|c|c|}
\hline 14 & 10 & -1 & .000 & .004 & -.034 \\
\hline 14 & 12 & -1 & .000 & .036 & -.036 \\
\hline 15 & 1 & 1 & .000 & 1.132 & -1.132 \\
\hline 15 & 1 & -1 & 1.474 & -1.529 & .255 \\
\hline 15 & 3 & 1 & .000 & .324 & -.324 \\
\hline 15 & 3 & -1 & 1.293 & 1.203 & .099 \\
\hline 15 & 5 & 1 & -40 & .054 & -.054 \\
\hline 15 & 5 & -1 & 2.195 & 2.088 & .027 \\
\hline 15 & 7 & -1 & .000 & .198 & -.198 \\
\hline 15 & 9 & -1 & .662 & -.775 & .113 \\
\hline 15 & 11 & -1 & .000 & -.538 & .538 \\
\hline 16 & 0 & -1 & 1. 323 & -1.446 & .123 \\
\hline 16 & 2 & -1 & .970 & -.958 & -.011 \\
\hline 16 & 4 & -1 & DCU & $16^{4}$ & -.164 \\
\hline 16 & 6 & -1 & $.00 u$ & .063 & -.063 \\
\hline 16 & 8 & -1 & .000 & -.701 & .701 \\
\hline 17 & 1 & -1 & .800 & .579 & -.579 \\
\hline 17 & 3 & -1 & 1.160 & -1.205 & .045 \\
\hline 17 & 5 & -1 & 1.697 & -1.627 & -.071 \\
\hline 0 & 0 & 2 & 4.632 & -5.322 & .720 \\
\hline 0 & 2 & 2 & 3.450 & - \pm 205 & -.245 \\
\hline 0 & 4 & 2 & 3.273 & 2.626 & .447 \\
\hline 0 & 6 & 2 & .00 & 1.331 & $-1.33^{\circ}$ \\
\hline 0 & 8 & 2 & 3.088 & $-c .741$ & -.347 \\
\hline 0 & 10 & 2 & 3.159 & -2.769 & -.373 \\
\hline 0 & 12 & 2 & 1.558 &.- .55 & -.043 \\
\hline 0 & 14 & 2 & .674 & .714 & -.349 \\
\hline 0 & 16 & 2 & 1.955 & 1.763 & .172 \\
\hline 0 & 18 & 2 & .715 & -.706 & -.029 \\
\hline 0 & 20 & 2 & 1.098 & -1.141 & .042 \\
\hline 1 & 1 & 2 & .732 & -.698 & -.034 \\
\hline 1 & 1 & -2 & .003 & $\therefore 176$ & -3.176 \\
\hline 1 & 3 & 2 & $.48 \mathrm{C}$ & .148 & -32 \\
\hline \% & 3 & -2 & .300 & 2.777 & -2.777 \\
\hline 1 & 5 & 2 & 1.719 & 1.597 & .122 \\
\hline 剙 & 5 & -2 & -36 & -3.105 & 3.105 \\
\hline 1 & 7 & 2 & 3.494 & 3.320 & .174 \\
\hline * & 7 & -2 & .010 & 1.614 & -1.614 \\
\hline 1 & 9 & 2 & 3.696 & -3.617 &.- .84 \\
\hline * & 9 & -2 & $.30:$ & 2.436 & -2.436 \\
\hline 1 & 11 & 2 & .505 & .401 & .324 \\
\hline * & 11 & -2 & .000 & 3.618 & -3.618 \\
\hline 1 & 13 & 2 & $-00^{4} \times x^{4}$ & .174 & -.174 \\
\hline * & 13 & -2 & .000 & $-\therefore 362$ & 2.362 \\
\hline 1 & 15 & 2 & .973 & 1.088 & -.114 \\
\hline * & 15 & -2 & $.30 \mathrm{C}$ & .382 & -.382 \\
\hline 1 & 17 & 2 & .424 & -.749 & .324 \\
\hline * & 17 & -2 & .000 & .406 & -405 \\
\hline I & 19 & 2 & $.3 n 0$ & .167 & -.167 \\
\hline * & 19 & -2 & .00 & .845 & -.343 \\
\hline 1 & 21 & 2 & .040 & .144 & -.144 \\
\hline * & 21 & -2 & .000 & .035 & -.833 \\
\hline 2 & 0 & 2 & 3.609 & -3.376 & -.233 \\
\hline 2 & 0 & -2 & 5.302 & A. 227 & -2.925 \\
\hline 2 & 2 & 2 & .823 & -.734 & -.389 \\
\hline 2 & 2 & -2 & 2.496 & -7.233 & -.263 \\
\hline 2 & 4 & 2 & 1.331 & -1.203 & -.128 \\
\hline 2 & 4 & -2 & 9.850 & -16.404 & 6.546 \\
\hline 2 & 6 & 2 & 2.165 & -1.851 & -.394 \\
\hline 2 & 6 & -2 & 5.5 .3 & -5.797 & .294 \\
\hline 2 & 8 & 2 & 5.010 & -2.73 & -.277 \\
\hline 2 & 8 & -2 & 1.229 & -.450 & -.574 \\
\hline
\end{tabular}




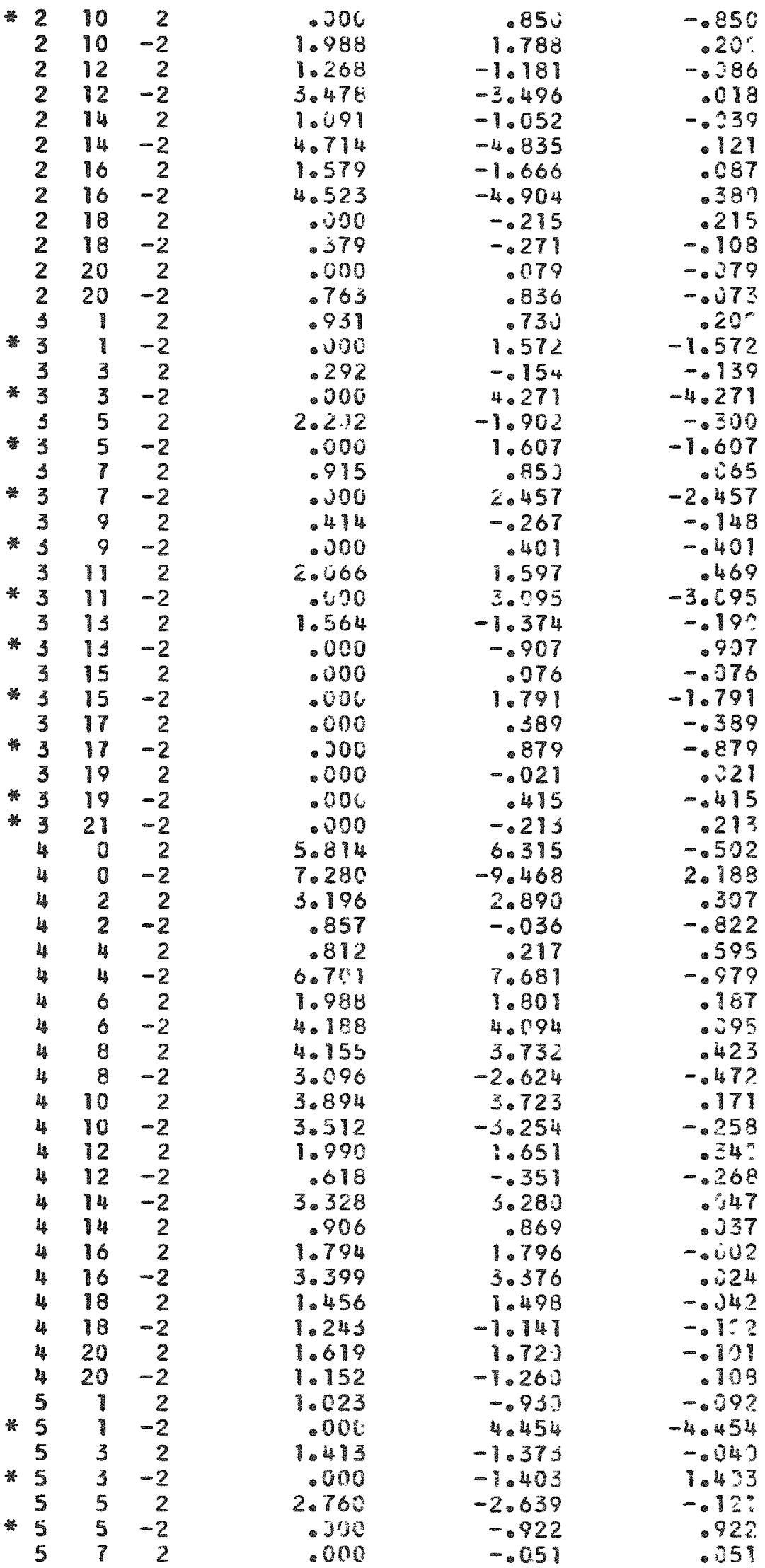




\begin{tabular}{|c|c|c|c|c|c|}
\hline 吗 & 7 & -2 & $\begin{array}{r}.000 \\
-70\end{array}$ & $\begin{array}{r}3.739 \\
-1049\end{array}$ & $\begin{array}{r}-3.739 \\
-\quad 30\end{array}$ \\
\hline * & $\begin{array}{l}9 \\
9\end{array}$ & $\begin{array}{r}2 \\
-2\end{array}$ & $\begin{array}{l}1.679 \\
.978\end{array}$ & $\begin{array}{r}-1.049 \\
-.854\end{array}$ & $\begin{array}{r}-.30 \\
.854\end{array}$ \\
\hline & 11 & 2 & .56 & $\begin{array}{l}-.034 \\
-.590\end{array}$ & $\begin{array}{r}134 \\
-129\end{array}$ \\
\hline$\%$ & 11 & -2 & .000 & 1.972 & -1.972 \\
\hline & 13 & 2 & 1.371 & -1.211 & -.160 \\
\hline * & 13 & -2 & .000 & .309 & -309 \\
\hline & 15 & 2 & .070 & -.541 & .541 \\
\hline * & 15 & -2 & .08 & -.496 & .496 \\
\hline & 17 & 2 & .000 & -.373 & .373 \\
\hline * & 17 & -2 & .000 & -1.21 & $1.21 \%$ \\
\hline & 19 & 2 & - crav & -.085 & $.09^{5}$ \\
\hline 莫 & 19 & -2 & $-6=6$ & 1.283 & $-1.28 ?$ \\
\hline * & 21 & -2 & .500 & $.40 i$ & -400 \\
\hline & 0 & 2 & 2.158 & -1.773 & -.385 \\
\hline & a & -2 & 3.686 & $=847$ & $-16 \%$ \\
\hline & 2 & 2 & .05 & .058 & -058 \\
\hline & 2 & -2 & 4.806 & 4.642 & .167 \\
\hline & 4 & 2 & 1.708 & 1.616 & .592 \\
\hline & 4 & -2 & 5.499 & -3.54 & -41 \\
\hline & 6 & 2 & 2.431 & 2.032 & .569 \\
\hline & 6 & -2 & 3.356 & $-=437$ & $.38 ?$ \\
\hline & 8 & 2 & 1.345 & -1.119 & -.226 \\
\hline & 8 & -2 & 1.73 & .772 & .301 \\
\hline & 10 & 2 & .438 & -.526 & .088 \\
\hline & 10 & -2 & 6.879 & 7.280 & -.421 \\
\hline & 12 & 2 & .60 & .331 & -.331 \\
\hline & 12 & -2 & .000 & .119 & -119 \\
\hline & 14 & 2 & .715 & .740 & -.027 \\
\hline & 14 & -2 & 3.794 & -3.842 & .047 \\
\hline & 16 & 2 & .733 & .829 & -.096 \\
\hline & 16 & -2 & 1.740 & -1.837 & - บ96 \\
\hline & 18 & 2 & .000 & -.098 & .098 \\
\hline & 18 & -2 & 1.520 & 1.589 & - 469 \\
\hline & 20 & -2 & 1.972 & 1.956 & -.054 \\
\hline & 1 & 2 & .911 & .840 & .371 \\
\hline * & 1 & -2 & .000 & $-1.23 \mathrm{~J}$ & 1.230 \\
\hline & 3 & 2 & $.6 n$ & $-25=$ & -253 \\
\hline * & 3 & -2 & .000 & 3.722 & $-3.72 ?$ \\
\hline & 5 & 2 & 2.100 & -1.835 & -.271 \\
\hline * & 5 & -2 & $.0 n 2$ & $2 \cdot 386$ & -2.386 \\
\hline & 7 & 2 & ax & -.106 & .136 \\
\hline * & 7 & -2 & .000 & 1.949 & -1.949 \\
\hline & 9 & 2 & 1.875 & 1.154 & .021 \\
\hline * & 9 & -2 & 180 & -429 & .429 \\
\hline & 11 & 2 & .746 & .853 & -.108 \\
\hline$*$ & 11 & -2 & $\therefore 88$ & -.234 & .234 \\
\hline & 13 & 2 & .631 & -.669 & .038 \\
\hline * & 13 & -2 & - $\omega^{*}$ & -.160 & .106 \\
\hline & is & 2 & -200 & -.034 & .634 \\
\hline * & 15 & -2 & $-\therefore 90$ & 2.442 & -2.442 \\
\hline & 17 & 2 & .000 & .371 & -.371 \\
\hline * & 17 & -2 & 208 & .421 & -421 \\
\hline * & 19 & -2 & ons & -.410 & .46 \\
\hline & 0 & 2 & 4.399 & 4.666 & -.267 \\
\hline & 0 & -2 & 2.317 & -2.196 & -.21 \\
\hline & 2 & 2 & 1.423 & 1.262 & .61 \\
\hline & 2 & -2 & 1.376 & 1.396 & .197 \\
\hline & 4 & 2 & 3.618 & -3.457 & -.161 \\
\hline & 4 & -2 & 5.135 & 5.194 & $-\therefore 6 \%$ \\
\hline & 6 & 2 & 1.315 & -1.445 & \\
\hline & 6 & -2 & 8.186 & 8.932 & -.747 \\
\hline
\end{tabular}




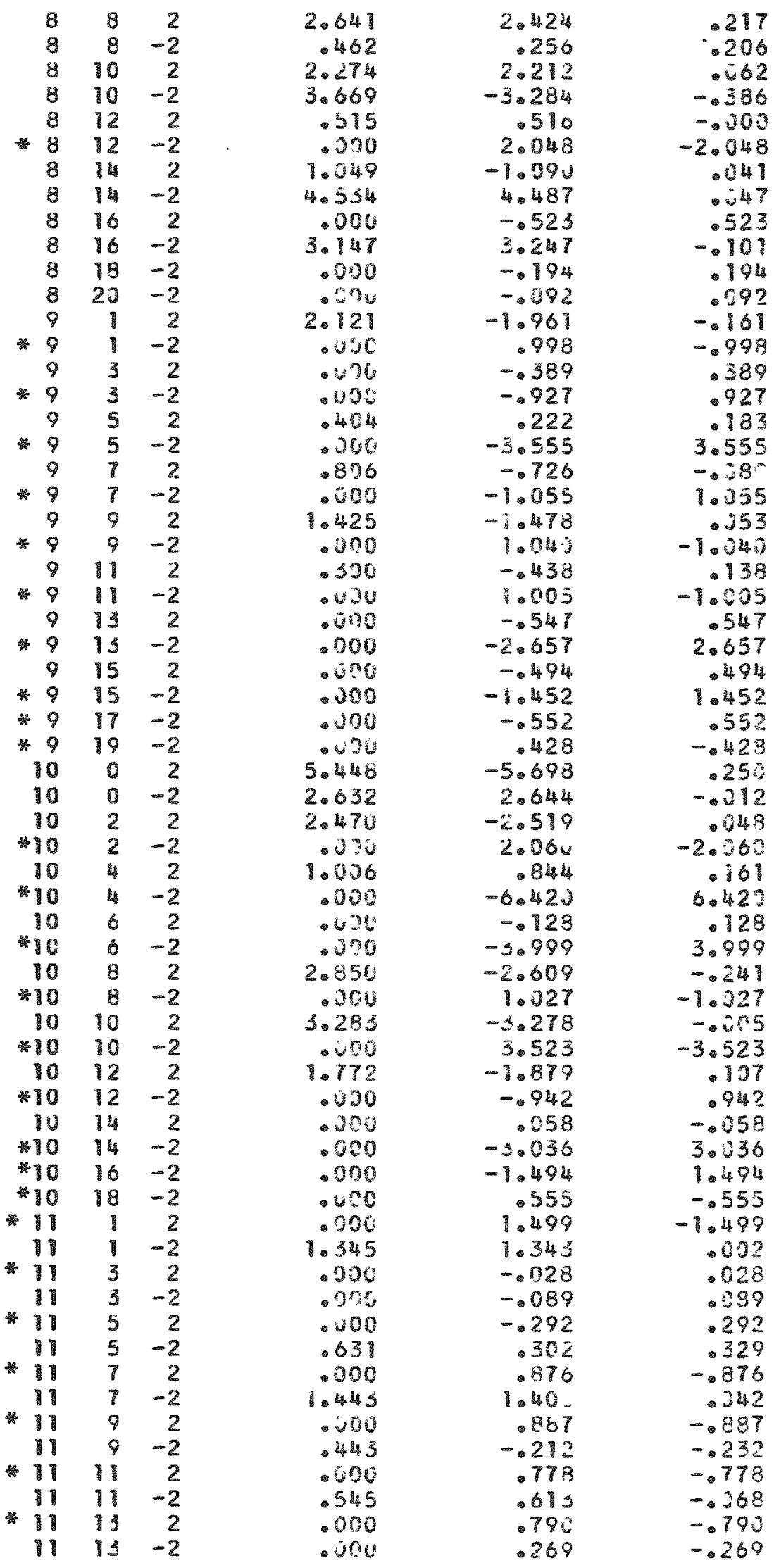




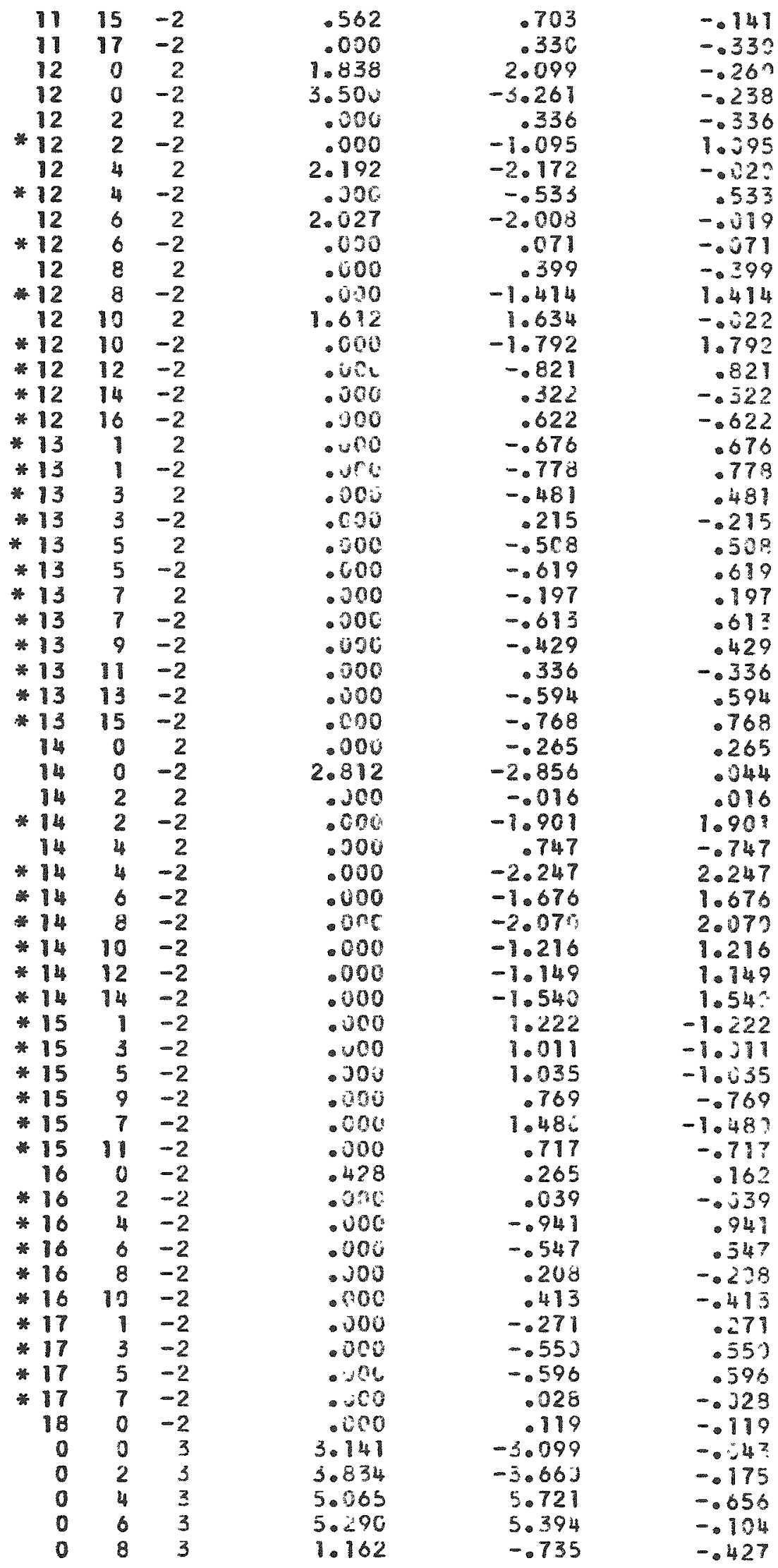




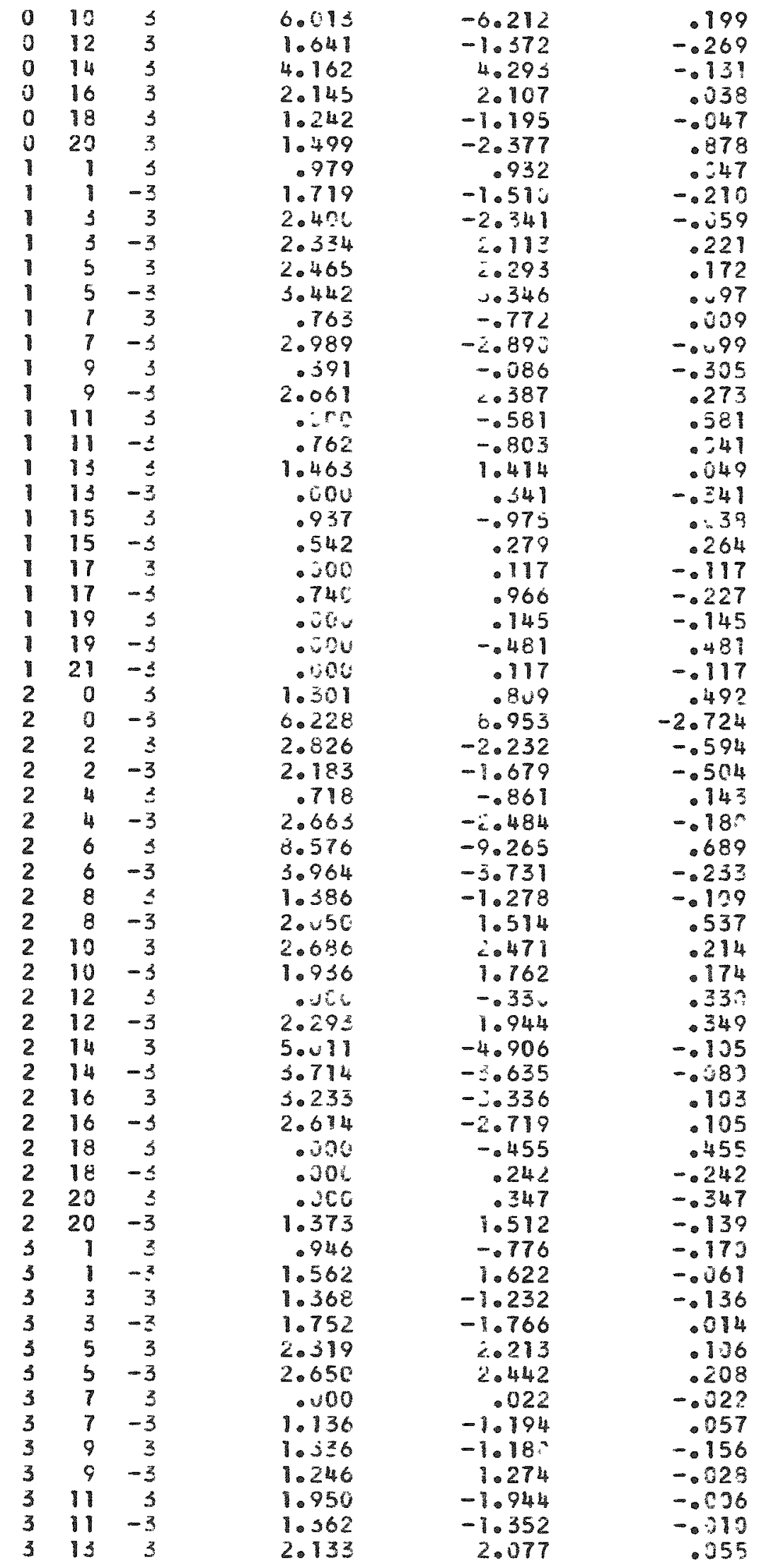




\begin{tabular}{|c|c|c|c|c|c|}
\hline 3 & 13 & -3 & 1.992 & 1.961 & $\therefore 31$ \\
\hline 3 & 15 & -3 & .600 & .115 & -.115 \\
\hline 3 & 37 & 3 & .60 & $-.47 !$ & .475 \\
\hline 3 & 17 & -3 & - $\omega^{n}$ & -.247 & .247 \\
\hline 3 & 19 & 3 & .0 .0 & -.777 & .777 \\
\hline 3 & 19 & -3 & .37 & -.374 & .374 \\
\hline 3 & 21 & -3 & .250 & .434 & -.434 \\
\hline 4 & 0 & 3 & 1.077 & .564 & .442 \\
\hline 4 & 0 & -3 & 2.336 & 2.216 & - \$18 \\
\hline 4 & 2 & 3 & 1.779 & -1.385 & -.394 \\
\hline 4 & 2 & -3 & 3.758 & 3816 & -.257 \\
\hline 4 & 4 & 3 & 5.373 & 5.255 & .125 \\
\hline 4 & 4 & -3 & 6.354 & 7.239 & -.685 \\
\hline 4 & 6 & 3 & $4.77 \%$ & 4.505 & .264 \\
\hline 4 & 6 & -3 & 4.623 & 4.792 & -.169 \\
\hline 4 & 8 & 3 & .669 & .447 & .222 \\
\hline 4 & 8 & -3 & 3.495 & $\approx 232$ & .261 \\
\hline 4 & 10 & 3 & 3.172 & -2.642 & -.527 \\
\hline 4 & 10 & -3 & 2.148 & 1.713 & .435 \\
\hline 4 & 12 & 3 & .858 & .754 & .104 \\
\hline 4 & 12 & -3 & $1 .: 98$ & 1.191 & -.297 \\
\hline 4 & 14 & -3 & 4.140 & 4.194 & -.054 \\
\hline 4 & 14 & 3 & 3.277 & 3.211 & .468 \\
\hline 4 & 16 & 3 & 1.581 & 1.693 & -.112 \\
\hline 4 & 16 & -3 & 3.532 & 3.531 & .002 \\
\hline 4 & 18 & 3 & .50 & .243 & $-.34 z$ \\
\hline 4 & 18 & -3 & .761 & .920 & -139 \\
\hline 4 & 20 & -3 & .396 & -.704 & .754 \\
\hline 5 & 1 & 2 & .839 & -.585 & -.254 \\
\hline 5 & 1 & -3 & 1.153 & 1.086 & .067 \\
\hline 5 & 3 & 3 & 1.751 & -1.616 & -.134 \\
\hline 5 & 3 & -3 & 3.711 & -5.658 & -.53 \\
\hline 5 & 5 & 3 & 3.675 & $-3.53 z$ & -.136 \\
\hline 5 & 5 & -3 & .443 & -211 & -.232 \\
\hline 5 & 7 & 3 & 3.411 & -2.964 & -.237 \\
\hline 5 & 7 & -3 & 2.856 & -2.716 & $-.14 n$ \\
\hline 5 & 9 & 3 & 2.325 & 1.914 & .111 \\
\hline 5 & 9 & -3 & .400 & 1.200 & -1.208 \\
\hline 5 & 11 & 3 & $.20 \%$ & -.614 & .614 \\
\hline 5 & 11 & -3 & .569 & -.780 & .276 \\
\hline 5 & 13 & 5 & 1.245 & $-<.083$ & .838 \\
\hline 5 & 13 & -3 & .760 & -.671 & -.89 \\
\hline 5 & 15 & 3 & 1.190 & -1.464 & .274 \\
\hline 5 & 15 & -3 & $2 . .51$ & $-2.12 s$ & .272 \\
\hline 5 & 17 & 3 & -00 & $.45=$ & -.452 \\
\hline 5 & 17 & -3 & - जot & .324 & -324 \\
\hline 5 & 19 & -3 & .00 & $.18 \%$ & -181 \\
\hline 5 & 21 & -3 & $\because 06$ & -.494 & .494 \\
\hline 6 & 0 & 3 & 6.232 & 6.429 & -197 \\
\hline 6 & 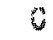 & -3 & $4 \cdot 355$ & $=677$ & .457 \\
\hline 6 & 2 & 3 & .878 & .756 & .115 \\
\hline 6 & 2 & -3 & .517 & .221 & .296 \\
\hline 6 & 4 & 2 & .881 & -.908 & -28 \\
\hline 6 & 4 & -3 & 2.447 & 1.889 & .598 \\
\hline 6 & 6 & 3 & 1.589 & -1.117 & -.252 \\
\hline 6 & 6 & -3 & $.55 t$ & -0.21 & -.215 \\
\hline 6 & 8 & 3 & 3.0 .27 & $\therefore .761$ & .266 \\
\hline 6 & 8 & $-\xi$ & 1.972 & 1.631 & .347 \\
\hline 6 & 10 & $\stackrel{z}{z}$ & 1.977 & .954 & -248 \\
\hline 6 & 10 & -3 & $1 . .73$ & .779 & .295 \\
\hline 6 & 12 & $s$ & $1 . .51$ & $\because 58$ & .091 \\
\hline 6 & 12 & -3 & 1.778 & $\because .57 \mathrm{a}$ & .207 \\
\hline
\end{tabular}




\begin{tabular}{|c|c|c|c|c|c|}
\hline 6 & 14 & 3 & .000 & -.290 & $.29 \%$ \\
\hline 6 & 14 & -5 & .590 & -.414 & -.175 \\
\hline 6 & 16 & 3 & .000 & -.931 & .931 \\
\hline 6 & 16 & -3 & .000 & -.281 & .281 \\
\hline 6 & 18 & -3 & .000 & -.072 & .073 \\
\hline 6 & 20 & -3 & 1. 188 & 1.06 & .328 \\
\hline 7 & 1 & 3 & 2.374 & -2.519 & .145 \\
\hline 7 & 1 & -3 & 3.614 & -3.335 & -.279 \\
\hline 7 & 3 & 3 & .200 & -.145 & $.14 \%$ \\
\hline 7 & 3 & -3 & .911 & .801 & .111 \\
\hline 7 & 5 & 3 & 1.755 & 1.994 & -239 \\
\hline 7 & 5 & -3 & 4.123 & 3.993 & .131 \\
\hline 7 & 7 & 3 & .930 & -1.339 & .178 \\
\hline 7 & 7 & -3 & 2.102 & -7.906 & -.196 \\
\hline$T$ & 9 & $s$ & 1.932 & -2.071 & .139 \\
\hline 7 & 9 & -3 & 2.481 & -2.211 & $-.27 \%$ \\
\hline 7 & 11 & $s$ & .515 & -.702 & .187 \\
\hline 7 & 11 & -5 & 1.835 & -1.789 & -.046 \\
\hline 7 & 13 & 3 & 1.424 & 1.386 & .028 \\
\hline 7 & 13 & -3 & 2.305 & 2.445 & $-.144^{\circ}$ \\
\hline 7 & 15 & 3 & .000 & $.12 v$ & $-12 \%$ \\
\hline 7 & 15 & -3 & .832 & .765 & .367 \\
\hline 7 & 17 & -3 & $1 . \cup 50$ & -1.193 & $.14 ?$ \\
\hline 7 & 19 & -3 & .416 & $-89 z$ & .477 \\
\hline 8 & 0 & 3 & .913 & -.259 & -.654 \\
\hline 8 & 0 & -3 & 2.336 & -2.214 & -.122 \\
\hline 8 & 2 & 3 & 1.734 & $1.54 u$ & .195 \\
\hline 8 & 2 & -3 & .656 & .427 & $.23 \%$ \\
\hline 8 & 4 & 3 & 5.536 & 3.518 & .017 \\
\hline 8 & 4 & -3 & 4.145 & 3.779 & .366 \\
\hline 8 & 6 & 3 & 2.984 & 2.907 & $\therefore 77$ \\
\hline 8 & 6 & -3 & 2.361 & 1.932 & .429 \\
\hline 8 & 8 & 3 & 1.273 & 1.237 & .037 \\
\hline 8 & 8 & -3 & 2.004 & -1.779 & -.225 \\
\hline 8 & 10 & 3 & .698 & .578 & .129 \\
\hline 8 & 10 & -3 & .618 & .559 & .059 \\
\hline 8 & 12 & 3 & 1.549 & 1.406 & .143 \\
\hline 8 & 12 & -3 & 1.694 & 1.533 & $.16^{\circ}$ \\
\hline 8 & 14 & 3 & 2.615 & $\therefore 476$ & .138 \\
\hline 8 & 14 & -3 & 1.179 & 1.103 & .079 \\
\hline 8 & 16 & -3 & .000 & .304 & -.304 \\
\hline 8 & 18 & -3 & .00 & .537 & -537 \\
\hline 8 & 20 & -3 & .000 & -.242 & .242 \\
\hline 9 & 1 & 3 & 1.240 & -.342 & .102 \\
\hline 9 & 1 & -3 & .026 & -.4 .42 & .142 \\
\hline 9 & 3 & 3 & 1.916 & -1.884 & -.332 \\
\hline 9 & 3 & -3 & 3.275 & -2.996 & -.209 \\
\hline 9 & 5 & 3 & 1.179 & -1.179 & .001 \\
\hline 9 & 5 & -3 & 2.213 & $-1.58=$ & $-43^{n}$ \\
\hline 9 & 7 & 3 & .888 & -1.079 & .191 \\
\hline 9 & 7 & -3 & - onc & -.392 & .392 \\
\hline 9 & 9 & 3 & .784 & -847 & .364 \\
\hline 9 & 9 & -3 & .624 & .699 & -.75 \\
\hline 9 & 11 & 3 & 1.235 & $-1 \cdot 223$ & -.015 \\
\hline 9 & 11 & -3 & 2.370 & $-2.43 j$ & .463 \\
\hline 9 & 13 & 3 & .403 & -.488 & -488 \\
\hline 9 & 13 & -3 & 1.579 & -1.507 & -.471 \\
\hline 9 & 15 & -2 & .300 & -.169 & .169 \\
\hline 9 & 17 & -3 & .600 & -.087 & .387 \\
\hline 9 & 19 & -3 & $.00 t$ & -.388 & .388 \\
\hline 10 & 0 & 3 & .352 & .431 & .121 \\
\hline 10 & 0 & -3 & 4.354 & -2.058 & \\
\hline
\end{tabular}




\begin{tabular}{|c|c|c|c|c|c|c|}
\hline & 10 & 2 & 3 & .571 & -.625 & .54 \\
\hline & 10 & 2 & -3 & 4.327 & -152 & -.175 \\
\hline & 10 & 4 & 3 & .000 & -.261 & $26:$ \\
\hline & 10 & 4 & -3 & $3 \cdot 316$ & -3.284 & -.022 \\
\hline & 10 & 6 & 3 & .000 & -.525 & .525 \\
\hline & 10 & 6 & -3 & 1.958 & -1.742 & -.216 \\
\hline & 10 & 8 & 3 & .003 & -.078 & .078 \\
\hline & 10 & 8 & -3 & 1.190 & -1.223 & - $33 ?$ \\
\hline & 10 & 10 & $s$ & - 3บ: & -.184 & .184 \\
\hline & 10 & 10 & -3 & 3.760 & -3.798 & .038 \\
\hline & 10 & 12 & 3 & .000 & -.285 & .285 \\
\hline & 10 & 12 & -3 & 2.358 & -2.465 & .127 \\
\hline & 10 & 14 & -3 & .965 & -.997 & -42 \\
\hline & 10 & 16 & -3 & .663 & -.803 & .741 \\
\hline & 10 & 18 & -3 & 1.472 & -1.007 & -.065 \\
\hline & 11 & 1 & 3 & -200 & .062 & -.062 \\
\hline & 11 & 1 & -3 & .901 & .831 & .070 \\
\hline & 11 & 3 & 3 & .0 .06 & .331 & -.331 \\
\hline & 11 & 3 & -3 & 2.038 & 1.710 & .328 \\
\hline & 11 & 5 & 3 & -6 & .361 & -.361 \\
\hline & 11 & 5 & -3 & 1.141 & $1.13=$ & .008 \\
\hline & 11 & 7 & 3 & - ธot. & -.038 & .038 \\
\hline & 11 & 7 & -3 & .862 & -.817 & $-\infty 45$ \\
\hline & 11 & 8 & 3 & .300 & .289 & -.299 \\
\hline & 11 & 9 & -3 & 1.274 & 1.326 & -.352 \\
\hline & 11 & 11 & -3 & 2.317 & 2.191 & .127 \\
\hline & 11 & 13 & -3 & .628 & .567 & .061 \\
\hline ? & 11 & 15 & -3 & 0.06 & .153 & -.153 \\
\hline & 11 & 17 & -3 & .000 & .557 & -.557 \\
\hline & 12 & 0 & 3 & .000 & -.372 & .372 \\
\hline & 12 & 0 & -3 & $\begin{array}{l}532 \\
-5\end{array}$ & .564 & -.032 \\
\hline & 12 & 2 & $\underline{z}$ & .000 & -.645 & .645 \\
\hline & 12 & 2 & -3 & .652 & -.693 & .038 \\
\hline & 12 & 4 & 3 & .000 & -437 & .437 \\
\hline & 12 & 4 & -3 & 3.228 & -2.176 & -.112 \\
\hline & 12 & 6 & $s$ & .00 & -.781 & .781 \\
\hline & 12 & 6 & -3 & 2.860 & -2.633 & -.227 \\
\hline & 12 & 8 & 3 & .301 & -.003 & .653 \\
\hline & 12 & $\varepsilon$ & -2 & .622 & -.625 & .023 \\
\hline & 12 & 10 & -3 & .732 & .627 & .075 \\
\hline & 12 & 12 & -3 & -20 & -.385 & $\cdot 53^{5}$ \\
\hline & 12 & 14 & -3 & .000 & $-.45=$ & .453 \\
\hline & 12 & 16 & -3 & 1.345 & -1.44. & .176 \\
\hline & 13 & 1 & 3 & .020 & -.734 & .734 \\
\hline & 13 & 1 & -3 & .270 & -.533 & .532 \\
\hline & 13 & 3 & 3 & .06 & -.339 & .339 \\
\hline * & 13 & 3 & -3 & .006 & -.163 & .169 \\
\hline \% & 13 & 5 & -3 & .000 & 1.047 & -1.47 \\
\hline * & 13 & 7 & -3 & 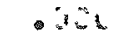 & .424 & -.424 \\
\hline \& & 13 & 9 & -3 & .400 & -1.056 & 1.356 \\
\hline \% & 13 & 11 & -3 & .000 & -487 & .487 \\
\hline * & 13 & 13 & -3 & 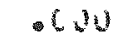 & .62. & $-.62^{\circ}$ \\
\hline * & 13 & 15 & $-\underline{2}$ & .05 & .130 & $-.1 \leq 8$ \\
\hline & 14 & 0 & -3 & 3.734 & $-\ldots .999$ & $\not<65$ \\
\hline & 14 & 2 & -3 & 1.334 & -1.600 & .274 \\
\hline & 14 & 4 & -5 & 1.332 & $3 \cdot 526$ & -.193 \\
\hline & 14 & 6 & -3 & .856 & .144 & .712 \\
\hline & 14 & 8 & -3 & 1.433 &.- .805 & 0.379 \\
\hline & 14 & 10 & $-z$ & 1.415 & -1.557 & .143 \\
\hline & 14 & 12 & -3 & .755 & -.927 & .172 \\
\hline & 14 & 14 & -3 & .253 & .213 & .143 \\
\hline 呟 & 15 & 1 & -3 & .000 & 1.613 & -1.613 \\
\hline
\end{tabular}




\begin{tabular}{|c|c|c|c|c|c|c|}
\hline 券 & 15 & 3 & -3 & -6.80 & .934 & -.934 \\
\hline * & 15 & 5 & -5 & $\therefore 50$ & .485 & -.485 \\
\hline * & 15 & 7 & -3 & .000 & .909 & -929 \\
\hline * & 15 & 9 & -3 & .00 & 1.243 & -1.243 \\
\hline * & 15 & 11 & -3 & .006 & 1.134 & -1.134 \\
\hline * & 15 & 13 & -3 & .300 & 1.005 & -1.635 \\
\hline & 16 & 0 & -3 & 1.699 & 1.690 & .009 \\
\hline & 16 & 2 & -3 & 1.562 & .989 & $\therefore 73$ \\
\hline & 16 & 4 & -3 & .56 & .606 & -.026 \\
\hline & 16 & 6 & -3 & .000 & .477 & -.477 \\
\hline & 16 & 8 & -3 & .000 & .665 & -.665 \\
\hline & 16 & 10 & -3 & 1.366 & 1.317 & .249 \\
\hline & 16 & 12 & -3 & $-2^{2} 00$ & 3.246 & -1.246 \\
\hline & 17 & 1 & -3 & 1.723 & $-2 \cdot 191$ & -468 \\
\hline & 17 & 3 & -5 & 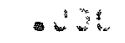 & .04 & $-34^{\circ}$ \\
\hline & 17 & 5 & -3 & .000 & .835 & -.835 \\
\hline & 17 & 7 & -3 & $-\approx 0 y$ & -.494 & .494 \\
\hline & 17 & 9 & -3 & 1.235 & -7.546 & .312 \\
\hline & 18 & 0 & -3 & .512 & -.475 & -.037 \\
\hline * & 18 & 2 & -3 & $.35 \mathrm{C}$ & .051 & -.251 \\
\hline * & 18 & 4 & -3 & .220 & .822 & -.822 \\
\hline & 0 & 0 & 4 & 2.440 & $-3.52 \%$ & -.141 \\
\hline & 0 & 2 & 4 & 1.461 & .953 & .598 \\
\hline & 0 & 4 & 4 & 6.313 & 6.915 & -.603 \\
\hline & 0 & 6 & 4 & 4.172 & $=972$ & .201 \\
\hline & 0 & 8 & 4 & .561 & -.295 & -.267 \\
\hline & 0 & 10 & 4 & 1.450 & -1.26 & $-.19 x$ \\
\hline & 0 & 12 & 4 & .000 & $\angle .295$ & -2.295 \\
\hline & 0 & 14 & 4 & $\operatorname{s.2018}$ & 2.952 & .066 \\
\hline & 0 & 16 & 4 & 1.310 & 1.329 & -.519 \\
\hline & 0 & 18 & 4 & .000 & -.006 & $: 206$ \\
\hline & 1 & 1 & 4 & 1.703 & 1.453 & .251 \\
\hline & 1 & 1 & -4 & .000 & -.314 & 314 \\
\hline & I & 3 & 4 & .430 & .288 & .142 \\
\hline & 1 & 3 & -4 & .000 & -.063 & .063 \\
\hline & 1 & 5 & 4 & 1.522 & 1.374 & .148 \\
\hline & 1 & 5 & -4 & 1.971 & $2 . \cap 12$ &.- .241 \\
\hline & 1 & 7 & 4 & .441 & -.343 & -.097 \\
\hline & 1 & 7 & -4 & .000 & .017 & -.317 \\
\hline & 1 & 9 & 4 & 1.491 & 1.343 & .148 \\
\hline & 1 & 9 & -4 & .511 & .286 & .215 \\
\hline & 1 & 11 & 4 & $.58 b$ & .463 & .128 \\
\hline & 1 & 11 & -4 & 1.0 .14 & -1.509 & -.095 \\
\hline & 1 & 13 & 4 & .406 & .249 & -248 \\
\hline & 1 & 13 & -4 & $1 .: 15$ & .089 & -.386 \\
\hline & 1 & 15 & 4 & .030 & -.099 & 0399 \\
\hline & 1 & 15 & -4 & .000 & .175 & -.175 \\
\hline & 1 & 17 & 4 & 030.2 & .499 & -497 \\
\hline & 1 & 17 & -4 & .000 & -.183 & $.18 \pi$ \\
\hline & 1 & 19 & 4 & $.0 n 0$ & .068 & -.368 \\
\hline & 1 & 19 & -4 & - 186 & .182 & -.192 \\
\hline & 2 & 0 & 4 & 2.797 & 2.705 & .092 \\
\hline & 2 & 0 & -4 & 2.920 & 2.455 & -.514 \\
\hline & 2 & 2 & 4 & .511 & -.333 & -.178 \\
\hline \# & 2 & 2 & -4 & .20 & 1.551 & -1.551 \\
\hline & 2 & 4 & 4 & $6.93 \mathrm{z}$ & -7.411 & .512 \\
\hline * & 2 & 4 & -4 & .500 & .284 & -.284 \\
\hline & 2 & 6 & 4 & 5.827 & -5.871 & .134 \\
\hline * & 2 & 6 & -4 & 00 & .910 & -.916 \\
\hline & 2 & 8 & 4 & .693 & .494 & .189 \\
\hline * & 2 & 8 & -4 & 30 & 1.667 & -1.667 \\
\hline & 2 & 10 & 4 & 2.447 & 2.181 & .265 \\
\hline
\end{tabular}




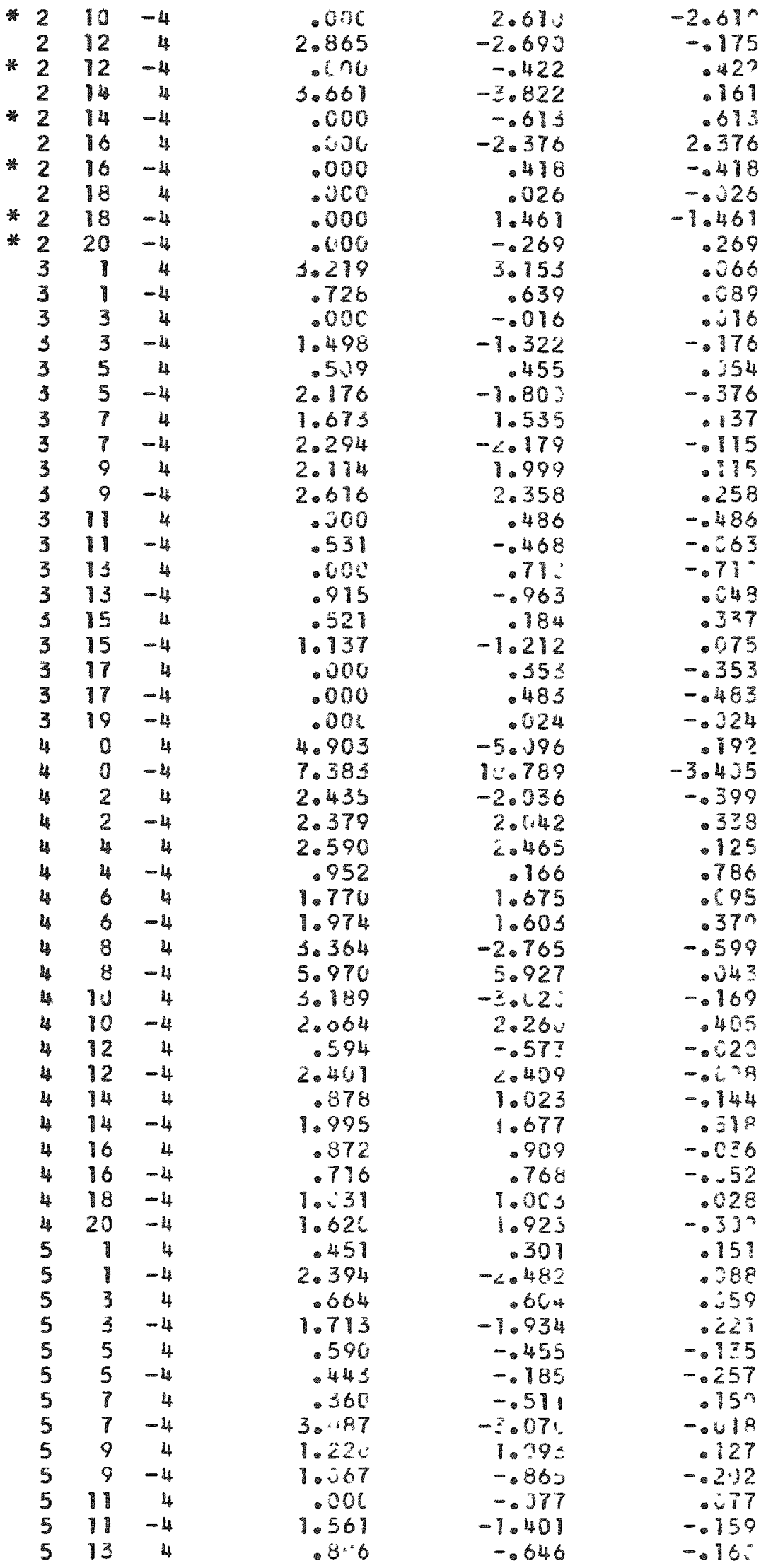




\begin{tabular}{|c|c|c|c|c|c|}
\hline 5 & 13 & -4 & 1.125 & -.996 & -129 \\
\hline 5 & 15 & 4 & -ucu & .115 & -.115 \\
\hline 5 & 15 & -4 & 1.238 & -1.221 & $-\because 17$ \\
\hline 5 & 17 & 4 & .000 &. .574 & .374 \\
\hline 5 & 17 & -4 & .200 & .308 & -.308 \\
\hline 5 & 19 & -4 & .764 & -.737 & -.27 \\
\hline 6 & 3 & 4 & 3.279 & 3.058 & .221 \\
\hline 6 & 0 & -4 & 4.745 & -5.053 & .308 \\
\hline 6 & 2 & 4 & .729 & $-.55 j$ & -.179 \\
\hline 6 & 2 & -4 & 60 & -2.374 & 2.374 \\
\hline 6 & 4 & 4 & 5.437 & -5.667 & .23 \\
\hline * & 4 & -4 & $.00 \mathrm{C}$ & 2.788 & -2.788 \\
\hline 6 & 6 & 4 & 3.127 & -2.981 & -.146 \\
\hline * & 6 & -4 & .020 & -1.249 & 1.249 \\
\hline 6 & 8 & 4 & .620 & .453 & .167 \\
\hline 6 & 8 & -4 & .200 & -2.618 & 2.618 \\
\hline 6 & 10 & 4 & 1.152 & 1.028 & .124 \\
\hline 6 & 10 & -4 & .000 & -1.585 & 1.58 \\
\hline 6 & 12 & 4 & 1.064 & -.823 & -.241 \\
\hline 6 & 12 & -4 & .000 & -2.126 & 2.126 \\
\hline 6 & 14 & 4 & 2.435 & $-2 \cdot 231$ & -174 \\
\hline * 6 & 14 & -4 & $.00 \mathrm{C}$ & .155 & -.155 \\
\hline 6 & 16 & 4 & 2.304 & -2.23 & -.075 \\
\hline 6 & 16 & $-\frac{4}{4}$ & .800 & -.049 & .04 \\
\hline 6 & 18 & -4 & ourb & -.361 & .36 \\
\hline 6 & 20 & -4 & .000 & -2.0113 & 2.01 \\
\hline 7 & 1 & 4 & .237 & .175 & .063 \\
\hline 7 & 1 & -4 & 3.086 & 2.821 & 266 \\
\hline 7 & 3 & 4 & 1.126 & .863 & .16 \\
\hline 7 & 3 & -4 & 4.013 & -3.963 & -.05 \\
\hline 7 & 5 & 4 & 1.286 & 1.281 & .005 \\
\hline 7 & 5 & -4 & 2.738 & -2.373 & -36 \\
\hline 7 & 7 & 4 & .278 & .295 & -.016 \\
\hline 7 & 7 & -4 & .000 & .131 & $-\mid 3$ \\
\hline 7 & 9 & 4 & .000 & .234 & -.23 \\
\hline 7 & 9 & -4 & 1.070 & .930 & .14 \\
\hline 7 & 11 & 4 & .618 & .650 & -.03 \\
\hline 7 & 11 & -4 & .090 & -.281 & .281 \\
\hline 7 & 13 & 4 & .000 & -.083 & .08 \\
\hline 7 & 13 & -4 & 1.093 & 1.189 & -.09 \\
\hline 7 & 15 & 4 & .300 & .378 & -37 \\
\hline 7 & 15 & -4 & 1.30 & -1.527 & .497 \\
\hline 7 & 17 & -4 & 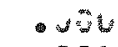 & -.601 & .67 \\
\hline 7 & 18 & -4 & $\therefore c$ & .766 & -.76 \\
\hline 8 & 0 & 4 & 1.760 & -1.896 & .136 \\
\hline 8 & 0 & -4 & 4.678 & 4.427 & \\
\hline 8 & 2 & 4 & 836 & .811 & .0 \\
\hline 8 & 2 & -4 & 2.048 & -1.631 & -.4 \\
\hline 8 & 4 & 4 & 3.266 & 3.238 & .028 \\
\hline 8 & 4 & -4 & 1.794 & $-1.59 z$ & -.27 \\
\hline 8 & 6 & 4 & 2.357 & 2.480 & -.123 \\
\hline 8 & 6 & -4 & 5.355 & -5.229 & -.12 \\
\hline 8 & 8 & 4 & .000 & .458 & -.458 \\
\hline 8 & 8 & -4 & .989 & .768 & .22 \\
\hline 8 & 10 & 4 & .000 & -415 & \\
\hline 8 & 10 & -4 & 2.989 & 2.776 & .21 \\
\hline 8 & 12 & 4 & .600 & .078 & -.37 \\
\hline 8 & 12 & -4 & .758 & .752 & .32 \\
\hline 8 & 14 & -4 & 3.054 & -5.184 & .13 \\
\hline 8 & 16 & -4 & 1.338 & -1.397 & 1015 \\
\hline 8 & 18 & -4 & 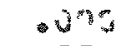 & .86 & -89 \\
\hline 9 & 1 & 4 & .50 & .163 & -16 \\
\hline
\end{tabular}




\begin{tabular}{|c|c|c|c|c|c|}
\hline 9 & 1 & -4 & 4.480 & -4.573 & .093 \\
\hline 9 & 3 & 4 & 1.149 & -1.111 & -.28 \\
\hline 9 & 3 & -4 & 1.186 & .979 & .138 \\
\hline 9 & 5 & 4 & .957 & -1.223 & .366 \\
\hline 9 & 5 & -4 & 2.632 & 2.43 & .189 \\
\hline 9 & 7 & 4 & $.35 t$ & -.285 & .285 \\
\hline 9 & 7 & -4 & .963 & -1.160 & 198 \\
\hline 9 & 9 & 4 & .00 & $-48 i$ & .481 \\
\hline 9 & 9 & -4 & 1.521 & -1.478 & -243 \\
\hline 9 & 11 & 4 & 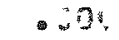 & -.124 & .124 \\
\hline 9 & 11 & -4 & 2.834 & -2.711 & $-12 z$ \\
\hline 9 & 13 & -4 & .000 &.$c 89$ & -.89 \\
\hline 9 & 15 & -4 & 1.450 & 1.333 & .112 \\
\hline 9 & 17 & -4 & ano & .546 & -.546 \\
\hline 9 & 19 & -4 & 1.279 & -1.398 & $-12^{n}$ \\
\hline 10 & 0 & 4 & 1.486 & 1.641 & -.556 \\
\hline 10 & 0 & -4 & 4.868 & -4.975 & .136 \\
\hline 10 & 2 & 4 & .919 & .809 & - 1111 \\
\hline$\because 10$ & 2 & -4 & .000 & -5.156 & 5.156 \\
\hline 10 & 4 & 4 & .850 & -.961 & .211 \\
\hline$* 10$ & 4 & -4 & $.30 \mathrm{C}$ & 1.309 & -1.309 \\
\hline 10 & 6 & 4 & .649 & -.921 & .272 \\
\hline *10 & 6 & -4 & .000 & 2.687 & -2.687 \\
\hline 10 & 8 & 4 & .000 & .445 & -.445 \\
\hline 210 & et & -4 & .000 & -2.955 & 2.955 \\
\hline$=10$ & 10 & -4 & $\cdot \cos$ & -6.602 & 6.602 \\
\hline$* 10$ & 12 & -4 & .000 & -1.861 & 1.861 \\
\hline$* 10$ & 14 & -4 & .20 & 207 & -2.47 \\
\hline *10 & 16 & -4 & $.0 n u$ & -.326 & .326 \\
\hline$* 10$ & 18 & -4 & .200 & -1.313 & 1.312 \\
\hline 11 & 1 & 4 & .290 & .515 & -.515 \\
\hline 11 & 1 & -4 & 3.543 & 3.315 & .32 \\
\hline 11 & 3 & 4 & .500 & .522 & -.52 \\
\hline 11 & 3 & -4 & .307 & -.499 & .193 \\
\hline 11 & 5 & 4 & $.00 r$ & .352 & -35 \\
\hline 11 & 5 & -4 & 2.110 & -1.890 & -.21 \\
\hline 11 & 7 & -4 & .596 & -.382 & -.21 \\
\hline 11 & 9 & -4 & 3.918 & 3.893 & .327 \\
\hline 1 & 11 & -4 & 1.808 & 2.368 & $-.56 ?$ \\
\hline 11 & 13 & -4 & .000 & $-.38 s$ & .38 \\
\hline 11 & 15 & -4 & 1.354 & -1.756 & .132 \\
\hline$\pi$ & 17 & -4 & 1.328 & $i .315$ & $-\infty 1$ \\
\hline 12 & 0 & -4 & $6.45 t$ & 7.227 & -.82 \\
\hline 12 & 2 & -4 & 2.365 & .826 & .24 \\
\hline 12 & 4 & -4 & 2.619 & $-2 \cdot 399$ & -.22 \\
\hline 12 & 6 & -4 & 4.172 & -3.802 & -.37 \\
\hline 12 & 8 & -4 & $4.14 i$ & 4.029 & .11 \\
\hline 12 & 10 & -4 & 4.567 & 4.716 & -.14 \\
\hline 12 & 12 & -4 & -200 & .579 & -.57 \\
\hline 12 & 14 & -4 & $2 \cdot \div 18$ & -1.895 & -.123 \\
\hline 12 & 16 & -4 & .000 & -.427 & 42 \\
\hline 13 & 1 & -4 & 1.578 & -1.408 & -.09 \\
\hline 13 & 3 & -4 & 1.537 & -1.563 & .12 \\
\hline 13 & 5 & -4 & .896 & .413 & .28 \\
\hline 13 & 7 & -4 & .000 & -.061 & .60 \\
\hline 13 & 9 & -4 & 1.795 & -1.798 & -.687 \\
\hline 13 & 11 & -4 & 1.649 & -1.835 & .18 \\
\hline 13 & 13 & -4 & .006 & .451 & -.45 \\
\hline 13 & 15 & -4 & - v6l & .069 & $=0.60$ \\
\hline 14 & 0 & -4 & .278 & $-1.21=$ & .83 \\
\hline$* 14$ & 2 & -4 & -230 & .218 & -.21 \\
\hline$* 14$ & is & -4 & .000 & 5.469 & -3.46 \\
\hline
\end{tabular}




\begin{tabular}{|c|c|c|c|c|c|}
\hline$\div 14$ & 6 & -4 & .000 & 2.215 & -2.215 \\
\hline *14 4 & 8 & -4 & - cor & -.256 & .256 \\
\hline$* 14$ & 10 & -4 & .600 & -.961 & .981 \\
\hline 14 & 12 & -4 & $.00 \mathrm{C}$ & .279 & -279 \\
\hline *14 & 14 & -4 & .00 & 1.788 & -1.788 \\
\hline 15 & 1 & -4 & .000 & .632 & -.632 \\
\hline 15 & 3 & -4 & .500 & .011 & -.411 \\
\hline 15 & 5 & -4 & 1.438 & -1.168 & $.13 n$ \\
\hline 15 & 7 & -4 & .949 & $-1.07^{\prime}$ & .123 \\
\hline 15 & 8 & -4 & 1.122 & 1.306 & $-.18 z$ \\
\hline 15 & 11 & -4 & .714 & .974 & -269 \\
\hline 15 & 13 & -4 & .000 & .038 & -.038 \\
\hline 16 & 0 & -4 & 3.38 & -.036 & .650 \\
\hline 16 & 2 & -4 & 1.313 & 1.347 & -.034 \\
\hline 16 & 4 & -4 & .737 & .590 & .147 \\
\hline 16 & 6 & -4 & .200 & $.28=$ & $-28:$ \\
\hline 16 & 8 & -4 & 1.521 & 1.532 & -.031 \\
\hline 16 & 10 & -4 & 1.714 & 1.784 & -.070 \\
\hline 16 & 12 & -4 & 1.269 & 1.209 & .62 \\
\hline 17 & 1 & -4 & 1.973 & -2.206 & .233 \\
\hline 17 & 3 & -4 & 1.177 & -1.243 & .366 \\
\hline 17 & 5 & -4 & .000 & -.152 & -15 \\
\hline 17 & 7 & -4 & .831 & -.914 & $.33 ?$ \\
\hline 17 & 9 & -4 & 1.165 & -1.845 & .032 \\
\hline 18 & 0 & -4 & $.62 \varepsilon$ & -.519 & -109 \\
\hline$\div 18$ & 2 & -4 & .200 & -.289 & .289 \\
\hline$\div 18$ & 4 & -4 & .000 & .425 & -.425 \\
\hline$* 18$ & 6 & -4 & $\cos 0$ & .206 & -.256 \\
\hline 0 & 0 & 5 & 1.531 & $1.68 \%$ & -.149 \\
\hline 0 & 2 & 5 & 6.166 & 6.383 & -.217 \\
\hline 0 & 4 & 5 & .580 & .597 & -.217 \\
\hline 0 & 6 & 5 & .782 & -.821 & .038 \\
\hline 0 & 8 & 5 & 1.698 & 1.639 & .25 \\
\hline 0 & 10 & 5 & $6.2: 16$ & 0.561 & -.354 \\
\hline 0 & 12 & 5 & 2.011 & 2.598 & -.586 \\
\hline 0 & 14 & 5 & .000 & -.398 & .398 \\
\hline 0 & 16 & 5 & - uno & .197 & -.19 \\
\hline 0 & 18 & 5 & 1.651 & 1.586 & .04 \\
\hline 1 & 1 & 5 & .465 & .365 & .09 \\
\hline 1 & 1 & -5 & .302 & -.171 & -.131 \\
\hline 1 & 3 & 5 & .712 & .706 & .236 \\
\hline 1 & 3 & -5 & .292 & .128 & .163 \\
\hline 1 & 5 & 5 & .000 & .08 & $-.03 \%$ \\
\hline 1 & 5 & -5 & 1.090 & -1.162 & .073 \\
\hline 1 & 7 & 5 & 1.728 & 1.671 & .257 \\
\hline 1 & 7 & -5 & 1.765 & 1.957 & -.192 \\
\hline 1 & 9 & 5 & 2.068 & -1.895 & -.173 \\
\hline 1 & 9 & -5 & 1.880 & -1.847 & -.033 \\
\hline 1 & 11 & 5 & .320 & .545 & -.545 \\
\hline 1 & 11 & -5 & .300 & .423 & -423 \\
\hline 1 & 13 & 5 & .845 & .707 & .13 \\
\hline 1 & 13 & -5 & .550 & -.693 & .16 \\
\hline 1 & 15 & 5 & .200 & .737 & -.737 \\
\hline 1 & 15 & -5 & .200 & $.18 \%$ & -.180 \\
\hline 1 & 17 & 5 & 1.213 & -1.184 & -.02 \\
\hline 1 & 17 & -5 & .970 & -.346 & .346 \\
\hline 1 & 19 & -5 & D. 00 & $.34 J$ & -.34 \\
\hline 2 & 0 & 5 & 3.588 & $-z .525$ & -.033 \\
\hline 2 & 0 & -5 & 5.972 & -6.590 & .688 \\
\hline 2 & 2 & 5 & -.30 & -.172 & .172 \\
\hline 2 & 2 & -5 & 1.749 & -1.651 & -.39 \\
\hline 2 & 4 & 5 & $.6 n z$ & .438 & .16 \\
\hline
\end{tabular}




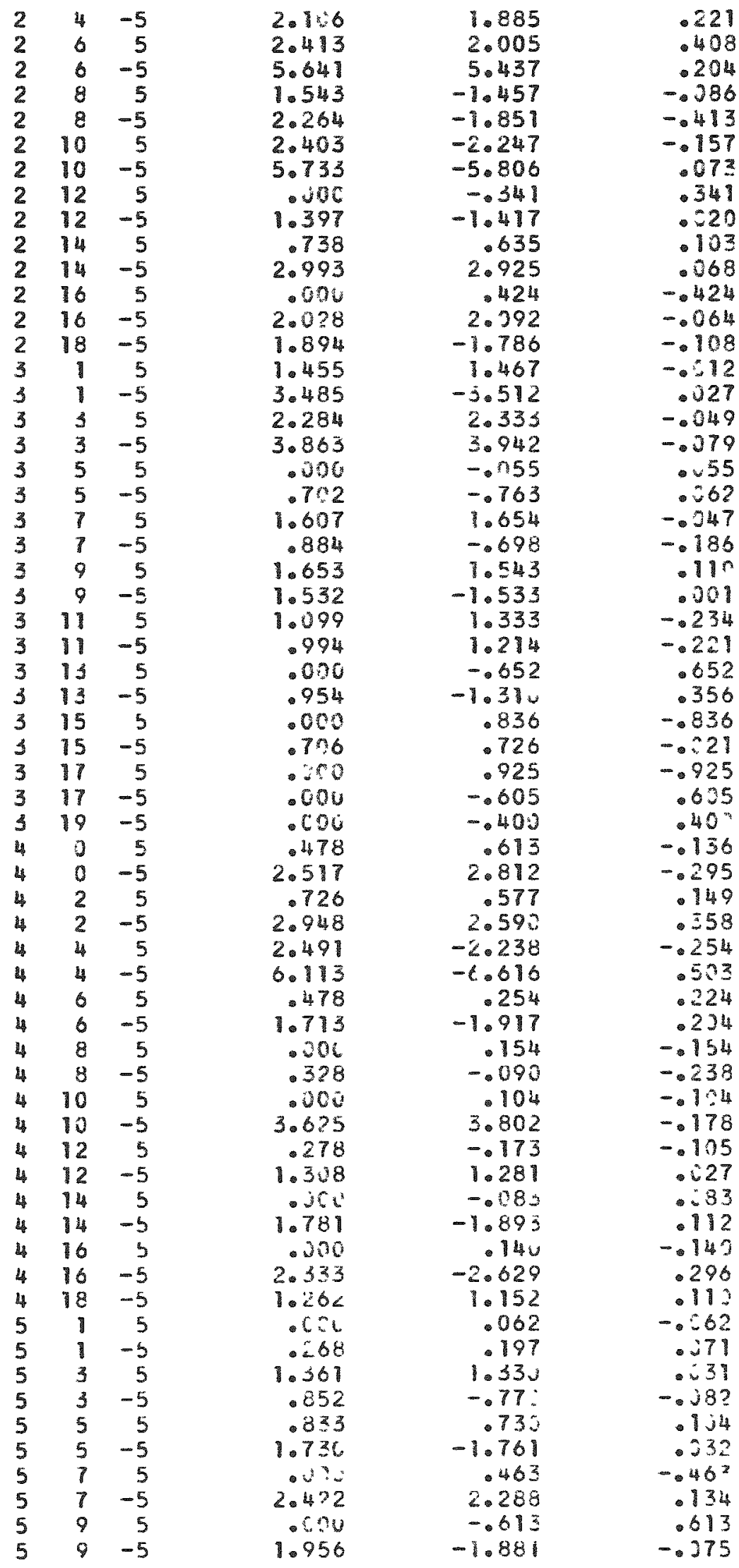




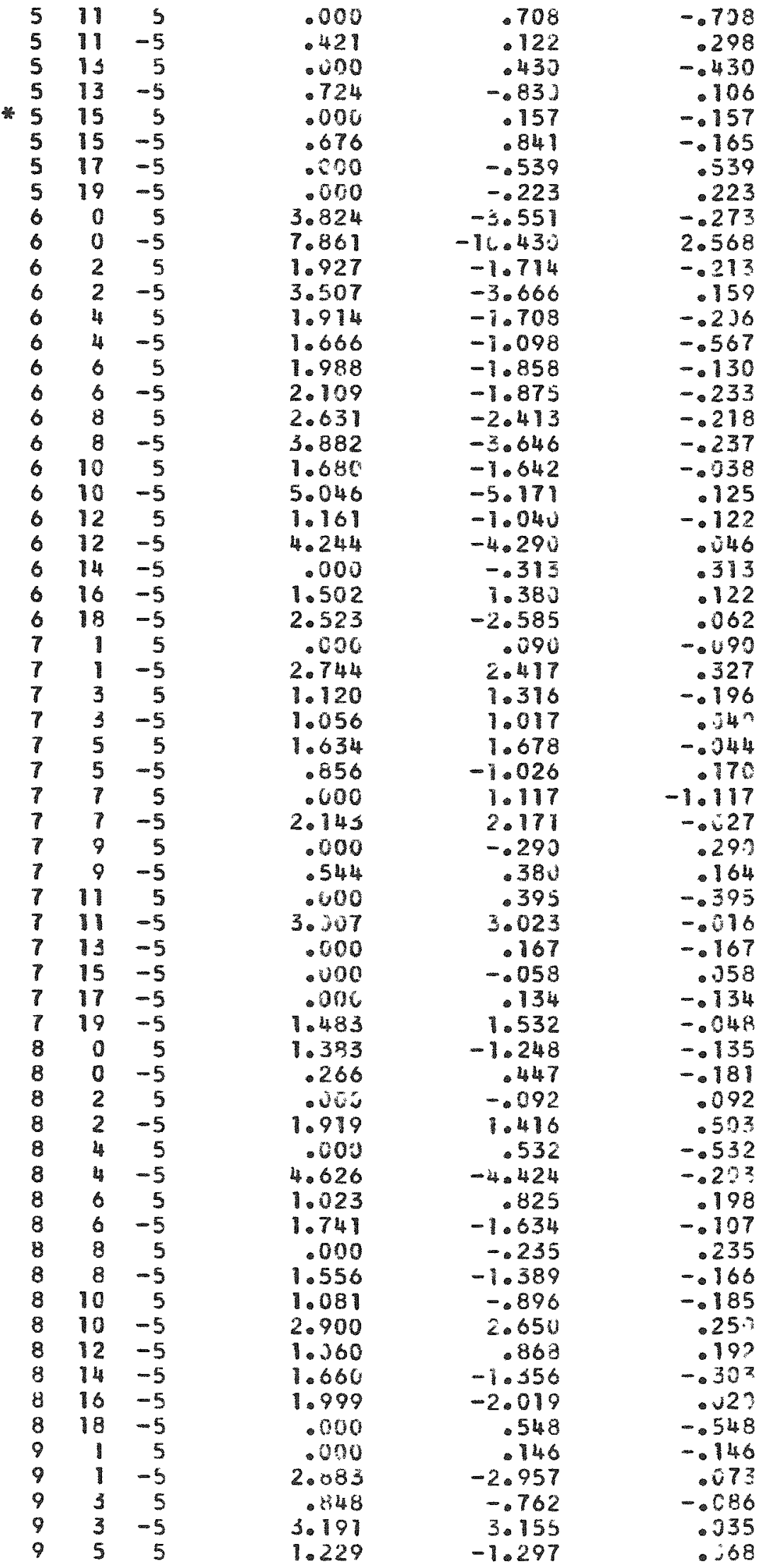




\begin{tabular}{|c|c|c|c|c|c|}
\hline 9 & 5 & -5 & .000 & 2.156 & $-2 \cdot 156$ \\
\hline 9 & 7 & 5 & .000 & -.434 & .434 \\
\hline 9 & 7 & -5 & .009 & .425 & -.425 \\
\hline 9 & 9 & -5 & 2.280 & -2.436 & .155 \\
\hline 9 & 11 & -5 & .000 & -.221 & .221 \\
\hline 9 & 13 & -5 & 1.574 & 1.092 & -.019 \\
\hline 9 & 15 & -5 & 1.941 & 2.05 & -.364 \\
\hline 9 & 17 & -5 & .601 & $-87 \%$ & .269 \\
\hline 10 & 0 & 5 & 1.237 & 1.061 & .177 \\
\hline 10 & 0 & -5 & 5.354 & -5.136 & -.218 \\
\hline 10 & 2 & 5 & .000 & .676 & -.676 \\
\hline 10 & 2 & -5 & 1.562 & -1.336 & -.227 \\
\hline 10 & 4 & -5 & 2.576 & 2.427 & .149 \\
\hline 10 & 6 & -5 & 2.461 & 2.107 & .353 \\
\hline 10 & 8 & -5 & 2.659 & -2.432 & -.227 \\
\hline 10 & 10 & -5 & 3.134 & -3.067 & $-.06 ?$ \\
\hline 10 & 12 & -5 & .000 & .324 & -324 \\
\hline 10 & 14 & -5 & .600 & -844 & -.844 \\
\hline 10 & 16 & -5 & .000 & .835 & -.835 \\
\hline 10 & 18 & -5 & .200 & -.983 & .981 \\
\hline 11 & 1 & -5 & 2.404 & 2.254 & $.15^{\prime \prime}$ \\
\hline 11 & 3 & -5 & .438 & -.293 & -.145 \\
\hline 11 & 5 & -5 & .898 & -.855 & -.344 \\
\hline 1 & 7 & -5 & 2.202 & 2.327 & -.125 \\
\hline i1 & 9 & -5 & 1.320 & 1.664 & .162 \\
\hline 11 & 11 & -5 & .664 & .845 & -.181 \\
\hline 11 & 13 & -5 & $.8 \pi 9$ & -.863 & .054 \\
\hline 11 & 15 & -5 & .000 & .655 & -.655 \\
\hline 1 & 17 & -5 & .000 & .929 & -.929 \\
\hline 12 & 0 & -5 & 2.650 & 2.647 & .004 \\
\hline 12 & 2 & -5 & 3.192 & 3.034 & .158 \\
\hline 12 & 4 & -5 & .055 & $-.26=$ & -.395 \\
\hline 12 & 6 & -5 & 2.579 & 1.997 & .482 \\
\hline 12 & 8 & -5 & 2.635 & 20689 & -.054 \\
\hline 12 & 10 & -5 & 2.193 & 2.213 & -.017 \\
\hline 12 & 12 & -5 & .000 & .551 & -.551 \\
\hline 12 & 14 & -5 & 1.028 & 1.039 & -010 \\
\hline 12 & 16 & -5 & .893 & .863 & .629 \\
\hline 13 & 1 & -5 & 2.354 & $-2 \cdot 353$ & - - उी" \\
\hline 13 & 3 & -5 & - UDC & -166 & .166 \\
\hline 13 & 5 & -5 & .000 & -429 & .429 \\
\hline 13 & 7 & -5 & 1.968 & -1.756 & -.212 \\
\hline 13 & 9 & -5 & 2.191 & $-2 \cdot 23=$ & .642 \\
\hline 13 & 11 & -5 & .300 & -.224 & .224 \\
\hline 13 & 13 & -5 & .200 & -.483 & $.48^{\prime \prime}$ \\
\hline 13 & 15 & -5 & .235 & -.707 & .707 \\
\hline 14 & 0 & -5 & 1.033 & 8.111 & -.108 \\
\hline 14 & 2 & -5 & .05 & .755 & -.755 \\
\hline 14 & 4 & -5 & .304 & -.176 & .176 \\
\hline 14 & 6 & -5 & $a n$ & -.107 & .113 \\
\hline 14 & 8 & -5 & - טvo & $.35^{\circ}$ & -.350 \\
\hline 14 & 10 & -5 & .000 & .37. & -.373 \\
\hline 14 & 12 & -5 & .000 & .504 & -.524 \\
\hline 14 & 14 & -3 & .000 & 0.544 & -.644 \\
\hline 15 & 1 & -5 & .525 & .669 & -.144 \\
\hline 15 & 3 & -5 & .000 & -.323 & .323 \\
\hline 15 & 5 & -5 & .672 & -.717 & .045 \\
\hline 15 & 7 & -5 & .00 & .469 & -.469 \\
\hline 15 & 9 & -5 & .800 & .321 &.- .21 \\
\hline 15 & 11 & -5 & .000 & .112 & -.112 \\
\hline 15 & 13 & -5 & $-3 n n^{2}$ & -.235 & .235 \\
\hline 16 & 0 & -5 & 1.341 & -1.389 & .048 \\
\hline
\end{tabular}




\begin{tabular}{|c|c|c|c|c|c|}
\hline & & & & & \\
\hline 16 & 2 & -5 & .000 & .323 & -.323 \\
\hline 16 & 4 & -5 & .000 & 1.248 & -1.248 \\
\hline 16 & 6 & -5 & .000 & .652 & -652 \\
\hline 16 & 8 & -5 & .600 & -.567 & .567 \\
\hline 16 & 10 & -5 & .000 & -122 & .122 \\
\hline 16 & 12 & -5 & - $40^{\circ}$ & -.001 & .001 \\
\hline 17 & 1 & -5 & .950 & -.963 & .033 \\
\hline 17 & 3 & -5 & .000 & -.310 & .212 \\
\hline 17 & 3 & -5 & - vou & -295 & .295 \\
\hline 17 & 7 & -5 & .641 & -.856 & 215 \\
\hline 17 & 8 & -5 & 1.173 & -1.263 & .087 \\
\hline 18 & 0 & -5 & .00 & -253 & 253 \\
\hline 18 & 2 & -5 & .600 & -.529 & .529 \\
\hline 18 & 4 & -5 & $-4 n_{c}$ & -.799 & .799 \\
\hline 18 & 6 & -3 & -00 & -.748 & .748 \\
\hline 18 & 8 & -5 & .000 & -.697 & .697 \\
\hline *19 & 1 & -5 & .300 & .373 & $-.37 n$ \\
\hline 19 & 3 & -5 & 1.197 & 1.377 & -18 \\
\hline 0 & 0 & 6 & 6.152 & 6.895 & -.74 \\
\hline 0 & 2 & 6 & 3.276 & 3.321 & -.345 \\
\hline 0 & 4 & 6 & 2.425 & -2.117 & -.288 \\
\hline 0 & 6 & 6 & 3.112 & -2.867 & -.24 \\
\hline 0 & 8 & 6 & 3.136 & 4.659 & -1.523 \\
\hline 0 & 10 & 6 & 4.475 & 4.863 & -39 \\
\hline 0 & 12 & 6 & 1.043 & 1.173 & -.12 \\
\hline 0 & 14 & 6 & 1.526 & -1.537 & .01 \\
\hline 0 & 16 & 6 & .006 & .044 & -.04 \\
\hline 1 & 1 & 6 & .621 & .672 & -.5 \\
\hline 1 & 1 & -6 & 2.217 & $-2.10 \mathrm{~J}$ & -.11 \\
\hline 1 & 3 & 6 & 3.127 & -2.965 & -16 \\
\hline 1 & 3 & -6 & .050 & -.188 & .18 \\
\hline 1 & 5 & 6 & 2.355 & -2.743 & -.11 \\
\hline 1 & 5 & -6 & .000 & $-.29 v$ & .29 \\
\hline 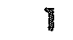 & 7 & 6 & .020 & -.071 & .07 \\
\hline 1 & 7 & -6 & 1.231 & -1.075 & -.15 \\
\hline 1 & 9 & 6 & .600 & -202 & .20 \\
\hline 1 & 9 & -6 & 1.698 & -1.501 & -.19 \\
\hline 1 & 11 & 6 & .751 & -.762 & .06 \\
\hline 1 & 11 & -6 & .30 & -.475 & .47 \\
\hline 1 & 13 & 6 & .035 & -.704 & .06 \\
\hline 1 & 13 & -6 & .000 & -164 & .16 \\
\hline 1 & 15 & 6 & 1.487 & -1.302 & -.18 \\
\hline 1 & 15 & -3 & .000 & -303 & .30 \\
\hline 1 & 17 & -6 & .000 & .081 & -.38 \\
\hline 2 & 0 & 6 & 2.106 & $-\approx-142$ & .23 \\
\hline 2 & 0 & -6 & 6.802 & -8.064 & 1.26 \\
\hline 2 & 2 & 6 & 630 & .023 & -.32 \\
\hline 2 & 2 & -6 & 3.636 & $-248 f_{i}$ & -.18 \\
\hline 2 & 4 & 6 & 4.242 & 4.134 & .10 \\
\hline 2 & 4 & -6 & 2.693 & 2.297 & \\
\hline 2 & 6 & 6 & 2.810 & $\therefore 501$ & .2 \\
\hline 2 & 6 & -6 & 1.896 & 1.605 & 2 \\
\hline 2 & 8 & 6 & .40 & -.319 & \\
\hline 2 & 8 & -5 & 4.787 & $-4.56 j$ & -.2 \\
\hline 2 & 10 & 6 & 1.434 & $-1.4,57$ & 0 \\
\hline 2 & 10 & -6 & 5.583 & -5.786 & \\
\hline 2 & 12 & 6 & - vact & - & -.5 \\
\hline 2 & 12 & -6 & .020 & -.509 & \\
\hline 2 & 14 & 6 & 1.380 & 1.328 & . \\
\hline 2 & 14 & -6 & 1.442 & 1.302 & \\
\hline 2 & 16 & -6 & $\therefore O C$ & -.461 & \\
\hline 2 & 18 & -6 & 2.242 & -1.973 & \\
\hline
\end{tabular}




\begin{tabular}{|c|c|c|c|c|c|}
\hline 3 & 1 & 6 & .597 & -.638 & .341 \\
\hline *3 & 1 & -6 & $-\infty$ & -193 & $.19 \%$ \\
\hline 3 & 3 & 6 & $\therefore \mathrm{Cu}$ & .591 & -.591 \\
\hline$\# 3$ & 3 & -6 & - ordi & .218 & -.218 \\
\hline 3 & 5 & 6 & .589 & -.265 & -.125 \\
\hline$* 3$ & 5 & -6 & $0: 3$ & -1.749 & 1.749 \\
\hline 3 & 7 & 6 & .432 & -.477 &.- .15 \\
\hline$* 3$ & 7 & -6 & $.00 \overrightarrow{0}$ & .173 & -.178 \\
\hline 3 & G & 6 & .0 & .417 & -.417 \\
\hline$* 3$ & 9 & -6 & .65 & .742 & -.742 \\
\hline 3 & 11 & 6 & -382 & -.296 & -.80 \\
\hline *3 & 11 & -6 & $\therefore 00$ & -.184 & .184 \\
\hline 3 & 13 & 6 & 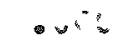 & .26 & -.867 \\
\hline$* 3$ & 13 & -6 & .360 & -.281 & .231 \\
\hline 3 & 15 & 6 & .00 & .124 & -.124 \\
\hline$* 3$ & 15 & -6 & -80 & -.208 & $-2=9$ \\
\hline$* 3$ & 11 & -6 & $0=8$ & -.021 & .221 \\
\hline 4 & 0 & 6 & 4.959 & $\cong 543$ & $-.58 ?$ \\
\hline 4 & 0 & -6 & 3.273 & 2.974 & .299 \\
\hline 4 & 2 & 6 & 2.107 & $\therefore 46$ & .121 \\
\hline 4 & 2 & -6 & .541 & -.119 & -422 \\
\hline 4 & 4 & 6 &.-200 & -.175 & .175 \\
\hline 4 & 4 & -6 & 4.583 & -4.579 & $\cdots \cdots$ \\
\hline 4 & 6 & 6 & 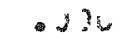 & .673 & -.673 \\
\hline 4 & 6 & -6 & 4.234 & -.786 &. - \pm 34 \\
\hline 4 & 8 & 6 & 2.854 & 2.735 & $12^{\circ}$ \\
\hline 4 & 8 & -6 & 2.269 & $\therefore .009$ & $-56^{\circ}$ \\
\hline 4 & 10 & 6 & 2.421 & $\therefore 271$ & $.75^{\circ}$ \\
\hline 4 & 10 & -6 & 2.266 & 1.975 & $.59^{2}$ \\
\hline 费 & 12 & 6 & 1.995 & 1.951 & $.3^{24}$ \\
\hline 4 & 12 & -6 & .45 & -.429 & -336 \\
\hline 4 & 14 & -6 & 3.446 & $-\therefore=8$. & -9.67 \\
\hline 4 & 16 & -6 & .080 & -.297 & .097 \\
\hline 4 & 18 & -6 & 1.336 & 1.184 & .15 \\
\hline 5 & 1 & 6 & .6 .35 & -.577 &.- .537 \\
\hline 5 & 1 & -6 & $-4=1$ & -.397 & -.235 \\
\hline 5 & 3 & 6 & .500 & -.063 & $.50^{2}$ \\
\hline 5 & 2 & -6 & $.8 \cdot 3<$ & .067 & .135 \\
\hline$* 3$ & 5 & 6 & $-6=3$ & 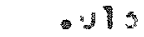 & $-05: ?$ \\
\hline$* 5$ & 5 & -6 & -50 & .36 & $-.36 ?$ \\
\hline 5 & 9 & 6 & 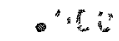 & -.50 & .959 \\
\hline 5 & 9 & -6 & .498 & $-27=$ & -.215 \\
\hline 3 & 7 & 6 & .428 & -.534 & -.294 \\
\hline 5 & 7 & -6 & 1.351 & $.76=$ & .291 \\
\hline 5 & 11 & 6 & - $40:$ & $-.20 d$ & $.2: 3$ \\
\hline 5 & 11 & -6 & -4 & .133 & -133 \\
\hline 5 & 13 & -6 & -20 & .168 & -.169 \\
\hline 5 & 15 & -6 & .559 & $.44 \mathrm{~J}$ & .19 \\
\hline 5 & 17 & -6 & $-3 y$ & .480 & -.486 \\
\hline 6 & 0 & 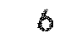 & 1.487 & -1.506 & 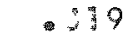 \\
\hline 6 & B & -6 & 4.744 & $-4 \cdot 480$ & -34? \\
\hline 6 & 2 & 6 & $1 . .53$ & -.928 & -.125 \\
\hline 6 & 2 & -6 & $\alpha x^{\circ}$ & -1.629 & -.71 \\
\hline 6 & 4 & 6 & $\therefore 20$ & -358 & -.389 \\
\hline 6 & 4 & -6 & 2.65 & -242 & $-.233^{\prime}$ \\
\hline 6 & 6 & 6 & 13 & -.269 & $\therefore \approx 69$ \\
\hline 6 & $b$ & -6 & $\therefore u$ & .012 & -.312 \\
\hline 6 & 8 & 6 & $.0 r 0$ & -.504 & - 3.3 \\
\hline 6 & 8 & -6 & 1.897 & -3.759 & -.139 \\
\hline 6 & 12 & 6 & 1.599 & $-i: 179$ &.$-: 2$ \\
\hline 6 & 11) & -6 & 4.024 & $-.77 t$ & -847 \\
\hline 6 & 12 & -6 & 2.142 & -1.861 & $-28 ?$ \\
\hline
\end{tabular}




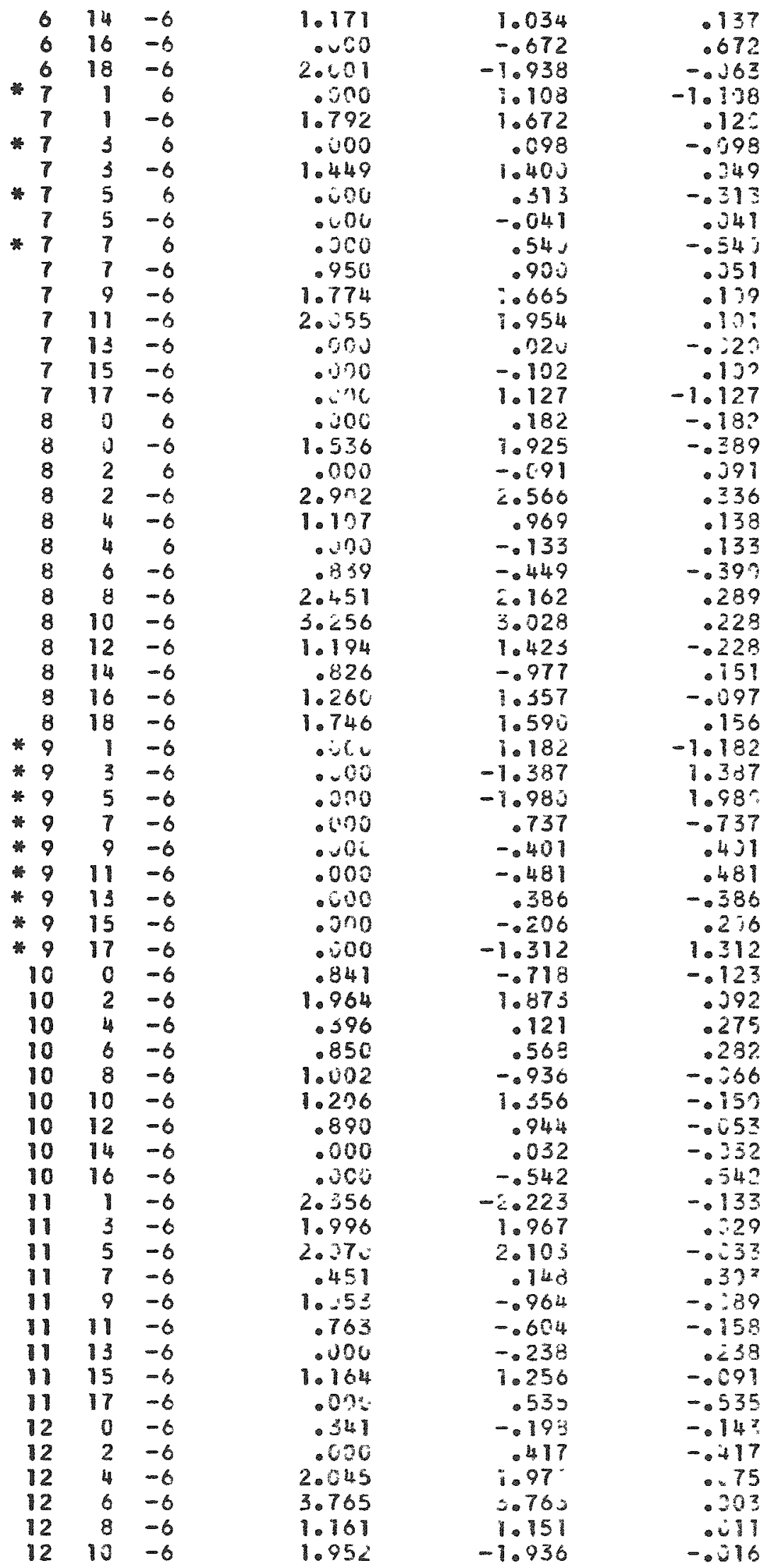




\begin{tabular}{|c|c|c|c|c|c|}
\hline 12 & 12 & -6 & $.00 \%$ & .527 & -.527 \\
\hline 12 & 14 & -6 & 2.965 & 2.078 & -.025 \\
\hline 12 & 16 & -6 & 1.190 & 1.296 & -.106 \\
\hline 13 & 1 & -6 & .841 & .839 & .092 \\
\hline 13 & 3 & -6 & 1.716 & -1.685 & -.037 \\
\hline 13 & 5 & -6 & $3.4 \div$ & $-5 \cdot 288$ & -125 \\
\hline 13 & 7 & -6 & 1.316 & -1.283 & -.036 \\
\hline 13 & 9 & -6 & - $v$ & .538 & -.530 \\
\hline 13 & 11 & -6 & .557 & .560 & -.311 \\
\hline 13 & 13 & -6 & 2.111 & -2.045 & $-\infty 65$ \\
\hline 13 & 15 & -6 & 1.943 & -1.81 & -.33 \\
\hline 14 & 0 & -6 & 1.526 & 1.379 & -.253 \\
\hline 14 & 2 & -6 & .637 & .619 & .48 \\
\hline 14 & 4 & -6 & 3.928 & -4.018 & .090 \\
\hline 14 & 6 & -6 & $3 .-44$ & -3.994 & .449 \\
\hline 14 & 8 & -6 & .606 & .233 & -.233 \\
\hline 14 & 10 & -6 & 2.127 & 2.044 & .463 \\
\hline 14 & 12 & -6 & $1.4 \leq 5$ & -1.352 &.- .85 \\
\hline 14 & 14 & -6 & 2.187 & -2.147 & -347 \\
\hline$\approx 15$ & 1 & -6 & .600 & .497 & -497 \\
\hline *15 & 3 & -6 & $.0 t u$ & .973 & -973 \\
\hline *15 & 5 & -6 & .320 & 2.108 & -2.128 \\
\hline *15 & 7 & -6 & ה, & $2.04 w$ & $-2 .: 46$ \\
\hline * 15 & 9 & -6 & .000 & -.474 & .474 \\
\hline * 15 & 11 & -6 & .60 & -.351 & .351 \\
\hline$* 5$ & 13 & -6 & . Dit: & .951 & -.951 \\
\hline 16 & 3 & -6 & 2.359 & -2.573 & .214 \\
\hline 16 & 2 & -6 & .836 & -.802 & -.534 \\
\hline 16 & 4 & -6 & $1.27 \%$ & 7.155 & .117 \\
\hline 16 & 6 & -6 & 1.193 & .710 & .382 \\
\hline 16 & 8 & -6 & 1.367 & -1.373 & .008 \\
\hline 16 & 13 & -6 & 1.457 & -1.537 & .079 \\
\hline 16 & 12 & -6 & - wel & -.319 & .319 \\
\hline 17 & 1 & -0 & 1.360 & 1.517 & -.157 \\
\hline 17 & 3 & -6 & .000 & .482 & $-.48^{\circ}$ \\
\hline 17 & 5 & -6 & 1.282 & -1.339 & .458 \\
\hline 17 & 7 & -6 & - obic & -.864 & .664 \\
\hline 17 & 9 & -6 & 1.255 & 1.235 &. .22 \\
\hline 18 & 0 & -6 & .803 & .788 & .015 \\
\hline 18 & 2 & -6 & .96 & .367 &.- .67 \\
\hline 18 & 4 & -6 & 1.466 & -1.732 & .264 \\
\hline 18 & 6 & -6 & $.010:$ & -1.054 & 1.054 \\
\hline 18 & 8 & -6 & .046 & -.19 & .194 \\
\hline$\div 19$ & 1 & -6 & $0.0^{3} y$ & .17. & -.172 \\
\hline *19 & 3 & -6 & .006 & 1.777 & -1.777 \\
\hline 0 & 0 & 7 & .4724 & .288 & .116 \\
\hline 0 & 2 & 7 & .590 & -500 & .509 \\
\hline 0 & 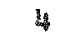 & 7 & $.4 C:$ & -.142 & .042 \\
\hline 0 & 6 & 7 & $-3 t^{2}$ & -639 & .639 \\
\hline$* 0$ & 8 & 7 & 0 & -.062 & $.06 ?$ \\
\hline 0 & 10 & 7 & .00 & .251 & -.251 \\
\hline 0 & 12 & 7 & $\cdot 0_{0}$ & .171 & -.171 \\
\hline 0 & 14 & 7 & .00 & .63 & -932 \\
\hline 1 & 1 & 7 & 1. $462^{\circ}$ & -1.412 & $-.55=$ \\
\hline 1 & 1 & -7 & $.64 !$ & .648 & -.328 \\
\hline 1 & 3 & 7 & .892 & $-1 \cdot(1)$ & .123 \\
\hline 1 & 3 & -7 & .982 & -1.143 & .16 ? \\
\hline 1 & 5 & 8 & -90 & -.127 & 0.27 \\
\hline 1 & 5 & -7 & 0 & -.512 & $.41^{\circ}$ \\
\hline I & 7 & 7 & .865 & -7.056 & - 191 \\
\hline 1 & 7 & -7 & .354 & -.632 & .078 \\
\hline 1 & 9 & 7 & .415 & -.479 & .05 \\
\hline
\end{tabular}




\begin{tabular}{|c|c|c|c|c|c|}
\hline 1 & 9 & -7 & .300 & .274 & -.274 \\
\hline $\mathbb{1}$ & 11 & 7 & 1.276 & -7.102 & .226 \\
\hline 1 & 11 & -7 & .00 & -179 & .176 \\
\hline 1 & 13 & 7 & .030 & -.493 & .493 \\
\hline 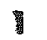 & 13 & -7 & .856 & .918 & -339 \\
\hline 1 & 15 & -7 & .100 & -.238 & .238 \\
\hline 2 & $J$ & 7 & 1.110 & -.986 & -.123 \\
\hline 2 & 3 & -7 & 1.801 & -1.818 & .317 \\
\hline 2 & 2 & 7 & 1.496 & -1.409 & $-\therefore 87$ \\
\hline 2 & 2 & -7 & 2.823 & -4.567 & -.256 \\
\hline 2 & 4 & 7 & .432 & .444 & -.342 \\
\hline 2 & 4 & -7 & $1.27 \%$ & -3.349 & .59 \\
\hline 2 & 6 & 7 & .00 & .655 & -.655 \\
\hline 2 & 6 & -7 & 4.511 & -4.199 & -112 \\
\hline 2 & 8 & 7 & .020 & -.454 & .454 \\
\hline 2 & 8 & -7 & 2.199 & -2.159 & -.041 \\
\hline 2 & 10 & 7 & 1.848 & -1.679 & -.169 \\
\hline 2 & 10 & -7 & .533 & -.521 & -12 \\
\hline 2 & 12 & 7 & .601 & -1.005 & -404 \\
\hline 2 & 12 & -7 & .891 &.- .062 & .171 \\
\hline 2 & 14 & -7 & 2.134 & -2.085 & -349 \\
\hline 2 & 16 & -7 & 1.476 & -1.46 .3 & -.315 \\
\hline 3 & 1 & 7 & .000 & -.116 & .116 \\
\hline 3 & 1 & -7 & .354 & .257 & .346 \\
\hline 3 & 3 & 7 & $-\infty$ & -538 & 639 \\
\hline 3 & 3 & -7 & .000 & -.222 & .222 \\
\hline 3 & 5 & 7 & .276 & -.591 & .316 \\
\hline 3 & 5 & -7 & 1.453 & .385 & .369 \\
\hline 3 & 7 & 7 & -5 & $-.69 x$ & .699 \\
\hline 3 & 7 & -7 & .070 & .486 & -.483 \\
\hline 3 & 9 & 7 & .000 & .254 & -.254 \\
\hline 3 & 9 & -7 & .658 & .746 & -.88 \\
\hline 5 & 11 & 7 & .000 & -.207 & .227 \\
\hline 3 & 11 & -7 & .608 & -.833 & .225 \\
\hline 3 & 13 & -7 & .834 & .928 & -.93 \\
\hline 3 & 15 & -7 & .000 & .257 & -257 \\
\hline 4 & 0 & 7 & 3.481 & 5.140 & .336 \\
\hline 4 & 0 & -7 & .558 & -.613 & .35 \\
\hline 4 & 2 & 7 & 2.47 & 1.917 & .131 \\
\hline 4 & 2 & -7 & 1.453 & -208 & -.245 \\
\hline 4 & 4 & -7 & 2.947 & 2.645 & \pm 02 \\
\hline 4 & 4 & 7 & $1 . .10$ & .807 & $.27 ?$ \\
\hline 4 & 6 & 7 & .40 & .470 & $-.47 \%$ \\
\hline 4 & 6 & -7 & 3.663 & 3.496 & 168 \\
\hline 4 & 8 & 7 & 1.639 & 1.465 & .174 \\
\hline 4 & 8 & -7 & .000 & .253 & -.258 \\
\hline 4 & 12 & 7 & 2.855 & 2.542 & .312 \\
\hline 4 & 10 & -7 & 2.555 & -2.354 & -.201 \\
\hline 4 & 12 & -7 & $.0 n c$ & -.147 & .147 \\
\hline 4 & 14 & -7 & 2.635 & 2.551 & .285 \\
\hline 4 & 16 & -1 & $1 . \leq 2 \leq 4$ & 1.172 & \\
\hline 5 & 1 & 7 & 1.443 & -1.450 & \\
\hline 5 & 1 & -7 & 1.327 & $-i \cdot 4 v=$ & \\
\hline 5 & 3 & 7 & .400 & .327 &.- .227 \\
\hline 5 & 3 & -7 & 1.718 & 3.771 & -.053 \\
\hline 5 & 5 & 7 & .717 & .765 & -.648 \\
\hline 5 & 5 & -7 & 1.465 & 1.455 & $\therefore 1$ \\
\hline 5 & 7 & 7 & $a x^{*} \cdot v$ & -.711 & \\
\hline 5 & 7 & -7 & $2 .<44$ & -2.384 & \\
\hline 5 & 9 & -7 & 0320 & .768 & -.73 \\
\hline 5 & 11 & -7 & - tra & -445 & - \\
\hline 5 & 13 & -7 & .389 & .798 & \\
\hline
\end{tabular}




\begin{tabular}{|c|c|c|c|c|}
\hline 15 & -7 & -200 & -381 & $\begin{array}{r}.387 \\
-\quad 31\end{array}$ \\
\hline 17 & -7 & .400 & .111 & -111 \\
\hline 0 & 7 & 1.621 & -1.467 & -154 \\
\hline 0 & -7 & 5.267 & 5.102 & .165 \\
\hline 2 & 7 & .00 & -342 & .342 \\
\hline 2 & -7 & .567 & .109 & .459 \\
\hline 4 & 7 & 1.822 & 1.586 & .237 \\
\hline 4 & -7 & 1.236 & -1.255 & .41 \\
\hline 6 & -7 & 2.726 & -2.529 & -.89 \\
\hline 8 & -7 & 1.775 & 1.732 & .24 \\
\hline 13 & -7 & 1.629 & 1.639 & $\ldots 1$ \\
\hline 12 & -7 & 1.426 & 1.421 & -5 \\
\hline 14 & -7 & 1.328 & -1.350 & .62 \\
\hline 16 & -7 & 1.788 & -1.767 &.--2 \\
\hline 1 & -7 & .755 & .866 & $-\therefore !$ \\
\hline 3 & -1 & 1.942 & -876 & -.26 \\
\hline 5 & -7 & .712 & .644 & .26 \\
\hline 7 & -7 & 1.279 & 1.049 & .161 \\
\hline 9 & -7 & .535 & .634 & -.12 \\
\hline 11 & -7 & 1.721 & -1.696 & -.32 \\
\hline 13 & -7 & $\div 3:$ & .373 & -.37 \\
\hline 15 & -7 & .000 & .276 & -.276 \\
\hline 17 & -7 & .000 & .967 & $-.96 \%$ \\
\hline 0 & -7 & .959 & -.786 & -.15 \\
\hline 2 & -7 & 1.774 & 1.730 & 23 \\
\hline 4 & -7 & 5.841 & 5.999 & -.15 \\
\hline 6 & -7 & 3.647 & $\therefore .556$ & \\
\hline 8 & -7 & 2.150 & 3.049 & .1 \\
\hline 10 & -7 & .200 & .379 & -.37 \\
\hline 12 & -7 & .620 & -337 & .23 \\
\hline 14 & -7 & 2.661 & 2.903 & -.24 \\
\hline 16 & -1 & 2.713 & -209 & -.49 \\
\hline 1 & -7 & .315 & -.724 & -19 \\
\hline 3 & -7 & 1.874 & -2.269 & .19 \\
\hline 5 & -7 & $1.32 c$ & -1.981 & .16 \\
\hline 7 & -7 & 2.536 & $-2 \cdot 444$ & \\
\hline 9 & -7 & .447 & -.308 & -.13 \\
\hline 11 & -7 & .030 & -.496 & -49 \\
\hline 13 & -1 & 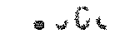 & $.15=$ & $-.15 ?$ \\
\hline 15 & -7 & 1.891 & $-2 \cdot 41$ & .43 \\
\hline 2 & -7 & .443 & -.117 & -.32 \\
\hline 0 & -7 & $4.82 z$ & 4.969 & -14 \\
\hline 4 & -7 & 1.496 & -1.247 &.$-<4$ \\
\hline 6 & -7 & 3.364 & $-\ldots 32$ & $-.2^{2}$ \\
\hline 8 & -7 & .729 & .019 & .1 \\
\hline 10 & -7 & 1.738 & $1.76:$ & -.82 \\
\hline 12 & -7 & 1.821 & 1.871 &.- .2 \\
\hline 14 & -7 & 2.006 & -2.0104 & $-\therefore \mathrm{a}$ \\
\hline 16 & -7 & 2.327 & -2.297 & -53 \\
\hline 1 & -7 & 2.499 & -2.212 & -.28 \\
\hline 3 & -7 & 1.519 & 1.439 & .28 \\
\hline 5 & -7 & 1.912 & 2.142 & -.22 \\
\hline 7 & -7 & .746 & -.876 & 13 \\
\hline 9 & -7 & .722 & -.650 & -.36 \\
\hline 11 & -7 & 1.336 & -1.554 & \\
\hline 13 & -7 & -492 & .684 & -.6 \\
\hline 15 & -7 & 1.562 & 1.161 & -0. \\
\hline $\mathfrak{0}$ & -7 & 2.907 & -2.962 & \\
\hline 2 & -7 & 1.114 & -3.052 & -.26 \\
\hline 4 & -1 & 3.775 & 2.552 & .22 \\
\hline 6 & -7 & $3 . .58$ & 2.998 & 0 \\
\hline $\mathrm{e}$ & -7 & 1.250 & -1.034 & \\
\hline
\end{tabular}




\begin{tabular}{|c|c|c|c|c|c|}
\hline 12 & 10 & -7 & 2.644 & -2.716 & .072 \\
\hline 12 & 12 & -7 & Dod & -336 & .336 \\
\hline 12 & 14 & -7 & 2.251 & 2.127 & 124 \\
\hline 13 & 1 & -7 & .976 & 1.113 & -.138 \\
\hline 13 & 3 & -7 & 1.819 & -1.755 & -.065 \\
\hline 13 & 5 & -7 & 1.354 & -1.303 & -.050 \\
\hline 13 & 7 & -7 & .600 & -323 & .323 \\
\hline 13 & 9 & -7 & .869 & .871 & -.302 \\
\hline 13 & II & -7 & .600 & -.439 & .439 \\
\hline 13 & 13 & -7 & .000 & -.753 & .753 \\
\hline 14 & 0 & -7 & 1.511 & $1 \cdot 483$ & .02 \\
\hline 14 & 2 & -7 & 1.145 & $-1 \cdot 120$ & -.025 \\
\hline 14 & 4 & -7 & 2.016 & -2.026 & $-62^{3}$ \\
\hline 14 & 6 & -7 & 2.929 & $-2 \cdot 886$ & -.043 \\
\hline 14 & 8 & -7 & .776 & $.56 \vec{a}$ & -211 \\
\hline 14 & 10 & -7 & .000 & .166 & -.166 \\
\hline 14 & 12 & -7 & .856 & -.997 & .147 \\
\hline 15 & 1 & -7 & .600 & .341 & -.341 \\
\hline 15 & 3 & -7 & 1.996 & 2.181 & -.18 \\
\hline 15 & 5 & -7 & 2.859 & 3.007 & -198 \\
\hline 15 & 7 & -7 & 1.605 & .991 & .014 \\
\hline 15 & 9 & -7 & .000 & .278 & -.278 \\
\hline 15 & 11 & -7 & .000 & .555 & -.555 \\
\hline 16 & 0 & -7 & .000 & .247 & -247 \\
\hline 16 & 2 & -7 & .000 & .010 & -.010 \\
\hline 16 & 4 & -7 & .429 & .663 & -.234 \\
\hline 16 & 6 & -7 & .070 & .731 & -.731 \\
\hline 16 & 8 & -7 & $.0 \mathrm{0}$ & .075 & -.076 \\
\hline 16 & 10 & -7 & .000 & -.163 & .163 \\
\hline 17 & 1 & -7 & 1.346 & 1.241 & .105 \\
\hline 17 & 3 & -7 & .666 & -.623 & -645 \\
\hline 17 & 5 & -7 & 1.383 & -1.291 & -.09 \\
\hline 17 & 7 & -7 & .000 & -.181 & .181 \\
\hline 17 & 9 & -7 & 1.121 & 1.320 & -.199 \\
\hline 18 & 0 & -7 & .60 & .264 & -.264 \\
\hline 18 & 2 & -7 & .000 & .163 & -.163 \\
\hline 18 & 4 & -7 & .000 & .672 & -.672 \\
\hline 18 & 6 & -7 & .006 & -.233 & .233 \\
\hline 19 & 1 & -7 & 100 & -.390 & .390 \\
\hline 19 & 3 & -7 & .000 & .870 & -.878 \\
\hline 0 & 0 & 8 & 2.403 & -2.553 & .15 \\
\hline 0 & 2 & 8 & .583 & -.698 & .11 \\
\hline 0 & 4 & 8 & .287 & .258 & .30 \\
\hline 0 & 6 & 8 & .578 & .531 & .047 \\
\hline 0 & 8 & 8 & .902 & -.892 & -.910 \\
\hline 0 & 10 & 8 & .765 & -.70 & -.064 \\
\hline 0 & 12 & 8 & .000 & -.923 & .923 \\
\hline 1 & 1 & 8 & .00 & -.076 & .076 \\
\hline 1 & 1 & -8 & 2.227 & 2.399 & -.71 \\
\hline 1 & 3 & 8 & .517 & .535 & -.218 \\
\hline 1 & 3 & -8 & .000 & -.631 & .631 \\
\hline 1 & 5 & 8 & .000 & -.010 & .010 \\
\hline 1 & j & -8 & .60 & -.154 & .154 \\
\hline I & 1 & 8 & .000 & .098 & -29 \\
\hline i & 7 & -8 & 1.241 & 1.709 & $-.46 ?$ \\
\hline 1 & 9 & 8 & .006 & .109 & -169 \\
\hline 1 & 9 & -8 & .832 & .858 & -.326 \\
\hline 1 & 11 & -8 & .567 & .877 & -313 \\
\hline 1 & 13 & -8 & .000 & .081 & -.581 \\
\hline 2 & 0 & 8 & 1.355 & $-1 \cdot 198$ & -.157 \\
\hline 2 & 0 & -8 & 1.128 & 1.074 & \\
\hline 2 & 2 & 8 & .000 & $-1.58 z$ & 1.58 \\
\hline
\end{tabular}




\begin{tabular}{|c|c|c|c|c|c|}
\hline & & & & & \\
\hline 2 & 2 & -8 & .000 & .298 & -298 \\
\hline$\frac{2}{2}$ & 4 & 8 & - 60. & $\begin{array}{l}-2.638 \\
-2.787\end{array}$ & $\begin{array}{l}2.638 \\
-\quad 979\end{array}$ \\
\hline 2 & 6 & 8 & .000 & -1.375 & 1.370 \\
\hline 2 & 6 & -8 & 3.324 & -2.960 & -.358 \\
\hline 2 & 8 & 8 & - บnย & $-1.85 i$ & 1.853 \\
\hline 2 & 8 & -8 &. $\operatorname{coc}$ & -.292 & .292 \\
\hline 2 & 10 & -8 & 1.848 & 1.843 & .06 \\
\hline 2 & 12 & -8 & 1.017 & -.913 & $-.1: 4$ \\
\hline 3 & 1 & 8 & .603 & -.303 & $.39 \pi$ \\
\hline 3 & 1 & -8 & - ino & -.352 & .352 \\
\hline 3 & 3 & 8 & - 0 no & .441 & -.441 \\
\hline 3 & 3 & -8 & .000 & 1.632 & -1.632 \\
\hline 3 & 5 & 8 & .000 & .193 & .198 \\
\hline 3 & 5 & -8 & 1.676 & 1.727 & -.251 \\
\hline 3 & 7 & 8 & .0 & .024 & -.224 \\
\hline 3 & 7 & -8 & .000 & .230 & -238 \\
\hline 3 & 9 & -8 & .600 & -.452 & .452 \\
\hline 3 & 11 & -8 & .784 & .824 & $-04^{4}$ \\
\hline 3 & 13 & -8 & .004 & .390 & -390 \\
\hline 4 & 0 & 8 & -205 & .375 & -.173 \\
\hline 4 & 0 & -8 & $1.360^{\circ}$ & -1.347 & -.412 \\
\hline 4 & 2 & 8 & -40 & .460 & -.466 \\
\hline 4 & 2 & -8 & .026 & .617 & -.697 \\
\hline 4 & 4 & 8 & .080 & -.329 & .329 \\
\hline 4 & 4 & -8 & .000 & 4.313 & -4.313 \\
\hline 4 & 6 & -8 & 0.06 & 2.958 & -4.958 \\
\hline 4 & 8 & -8 & .080 & -.608 & .618 \\
\hline 4 & 10 & -8 & $.02 x$ & -1.290 & 1.290 \\
\hline 钱 & 12 & -8 & .200 & $7.55 \angle$ & -1.552 \\
\hline 4 & 14 & -8 & .004 & 2.870 & -2.876 \\
\hline 5 & 1 & -8 & .969 & .917 & .0 .59 \\
\hline 5 & 3 & -8 & .458 & -.501 & .243 \\
\hline 5 & 5 & $-a$ & 1.817 & -1.815 & $-5 \frac{9}{3}$ \\
\hline 5 & 7 & -8 & .000 & -.277 & .277 \\
\hline 5 & 9 & -8 & .999 & 1.061 & -663 \\
\hline 5 & 11 & -8 & .000 & .408 & -409 \\
\hline 5 & 15 & -8 & 1.146 & -1.28. & .134 \\
\hline 5 & 15 & -8 & .000 & -.327 & $-32 ?$ \\
\hline 6 & 0 & -8 & 3.565 & $\therefore 723$ & -.153 \\
\hline 6 & 2 & -8 & 1.822 & 1.74 & .681 \\
\hline 6 & 4 & -8 & 3.472 & -5.378 & -.394 \\
\hline 6 & 6 & -8 & 1.735 & -1.575 & -.158 \\
\hline 6 & 8 & -8 & 1.357 & 1.342 & .215 \\
\hline 6 & 10 & -8 & 3.475 & 2.375 & .399 \\
\hline 6 & 12 & -8 & .650 & -.531 & .531 \\
\hline 6 & 14 & -8 & 2.163 & -1.897 & -.365 \\
\hline 7 & 1 & -8 & .976 & -1.467 & .491 \\
\hline 7 & 3 & -8 & .00 & -.111 & .111 \\
\hline 7 & 5 & -8 & 1.594 & 1.893 & $-.29 ?$ \\
\hline 7 & 7 & -8 & .200 & .355 & $-35 \bar{x}$ \\
\hline 7 & 9 & -8 & .050 & -2.215 & 2.215 \\
\hline 7 & 11 & -8 & .100 & -.714 & .7114 \\
\hline 7 & 13 & -8 & .00 & $.28 b$ & -.286 \\
\hline 7 & 15 & -8 & .600 & .744 & -.744 \\
\hline 8 & 3 & -8 & $1.7^{30}$ & $-1.55 \bar{s}$ & -.149 \\
\hline 8 & 2 & -8 & .056 & .221 & -.221 \\
\hline 8 & 4 & -8 & 3.338 & $\therefore 347$ & -.038 \\
\hline 8 & 6 & -8 & 3.196 & 3.065 & $-\mid 121$ \\
\hline 8 & $g$ & -8 & - 6 & .199 & -199 \\
\hline 8 & 10 & -8 & $2.3^{3}$ & -1.81 & -197 \\
\hline$B$ & 12 & -8 & $.3 n$ & .159 & -.159 \\
\hline
\end{tabular}




\begin{tabular}{|c|c|c|c|c|c|}
\hline 8 & 14 & -8 & 2.891 & 2.714 & .76 \\
\hline 9 & 1 & -8 & .909 & .946 & -.37 \\
\hline 9 & 3 & -8 & 1.299 & -1.378 & $\because 79$ \\
\hline 8 & 5 & -8 & 2.572 & -2.874 &.$\Xi 22$ \\
\hline 8 & $T$ & -8 & .962 & $=\therefore 076$ & .114 \\
\hline 9 & 9 & -8 & .662 & .947 & -.285 \\
\hline 8 & 11 & -8 & .665 & .683 & .319 \\
\hline 9 & 13 & -8 & .000 &.- .918 & 1.918 \\
\hline * 9 & 15 & -8 & .000 & -1.755 & 1.755 \\
\hline 10 & 0 & -8 & 1.295 & $\because 365$ & -.369 \\
\hline 10 & 2 & -8 & .256 & -.159 & -106 \\
\hline 10 & 4 & -8 & 3.855 & $-4 \cdot 128$ & 273 \\
\hline 10 & 6 & -8 & 2.840 & -3.014 & .174 \\
\hline 10 & 8 & -8 & .659 & -.671 & 612 \\
\hline 10 & 10 & -8 & 2.105 & 2.025 & .280 \\
\hline 10 & 12 & -8 & 1.077 & -1.234 & .158 \\
\hline 10 & 14 & -8 & 3.219 & -3.105 & -115 \\
\hline 11 & 1 & -8 & .713 & .812 & -.100 \\
\hline 11 & 3 & -8 & 1.021 & .977 & $-\approx 24$ \\
\hline 11 & 5 & -8 & 1.640 & 1.469 & .171 \\
\hline 11 & 7 & -8 & 2.381 & 1.403 & .678 \\
\hline 11 & 9 & -8 & .000 & -.136 & .136 \\
\hline 11 & 11 & -8 & .000 & -.196 & .196 \\
\hline 11 & 13 & -8 & 1.521 & $=456$ & .65 \\
\hline 12 & 0 & -8 & 1.903 & -1.901 & -0.02 \\
\hline$\approx 72$ & 2 & -8 & .000 & -.588 & .588 \\
\hline \#12 & 4 & -8 & .000 & 2.221 & -2.221 \\
\hline$* 12$ & 6 & -8 & .000 & .581 & -.59 \\
\hline * 12 & 8 & -8 & .000 & -1.287 & 1.267 \\
\hline$\div 12$ & 10 & -8 & .300 & -1.414 & 1.414 \\
\hline$* 12$ & 12 & -8 & .500 & .913 & -.917 \\
\hline 13 & 1 & -8 & $-90:$ & -.105 & .1 .5 \\
\hline 13 & 3 & -8 & .725 & .775 & -0.057 \\
\hline 13 & 5 & -8 & .600 & .609 & -.629 \\
\hline 13 & 7 & -8 & .000 & -.087 & .87 \\
\hline 13 & 9 & -8 & .004 & .272 & -.272 \\
\hline 13 & 11 & -8 & .200 & .486 & -.486 \\
\hline 13 & 13 & -8 & .000 & -.458 & .458 \\
\hline 14 & 0 & -8 & .026 & .235 & --35 \\
\hline 14 & 2 & -8 & .000 & -.455 & .455 \\
\hline 14 & 4 & -8 & 1.392 & -1.363 & -.129 \\
\hline 14 & 6 & -8 & .600 & .389 & -389 \\
\hline 14 & 8 & -8 & .000 & .247 & -247 \\
\hline 14 & 10 & -8 & 200 & -742 & .742 \\
\hline *14 & 12 & -8 & .200 & $-\because .052$ & 1.552 \\
\hline 15 & 1 & -8 & 1.525 & .489 &. .36 \\
\hline 15 & 3 & -8 & .676 & .864 & -.89 \\
\hline 15 & 5 & -8 & .420 & .592 & -.68 \\
\hline 15 & 7 & -8 & .568 & .919 & -.351 \\
\hline 15 & 9 & -8 & $1 .<269$ & 1.276 & -.036 \\
\hline 15 & 11 & -8 & .000 & $1.04 \mathrm{Z}$ & -1.543 \\
\hline 16 & 0 & -8 & 1.815 & $1.85^{\circ}$ & -334 \\
\hline 16 & 2 & -8 & 1.014 & .952 & .03 \\
\hline 16 & 4 & -8 & $\therefore 0$ & .413 & -.413 \\
\hline 16 & 6 & -8 & .050 & -.233 & -233 \\
\hline 16 & 8 & -8 & 1.476 & 8.321 & .155 \\
\hline 16 & 10 & -8 & 8.353 & \pm 423 & -92 \\
\hline 17 & 1 & -8 & 1.250 & -1.145 & -104 \\
\hline 17 & 3 & -8 & 1.297 & -307 & -1. \\
\hline 17 & 5 & -8 & .000 & -.617 & .617 \\
\hline 17 & 7 & -8 & .003 & $-21 \%$ & 213 \\
\hline 18 & 0 & -8 & .944 & $-.68 \%$ & -.256 \\
\hline
\end{tabular}




\begin{tabular}{|c|c|c|c|c|c|}
\hline 18 & 2 & -8 & .000 & .004 & -.004 \\
\hline 18 & 4 & -8 & .600 & .698 & -.698 \\
\hline 18 & 6 & -8 & .000 & 498 & -.498 \\
\hline 0 & 0 & 9 & 1.297 & $-4 \cdot 25$ & .128 \\
\hline 0 & 2 & 9 & .000 & .085 & -.95 \\
\hline 0 & 4 & 9 & 2.810 & 2.392 & .419 \\
\hline 0 & 6 & 9 & 2.488 & 2.319 & .69 \\
\hline v & 1 & 9 & .050 & .483 & $-48 z$ \\
\hline 1 & 1 & -9 & D. & .057 & -.057 \\
\hline 1 & 3 & 9 & .000 & .173 & -.173 \\
\hline 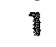 & 3 & -9 & .000 & .522 & -.522 \\
\hline 1 & 5 & 9 & .600 & -.114 & .114 \\
\hline 1 & 5 & -9 & .771 & .859 & -.287 \\
\hline 1 & 7 & -9 & .300 & .399 & -.399 \\
\hline 1 & 9 & -9 & .000 & -.224 & -224 \\
\hline 2 & 0 & -9 & 2.197 & 2.028 & .169 \\
\hline 2 & 2 & -9 & .878 & .905 & -.427 \\
\hline 2 & 4 & -9 & 1.075 & -1.125 & -55 \\
\hline 2 & 6 & -9 & 1.310 & -1.400 & .089 \\
\hline 2 & \& & -9 & .000 & .717 & -.717 \\
\hline 2 & 10 & -9 & 1.595 & 1.573 & .022 \\
\hline 3 & 1 & -9 & .000 & .366 & -.366 \\
\hline 3 & 3 & -9 & .000 & -.423 & .423 \\
\hline 3 & 5 & -9 & vol & -.761 & .761 \\
\hline 3 & 7 & -9 & .000 & .015 & -.015 \\
\hline 3 & 9 & -9 & .000 & -033 & .033 \\
\hline 3 & 11 & -9 & .000 & .003 & -203 \\
\hline 4 & 0 & -9 & .000 & -.000 & .306 \\
\hline 4 & 2 & -9 & 1.332 & 1.455 & -.124 \\
\hline 4 & 4 & -9 & 2.805 & 2.818 & -.013 \\
\hline 4 & 8 & -9 & 2.155 & 2.363 & -.205 \\
\hline 4 & 8 & -9 & .000 & .414 & -.414 \\
\hline 旃 & 10 & -9 & .000 & .558 & -558 \\
\hline 4 & 2 & -9 & 1.085 & 1.052 & .033 \\
\hline 5 & 1 & -9 & 914 & -.925 & .411 \\
\hline 5 & 3 & -9 & .000 & -.525 & .525 \\
\hline 5 & 5 & -9 & .000 & -427 & .427 \\
\hline 5 & 7 & -9 & .000 & -.178 & .179 \\
\hline 5 & 9 & -9 & .704 & -.994 & .290 \\
\hline 5 & 11 & -9 & .000 & -.706 & .736 \\
\hline 6 & 0 & -9 & 409 & -260 & -149 \\
\hline 6 & 2 & -9 & 1.022 & -.903 & -.119 \\
\hline 6 & 4 & -9 & 1.195 & -1.150 & -.045 \\
\hline 6 & 6 & -9 & .000 & -.290 & .290 \\
\hline 6 & $g$ & -9 & .639 & -511 & -128 \\
\hline 6 & 10 & -9 & 1.265 & -1.122 & -.142 \\
\hline 6 & 12 & -9 & .000 & -.515 & .515 \\
\hline 7 & 1 & -9 & .000 & -.619 & .619 \\
\hline 7 & 3 & -9 & .000 & 1.212 & -1.212 \\
\hline 7 & 5 & -9 & .000 & .544 & -.544 \\
\hline 7 & 7 & -9 & .000 & -.897 & .897 \\
\hline 7 & 8 & -9 & 100 & -.562 & .562 \\
\hline 7 & 11 & -9 & .400 & 1.122 & -1.122 \\
\hline 7 & 13 & -9 & .000 & -.077 & .077 \\
\hline 8 & 0 & -9 & .000 & .204 & -.204 \\
\hline 8 & 2 & -9 & .566 & .565 & 0.11 \\
\hline 8 & 4 & -9 & .971 & -1.079 & .157 \\
\hline 8 & 6 & -9 & .600 & .085 &.- .285 \\
\hline$g$ & 8 & -9 & .000 & -217 & .217 \\
\hline 8 & 10 & -9 & .003 & .400 & -46 \\
\hline 8 & 12 & -9 & -600 & .114 & -114 \\
\hline 9 & $\pi$ & -9 & .400 & .719 &. .719 \\
\hline
\end{tabular}




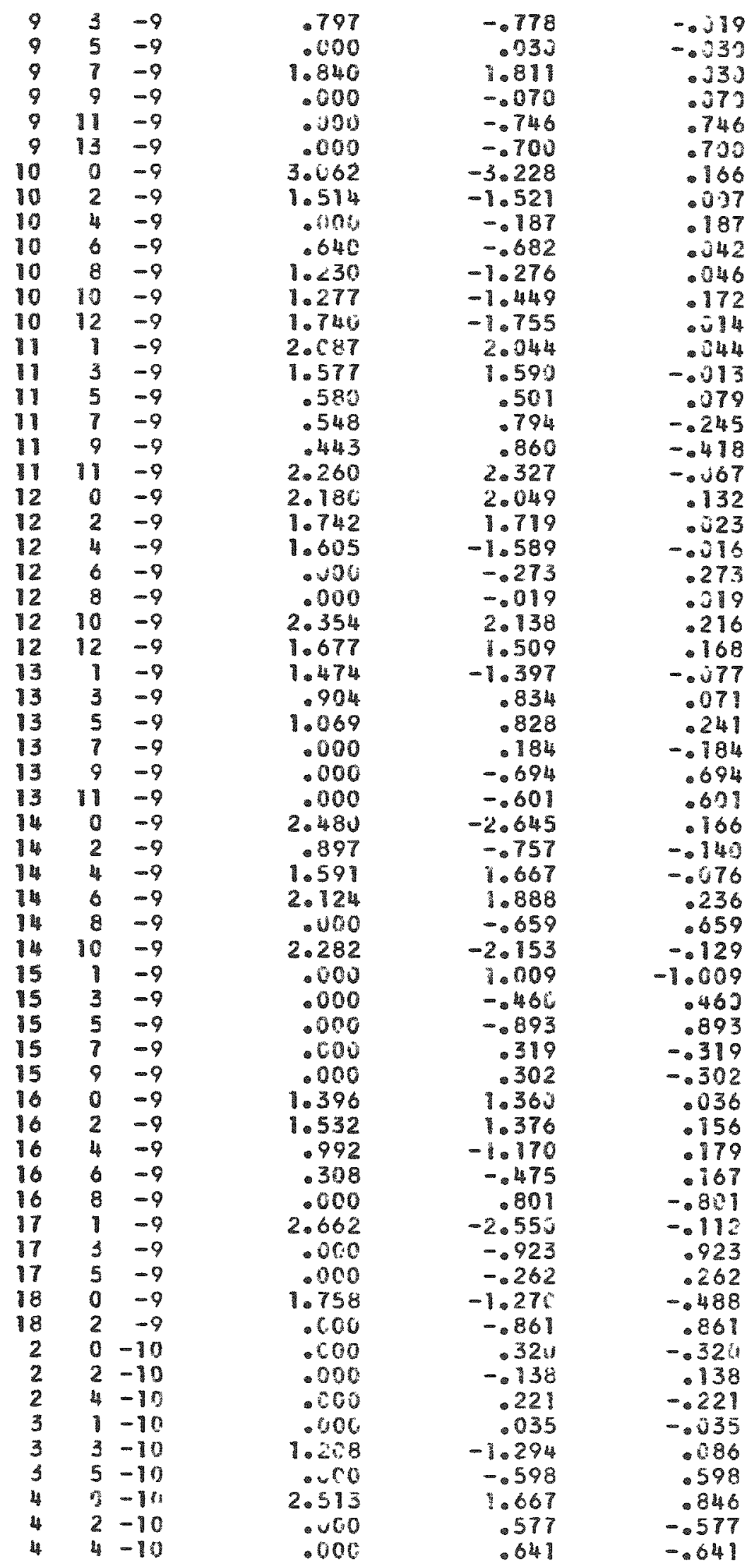




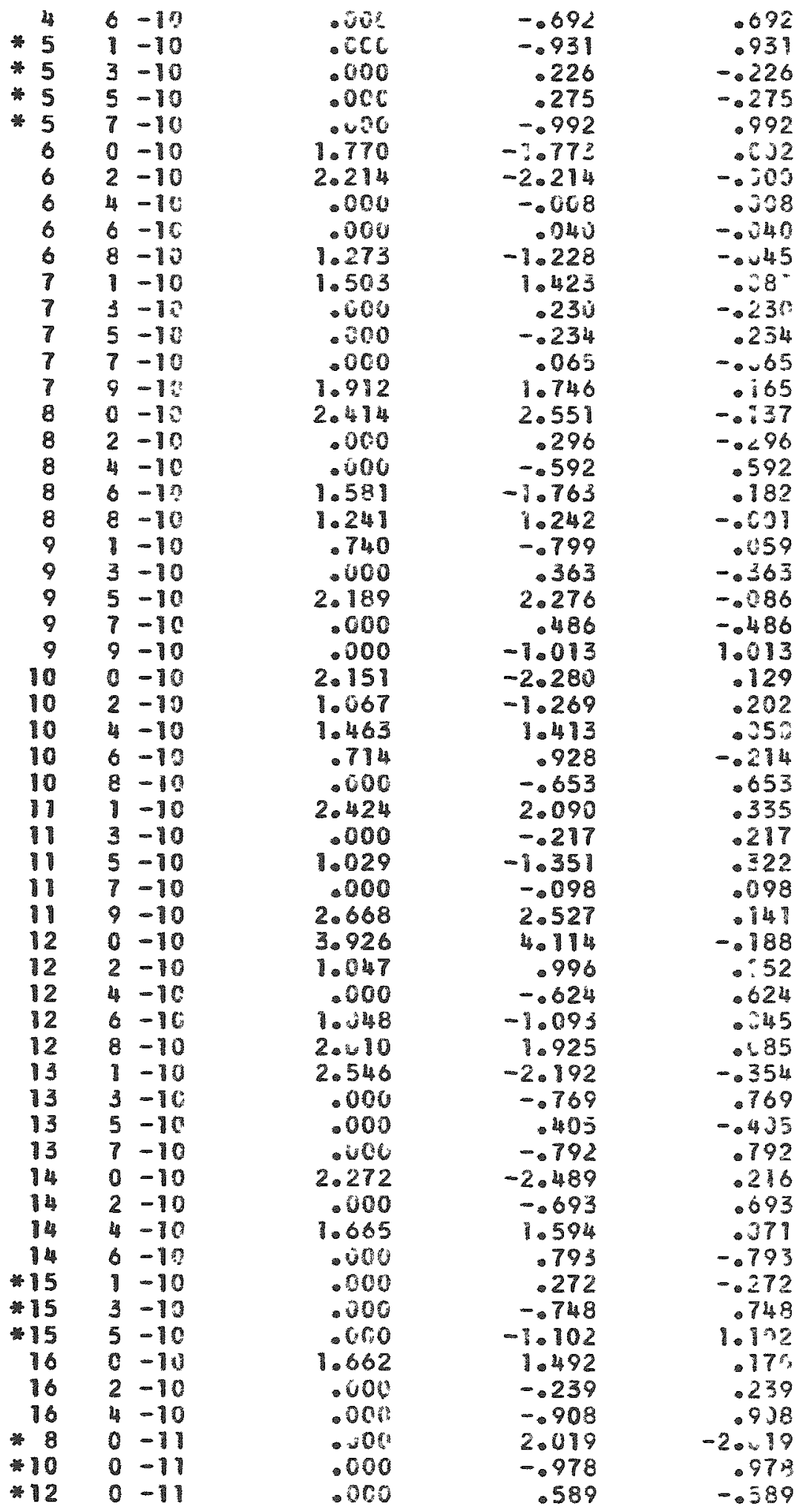


Although as indicated previously 2-dimensional Pattersons were used for a start on the structure, 3-dimensional Pattersons and 3-dimensional Fouriers gave a great deal more assistance in working out the details of the structure. For purposes of illustrating the relative positions of the atoms, we have included a 2 -dimensional hol neutron Fourier, since in this projection the overlap is not too objectionable. This projection as shown in Figure 7 shows the nitrogens to be the heavy scatterers, although all atoms with a positive neutron scattering amplitude show up reasonably strong and the hydrogens with a negative scattering amplitude are also evident. After the determination of crystal structure was quite far along, X-ray zone data was gathered by use of a small spherically shaped crystal on a singlecrystal orienter. MoK $\alpha$ radiation was used to obtain peak intensities of 126 (hol) reflections, which were converted to $F_{\text {obs }}$ values in the usual way, and the $\mathrm{X}$-ray (hol) Fourier map shown in Figure 8 was calculated. Although it is not claimed that these are the best possible $\mathrm{X}$-ray intensity data obtainable, they do indeed show, as expected, the relatively low intensities scattered from all the light atoms in comparison with the heavy $R$ u atom.

Figure 9, which is a section of the hol 3-dimensional Fourier at $y=0$, serves two purposes, namely, it illustrates the typical sharp, welldefined 3-dimensional Fourier peaks obtained at all levels, and also shows graphically the relative position of $\mathrm{HO}-\mathrm{Ru}-\mathrm{NO}$ groups. It will be recalled that their location, especially the Ru-NO bonding, was indicated as a reason for pursuing this investigation.

As indicated above, the refinement was carried out by successive $F_{0}$ and $F_{C}$ syntheses; therefore, we have included a comparison in Table VI of the atom locations based on the two Fouriers together with the leastsquares results. The location of the peaks and their heights was obtained by the use of a computer program. (16) It can be noted that there was reasonably good agreement between the three results, with the $\mathrm{H}_{2}$ and $\mathrm{H}_{3}$ atoms of the water molecule showing the greatest difference.

We were concerned about the effect of having left out the 301 reflections; therefore, we ran a Fourier using the $F_{\text {calc }}$ values with and without these reflections. The results showing coordinates and peak heights are summarized in Table VII. A slight difference can be noted in some coordinates, especially $\mathrm{H}_{1}$ and $\mathrm{H}_{2}$ atoms, but in general the agreement is quite good. 


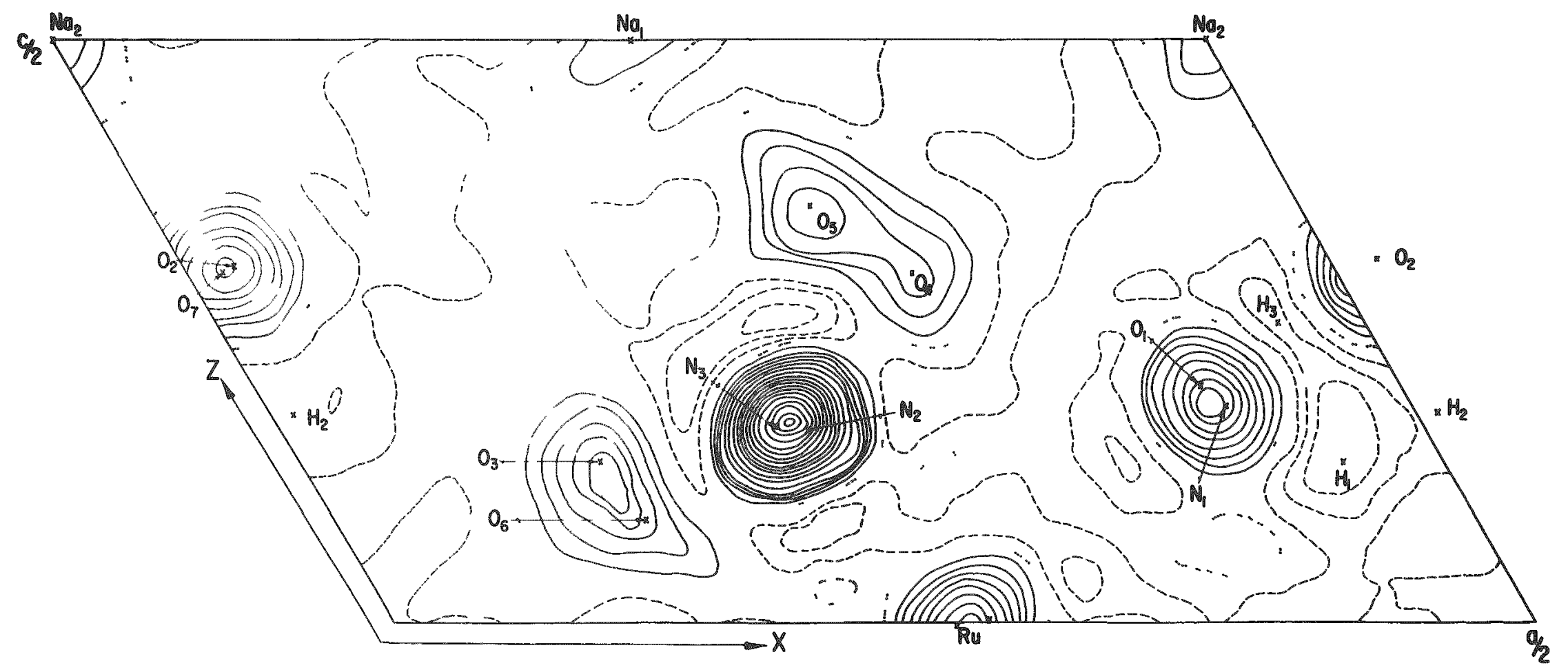

38567

Fig. 7 Neutron Fourier Showing Atom Positions on a 2-dimensional hol Projection 


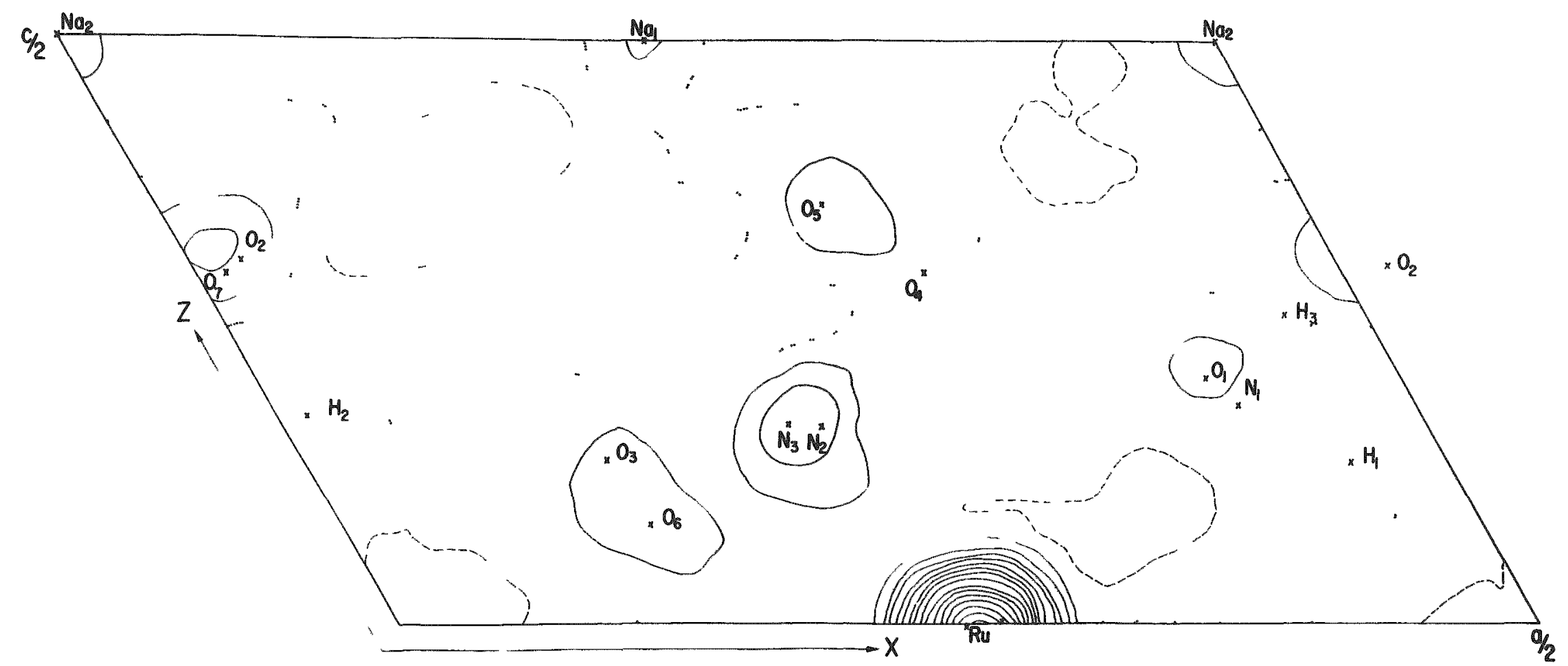

38566

Fig. 8. X-ray Fourier Showing Atom Positions on a 2-dimensional hol Projection 


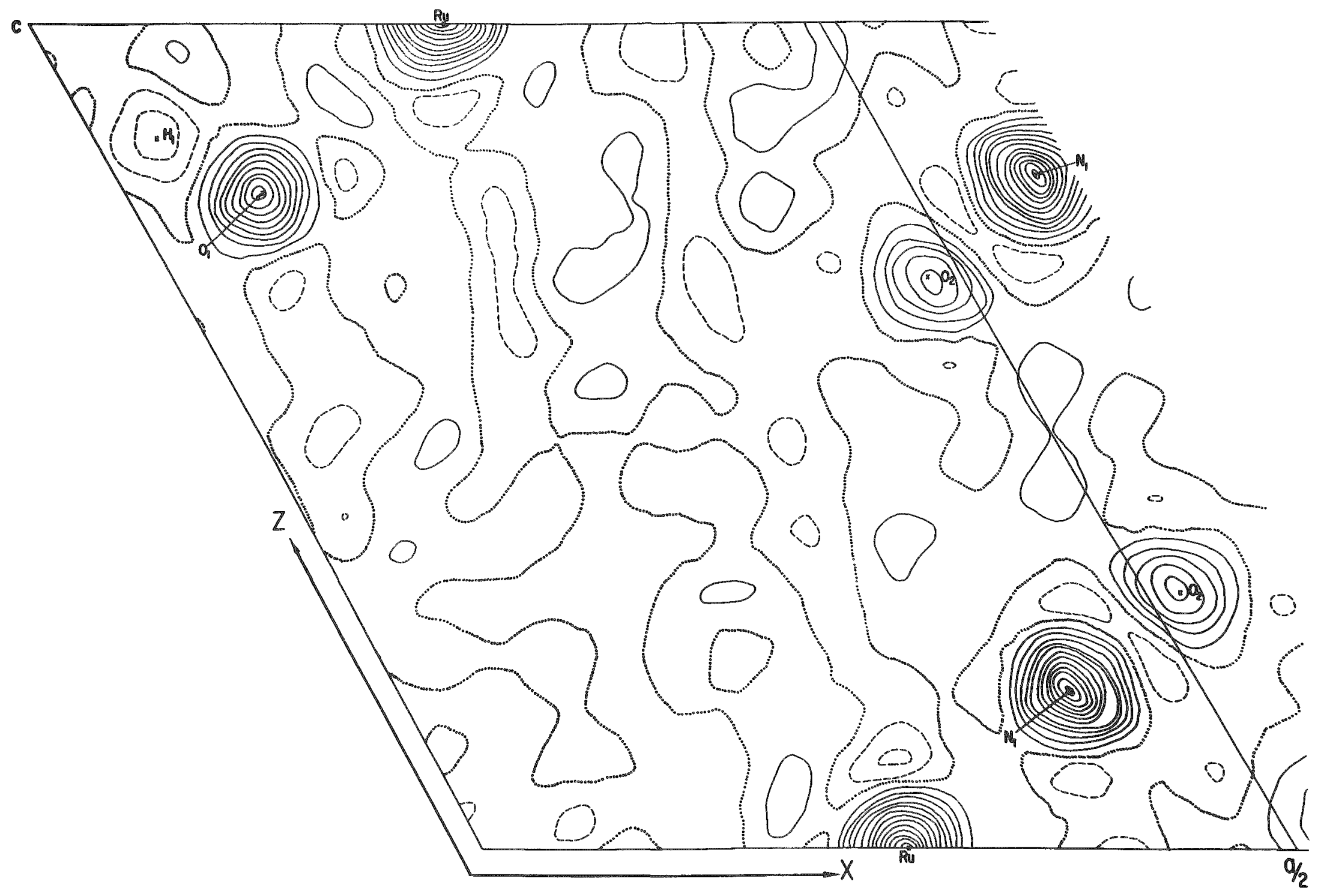


Table $\mathbb{I I}$

COMPARISON OE THE ATON LOCATIONS BASED ON F FaIC FOURIER, FóBS FOURIER,

AND THE LEAST-SQUARES RESULTS FRON THE BC REFLECTIONS

\begin{tabular}{|c|c|c|c|c|c|c|c|c|c|}
\hline \multirow{2}{*}{ Atom } & \multicolumn{3}{|c|}{ Lnit Cell Costdinates } & \multirow{2}{*}{$\begin{array}{l}\text { Peak } \\
\text { Heught }\end{array}$} & \multirow{2}{*}{ Atom } & \multicolumn{3}{|c|}{ Unit Cell Coordinates } & \multirow{2}{*}{$\begin{array}{l}\text { Peak } \\
\text { Height }\end{array}$} \\
\hline & $x$ & $y$ & $z$ & & & $\mathrm{x}$ & $y$ & $z$ & \\
\hline$H_{1}$ & $\begin{array}{r}1-0.0407 \\
2-0.0403 \\
3-0.0454\end{array}$ & $\begin{array}{l}0 \\
0 \\
0\end{array}$ & $\begin{array}{l}0.8662 \\
0.8658 \\
0.8607\end{array}$ & $\frac{1054}{1048}$ & $O_{2}$ & $\begin{array}{r}1-0.5195 \\
2-0.5199 \\
3-0.5201\end{array}$ & $\begin{array}{l}0 \\
0 \\
0\end{array}$ & $\begin{array}{l}0.3116 \\
0.3124 \\
0.3086\end{array}$ & $\begin{array}{l}2234 \\
2208\end{array}$ \\
\hline $\mathrm{H}_{2}$ & $\begin{array}{l}1-0.4847 \\
2-0.4828 \\
3-0.4871\end{array}$ & $\begin{array}{l}0.2813 \\
0.2787 \\
0.2939\end{array}$ & $\begin{array}{l}0.1739 \\
0.1730 \\
0.1800\end{array}$ & $\frac{756}{702}$ & $0_{3}$ & $\begin{array}{l}1-0.1295 \\
2-0.1295 \\
3-0.1288\end{array}$ & $\begin{array}{l}0.1088 \\
0.1081 \\
0.1104\end{array}$ & $\begin{array}{l}0.1452 \\
0.1467 \\
0.1492\end{array}$ & $\begin{array}{l}1964 \\
1977\end{array}$ \\
\hline $\mathrm{H}_{3}$ & $\begin{array}{l}1-0.4552 \\
2-0.4538 \\
3-0.4610\end{array}$ & $\begin{array}{l}0.2211 \\
0.2225 \\
0.2189\end{array}$ & $\begin{array}{l}0.2714 \\
0.2584 \\
0.2754\end{array}$ & $\frac{602}{648}$ & $0_{4}$ & $\begin{array}{l}1-0.3145 \\
2-0.3158 \\
3-03146\end{array}$ & $\begin{array}{l}0.1460 \\
0.1463 \\
0.1472\end{array}$ & $\begin{array}{l}0.3040 \\
0.3048 \\
0.3022\end{array}$ & $\begin{array}{l}2138 \\
2061\end{array}$ \\
\hline$R u$ & $\begin{array}{l}1-0.2608 \\
2-0.2608 \\
3-0.2619\end{array}$ & $\begin{array}{l}0 \\
0 \\
0\end{array}$ & $\begin{array}{l}0.0027 \\
0.0027 \\
0.0032\end{array}$ & $\begin{array}{l}5636 \\
5575\end{array}$ & $0_{5}$ & $\begin{array}{l}1-0.2098 \\
2-0.2100 \\
3-0.2118\end{array}$ & $\begin{array}{l}0.1105 \\
0.1106 \\
0.1116\end{array}$ & $\begin{array}{l}0.6373 \\
0.6370 \\
0.6380\end{array}$ & $\begin{array}{l}2687 \\
2660\end{array}$ \\
\hline$N_{1}$ & $\begin{array}{l}1-0.4212 \\
2-0.4210 \\
3-0.4205\end{array}$ & $\begin{array}{l}0 \\
0 \\
0\end{array}$ & $\begin{array}{l}0.1916 \\
0.1911 \\
0.1906\end{array}$ & $\begin{array}{l}6058 \\
6018\end{array}$ & .6 & $\begin{array}{l}1-0.3944 \\
2-0.3622 \\
3-0.3675\end{array}$ & $\begin{array}{l}0.1566 \\
0.1591 \\
0.1559\end{array}$ & $\begin{array}{l}0.9122 \\
0.9121 \\
0.9143\end{array}$ & $\begin{array}{l}1623 \\
1586\end{array}$ \\
\hline $\mathrm{N}_{2}$ & $\begin{array}{l}1-0.2305 \\
2-0.2298 \\
3-0.2313\end{array}$ & $\begin{array}{l}0.1007 \\
0.1011 \\
0.1002\end{array}$ & $\begin{array}{l}0.1720 \\
0.1704 \\
0.1716\end{array}$ & $\begin{array}{l}6188 \\
6055\end{array}$ & $0_{7}$ & $\begin{array}{l}1-0.0110 \\
2-0.0118 \\
3-0.0106\end{array}$ & $\begin{array}{l}0.2321 \\
0.2319 \\
0.2307\end{array}$ & $\begin{array}{l}0.3022 \\
0.3033 \\
0.2999\end{array}$ & $\begin{array}{l}2390 \\
2466\end{array}$ \\
\hline $\mathrm{N}_{3}$ & $\begin{array}{l}1-0.2834 \\
2-0.2835 \\
3-0.2839\end{array}$ & $\begin{array}{l}0.1024 \\
0.1024 \\
0.1024\end{array}$ & $\begin{array}{l}0.8277 \\
0.8278 \\
0.8284\end{array}$ & $\begin{array}{l}6522 \\
6390\end{array}$ & $\mathrm{Na}$ & $\begin{array}{ll}1- & 0 \\
2- & 0 \\
3- & 0\end{array}$ & $\begin{array}{l}0.1106 \\
0.1109 \\
0.1089\end{array}$ & $\begin{array}{l}0.5900 \\
0.5000 \\
0.5000\end{array}$ & $\begin{array}{l}1930 \\
1948\end{array}$ \\
\hline $\mathrm{o}_{1}$ & $\begin{array}{l}1-0.0859 \\
2-0.0857 \\
3-0.0877\end{array}$ & $\begin{array}{l}0 \\
0 \\
0\end{array}$ & $\begin{array}{l}0.7918 \\
0.7918 \\
0.7907\end{array}$ & $\begin{array}{l}3945 \\
3806\end{array}$ & $\mathrm{Na}_{2}$ & $\begin{array}{l}1-0.2500 \\
2-0.2500 \\
3-0.2500\end{array}$ & $\begin{array}{l}0.2500 \\
0.2500 \\
0.2500\end{array}$ & $\begin{array}{l}0.5000 \\
0.5000 \\
0.5000\end{array}$ & $\begin{array}{l}1525 \\
1472\end{array}$ \\
\hline
\end{tabular}

"Noie: 1 - Results fram Falc Fourver.

2 - Results from $F_{0 h s}$ Fourier.

3 - Results from last least-sọuares cycle based on BC reflections.

Table III

EFFECT OF THE MISSING REFLECTONS AS INOICATED BY A COMPARISOS OF ATOM LOGATIONS

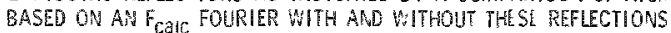

\begin{tabular}{|c|c|c|c|c|c|c|c|c|c|}
\hline \multirow{2}{*}{ Atom } & \multicolumn{3}{|c|}{ Unt Cell Coordinates } & \multirow{2}{*}{$\begin{array}{l}\text { Peak } \\
\text { Height }\end{array}$} & \multirow{2}{*}{ Atom } & \multicolumn{3}{|c|}{ UniE Cell Coordinates } & \multirow{2}{*}{$\begin{array}{l}\text { Peak } \\
\text { Height }\end{array}$} \\
\hline & $\mathrm{x}$ & $y$ & 2 & & & $x$ & y & $z$ & \\
\hline $\mathrm{H}_{2}$ & $\begin{array}{l}1=0.5083 \\
2=0.4884 \\
3-0.5086\end{array}$ & $\begin{array}{l}0.2909 \\
0.2781 \\
0.2920\end{array}$ & $\begin{array}{l}0.1841 \\
0.1840 \\
0.1811\end{array}$ & $\frac{685}{858}$ & $o_{3}$ & $\begin{array}{l}1-0.1303 \\
2-0.1298 \\
3-0.1288\end{array}$ & $\begin{array}{l}0.1098 \\
0.1084 \\
0.1094\end{array}$ & $\begin{array}{l}0.1430 \\
0.1465 \\
0.1420\end{array}$ & $\begin{array}{l}2667 \\
2134\end{array}$ \\
\hline $\mathrm{H}_{3}$ & $\begin{array}{l}1-0.4653 \\
2-0.4668 \\
3-0.4631\end{array}$ & $\begin{array}{l}0.2201 \\
0.2200 \\
0.2190\end{array}$ & $\begin{array}{l}0.2727 \\
0.2679 \\
0.2675\end{array}$ & $\frac{\overline{943}}{600}$ & $o_{4}$ & $\begin{array}{l}1-0.3132 \\
2-0.3133 \\
3-0.3146\end{array}$ & $\begin{array}{l}0.1472 \\
0.1465 \\
0.1474\end{array}$ & $\begin{array}{l}0.3002 \\
0.3012 \\
0.3020\end{array}$ & $\begin{array}{l}2769 \\
2243\end{array}$ \\
\hline$R_{L i}$ & $\begin{array}{l}1-0.2625 \\
2-0.2613 \\
3-0.2023\end{array}$ & $\begin{array}{l}0 \\
0 \\
0\end{array}$ & $\begin{array}{l}0.0039 \\
0.0024 \\
00037\end{array}$ & $\begin{array}{l}6829 \\
5947\end{array}$ & $0_{5}$ & $\begin{array}{l}1-0.2131 \\
2-0.2104 \\
30.2123\end{array}$ & $\begin{array}{l}0.1115 \\
0.1113 \\
0.1118\end{array}$ & $\begin{array}{l}0.6406 \\
0.6393 \\
0.6391\end{array}$ & $\begin{array}{l}3092 \\
2737\end{array}$ \\
\hline 82 & $\begin{array}{l}1-0.2306 \\
2-0.2302 \\
3-0.2311\end{array}$ & $\begin{array}{l}0.1005 \\
0.1005 \\
0.1002\end{array}$ & $\begin{array}{l}0.1716 \\
01718 \\
0.1719\end{array}$ & $\begin{array}{l}6886 \\
62,4\end{array}$ & $0_{7}$ & $\begin{array}{l}1-0.0116 \\
2-0.0110 \\
3-0.0120\end{array}$ & $\begin{array}{l}0.2302 \\
0.2309 \\
0.2304\end{array}$ & $\begin{array}{l}0.3010 \\
0.3023 \\
0.3016\end{array}$ & $\begin{array}{l}2787 \\
2455\end{array}$ \\
\hline$N_{3}$ & $\begin{array}{l}1-0.2165 \\
2-0.2168 \\
3-0.2165\end{array}$ & $\begin{array}{l}0.3973 \\
0.3972 \\
0.3977\end{array}$ & $\begin{array}{l}0.1731 \\
0.1729 \\
0.1723\end{array}$ & $\begin{array}{l}7159 \\
0563\end{array}$ & $\mathrm{Nag}_{1}$ & $\begin{array}{ll}1 & 0 \\
2- & 0 \\
3- & 0\end{array}$ & $\begin{array}{l}0.1091 \\
0.1001 \\
0.1097\end{array}$ & $\begin{array}{l}0.5000 \\
0.5000 \\
0.5000\end{array}$ & $\begin{array}{l}2103 \\
1923\end{array}$ \\
\hline$o_{1}$ & $\begin{array}{l}1-0.0866 \\
2-0.0857 \\
3-0.0875\end{array}$ & $\begin{array}{l}0 \\
0 \\
0\end{array}$ & $\begin{array}{l}0.7904 \\
0.7910 \\
0.7904\end{array}$ & $\begin{array}{l}4621 \\
4095\end{array}$ & $\mathrm{~N}_{2}$ & $\begin{array}{l}1-0.2500 \\
2-0.2500 \\
3-0.2500\end{array}$ & $\begin{array}{l}0.2500 \\
0.2500 \\
0.2500\end{array}$ & $\begin{array}{l}0.5000 \\
0.5000 \\
0.5900\end{array}$ & $\begin{array}{l}1611 \\
1469\end{array}$ \\
\hline
\end{tabular}

- Make: 1 - Results from Faic Fouriar Lasted an all reflections

2 - Results frome $F_{\text {calc }}$ Fourer after removing m.ssing reflections.

3 - Results from last leasti-squaras cycle based on ABC reflections. 


\section{DISCUSSION OF THE STRUCTURE}

Since there are eight general positions in $\mathrm{C} 2 / \mathrm{m}$ and only four molecules per unit cell, the atoms are distributed over both special and general

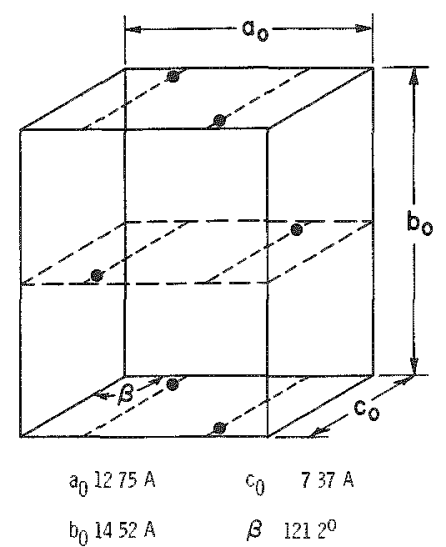

$106-7082$

Fig. 10. Lattice Constants and Schematic Structure of $\mathrm{Na}_{2}\left[\mathrm{Ru}\left(\mathrm{NO}_{2}\right)_{4} \mathrm{NO} \cdot \mathrm{OH}\right] 2 \mathrm{H}_{2} \mathrm{O}$ positions. The ruthenium, nitrosyl, and hydroxyl are in special positions on the mirror plane; the sodiums occupy two sets of special positions, one on a center of symmetry and one on a two-fold axis; and the nitrito groups and waters are in general positions. The relative locations of the $R u$ atoms within the unit cell are shown in Figure 10, where it is evident that the $\mathrm{Ru}$ atoms are very close to $1 / 4,0,0$ and equivalent positions.

The structure can be envisioned as consisting mainly of 6-fold coordination about three central atoms, namely, the $\mathrm{Ru}, \mathrm{Na} \mathrm{a}_{1}$, and $\mathrm{Na}$ forming three different kinds of octahedra which include most of the atoms within the structure. These octahedra form an interlocking chain throughout the structure, in which there are common atoms or common edges shared between the octahedra, as shown in Figures 11 and 12. Each of the three octahedra have relatively the same orientation within the cell. Four atoms in each are arranged around a central atom in a near coplanar square which is perpendicular or nearly perpendicular to the mirror plane or parallel to the $b$ axis. The

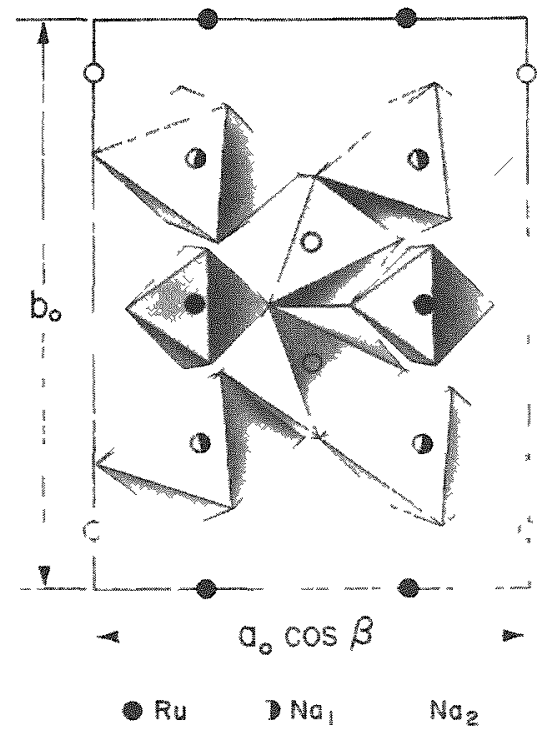

$106-7627$

Fig. 11. Octahedra Viewed Down the $c$ Axis

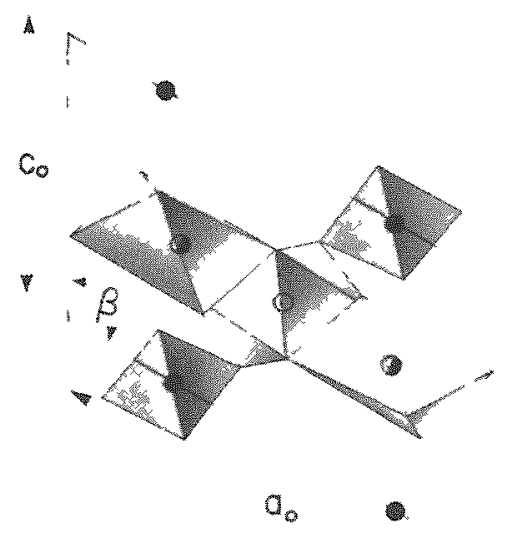

$106-7628$

Fig. 12. Octahedra Vlewed Down the $b$ Axis 
other two atoms involved in the 6-fold coordination group lie along a line perpendicular or nearly perpendicular to this square plane and nearly parallel to the mirror plane, thus forming the octahedra. In order to better envisage this structure a model consisting of two complete unit cells (100 atoms per unit cell) was constructed of small plastic balls positioned on rods parallel to the "b" axis.

\section{A. Configuration about the Ruthenium}

Four nitrogens of the nitrito groups, the nitrogen of the nitrosyl, and the oxygen of the hydroxyl form a 6 -fold coordination about the ruthe-

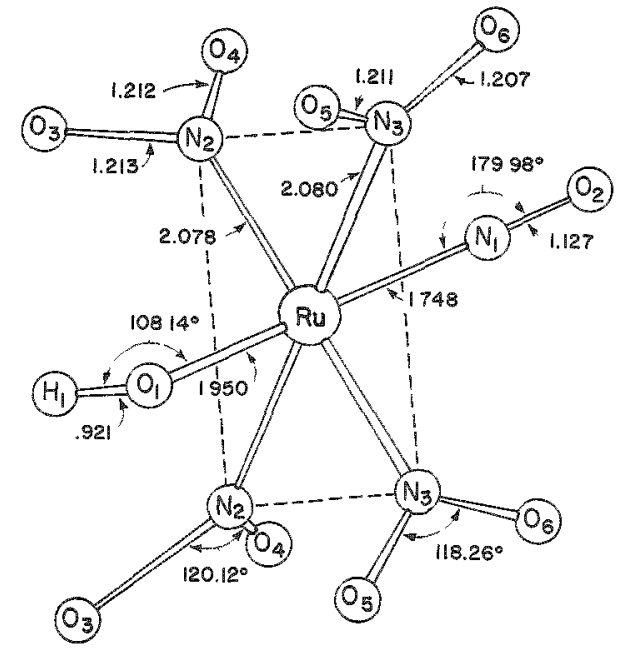

$106-7630$

Fig. 13. Configuration about the Ruthenium Atom nium (see Figure 13). The nitrito nitrogens form a near square, which is coplanar and nearly perpendicular to the mirror plane with ruthenium in the center. Each Ru-N bond forms a $45^{\circ}$ angle with the mirror. The $\mathrm{NO}$ and $\mathrm{OH}$ groups lie in the mirror, trans with respect to the ruthenium, and the $N_{1}$ of the nitrosyl and the $O_{1}$ of the hydroxyl are at the apices of the octahedron.

The distances from the central $R u$ atom to its nearest neighbors are shown in Table VIIIA1. The two unique Ru to $N$ of the nitrito distances, $\mathrm{Ru}-\mathrm{N}_{2}$ and $\mathrm{Ru}-\mathrm{N}_{3}$, are essentially equal, being 2.078 and $2.080 \AA$, respectively, which agree well with the sum of Pauling's radii. The Ru to $N$ of the nitrosyl is much shorter, being $1.748 \AA$, and the $R u-N-O$ bond is linear, since an angle of $179.98^{\circ}$ was found, suggesting that

the electronic configuration is $R u=\dot{N}=\ddot{O}$ : and that $R u-\ddot{N}=\ddot{O}$ : makes no contribution. This Ru-N distance of $1.748 \AA$ is considerably shorter than the $1.85 \AA$ reported $(5)$ for the $\mathrm{Ru}-\mathrm{N}$ distance in $\mathrm{K}_{2}\left[\mathrm{RuNO}(\mathrm{OH})\left(\mathrm{NO}_{2}\right)_{4}\right]$. The $R u$ to $O$ of the hydroxyl is $1.950 \AA$, very close to the sum of Pauling's octahedral covalent radii.

The very nearly square nature of the four nitrogens $\left(2-\mathrm{N}_{2}\right.$ and $\left.2-\mathrm{N}_{3}\right)$ about the $R u$ is indicated by the $N_{2}-N_{2}, N_{3}-N_{3}$ and $N_{2}-N_{3}$ distances of 2.909, 2.972 , and $2.937 \AA$, respectively. A link connecting $N_{1}$ and $O_{1}$ makes an angle of $90.33^{\circ}$ with the plane of the square. The Ru octahedron contains very little distortion as evidenced by the angle and distances indicated above, and by the almost equal edge lengths from the apex atoms $\mathrm{N}_{1}$ and $\mathrm{O}_{1}$ to the coplanar nitrogens $\mathrm{N}_{2}$ and $\mathrm{N}_{3}$, as shown in Table VIII A3. The plane shown dashed in Figure 13 is parallel to the $y$ axis and is rotated $11.2^{\circ}$ counterclockwise from the $\mathrm{z}$ axis. The $\mathrm{N}-\mathrm{O}$ distance in the nitrosyl is $1.127 \AA$, which is in good agreement with $1.1 \AA$ reported by Bokii, Ang-nu, and Khodashova $(5)$ for $\mathrm{K}_{2}\left[\mathrm{RuNO}(\mathrm{OH})\left(\mathrm{NO}_{2}\right)\right]$, but the angle of $179.98^{\circ}$ is in 
Table VIII

SOME INTER - AND INTRAMOLECULAR BOND DISTANCES $(1)$ AND ANGLES

A. Configuration about the Ruthenium

Number of

Distances

Atom $1 \quad$ Atom 2

Distance,

1. Ru to closest neighbors (6-fold coordination)

$\begin{array}{llll}1 & \mathrm{Ru} & \mathrm{N}_{1}(\mathrm{NO}) & 1.748(4) \\ 2 & \mathrm{Ru} & \mathrm{N}_{2}\left(\mathrm{NO}_{2}\right)_{2} & 2.078(3) \\ 2 & \mathrm{Ru} & \mathrm{N}_{3}\left(\mathrm{NO}_{2}\right)_{3} & 2.080(3) \\ 1 & \mathrm{Ru} & \mathrm{O}_{1}(\mathrm{OH}) & 1.950(5)\end{array}$

2. Nitrogen "square" about Ru

$\begin{array}{llll}1 & \mathrm{~N}_{3}\left(\mathrm{NO}_{2}\right)_{3} & \mathrm{~N}_{3}\left(\mathrm{NO}_{2}\right)_{3} & 2.972(4) \\ 2 & \mathrm{~N}_{2}\left(\mathrm{NO}_{2}\right)_{2} & \mathrm{~N}_{3}\left(\mathrm{NO}_{2}\right)_{3} & 2.937(3) \\ 1 & \mathrm{~N}_{2}\left(\mathrm{NO}_{2}\right)_{2} & \mathrm{~N}_{2}\left(\mathrm{NO}_{2}\right)_{2} & 2.909(4)\end{array}$

3. Ru octahedron edges

$\begin{array}{llll}2 & \mathrm{~N}_{1}(\mathrm{NO}) & \mathrm{N}_{2}\left(\mathrm{NO}_{2}\right)_{2} & 2.754(3) \\ 2 & \mathrm{~N}_{1}(\mathrm{NO}) & \mathrm{N}_{3}\left(\mathrm{NO}_{2}\right)_{3} & 2.744(3) \\ 2 & \mathrm{O}_{1}(\mathrm{OH}) & \mathrm{N}_{2}\left(\mathrm{NO}_{2}\right)_{2} & 2.831(4) \\ 2 & \mathrm{O}_{1}(\mathrm{OH}) & \mathrm{N}_{3}\left(\mathrm{NO}_{2}\right)_{2} & 2.798(4)\end{array}$

4. Vicinity of the Ru octahedron

$\begin{array}{llll}1 & \mathrm{~N}_{1}(\mathrm{NO})_{1} & \mathrm{O}_{2}(\mathrm{NO}) & 1.127(7) \\ 2 & \mathrm{~N}_{2}\left(\mathrm{NO}_{2}\right)_{2} & \mathrm{O}_{3}\left(\mathrm{NO}_{2}\right)_{2} & 1.213(5) \\ 2 & \mathrm{~N}_{2}\left(\mathrm{NO}_{2}\right)_{2} & \mathrm{O}_{4}\left(\mathrm{NO}_{2}\right)_{2} & 1.212(5) \\ 2 & \mathrm{~N}_{3}\left(\mathrm{NO}_{2}\right)_{3} & \mathrm{O}_{5}\left(\mathrm{NO}_{2}\right)_{3} & 1.211(4) \\ 2 & \mathrm{~N}_{3}\left(\mathrm{NO}_{2}\right)_{3} & \mathrm{O}_{6}\left(\mathrm{NO}_{2}\right)_{3} & 1.207(5) \\ 1 & \mathrm{H}_{1}(\mathrm{OH}) & \mathrm{O}_{1}(\mathrm{OH}) & 0.921(9) \\ & & & 0.974(11)^{*} \\ & & & 1.038(10)^{* *}\end{array}$

5. Angles - Ru octahedron

\begin{tabular}{cccr} 
Atom 1 & Atom 2 & Atom 3 & Angle, deg \\
\cline { 1 - 2 } & $\mathrm{N}_{1}(\mathrm{NO})$ & $\mathrm{O}_{2}(\mathrm{NO})$ & $179.98(55)$ \\
$\mathrm{Ru}$ & $\mathrm{O}_{1}(\mathrm{OH})$ & $\mathrm{H}_{1}(\mathrm{OH})$ & $108.15(67)$ \\
$\mathrm{N}_{1}(\mathrm{NO})$ & $\mathrm{Ru}$ & $\mathrm{O}_{1}(\mathrm{OH})$ & $178.68(26)$ \\
$\mathrm{O}_{3}\left(\mathrm{NO}_{2}\right)$ & $\mathrm{N}_{2}\left(\mathrm{NO}_{2}\right)$ & $\mathrm{O}_{4}\left(\mathrm{NO}_{2}\right)$ & $120.12(37)$ \\
$\mathrm{O}_{5}\left(\mathrm{NO}_{2}\right)$ & $\mathrm{N}_{3}\left(\mathrm{NO}_{2}\right)$ & $\mathrm{O}_{6}\left(\mathrm{NO}_{2}\right)$ & $118.26(37)$ \\
$\mathrm{N}_{2}\left(\mathrm{NO}_{2}\right)$ & $\mathrm{Ru}$ & $\mathrm{N}_{2}\left(\mathrm{NO}_{2}\right)$ & $8888(17)$
\end{tabular}

(1) Standard errors $\left(\mathrm{x} 10^{3}\right)$ appear in parentheses. 
Table VIII (Contd.)

\begin{tabular}{|c|c|c|c|}
\hline Atom 1 & Atom 2 & Atom 3 & Angle, deg \\
\hline $\mathrm{N}_{2}\left(\mathrm{NO}_{2}\right)$ & $\mathrm{Ru}$ & $\mathrm{N}_{3}\left(\mathrm{NO}_{2}\right)$ & $89.88(8)$ \\
\hline $\mathrm{N}_{3}\left(\mathrm{NO}_{3}\right)$ & $\mathrm{Ru}$ & $\mathrm{N}_{3}\left(\mathrm{NO}_{2}\right)$ & 91.36 (I1) \\
\hline $\mathrm{N}_{1}(\mathrm{NO})$ & Ru. & $\mathrm{N}_{2}\left(\mathrm{NO}_{2}\right)$ & $91.75(15)$ \\
\hline $\mathrm{O}_{1}(\mathrm{OH})$ & Ru & $\mathrm{N}_{2}\left(\mathrm{NO}_{2}\right)$ & $89.24(15)$ \\
\hline
\end{tabular}

B. Configuration about the Sodium-1

Number of

Distances

Atom 1 Atom 2

Distance, $\AA$

1. $\mathrm{Na}_{1}$ to closest neighbors (6-fold coordination)

$\begin{array}{llll}2 & \mathrm{Na}_{1} & \mathrm{O}_{4}\left(\mathrm{NO}_{2}\right)_{2} & 2.505(4) \\ 2 & \mathrm{Na}_{1} & \mathrm{O}_{5}\left(\mathrm{NO}_{2}\right)_{3} & 2.413(4) \\ 2 & \mathrm{Na}_{1} & \mathrm{O}_{7}\left(\mathrm{H}_{2} \mathrm{O}\right) & 2.613(6)\end{array}$

2. Oxygen "square" about $\mathrm{Na}_{1}$

$\begin{array}{llll}2 & \mathrm{O}_{4}\left(\mathrm{NO}_{2}\right)_{2} & \mathrm{O}_{5}\left(\mathrm{NO}_{2}\right)_{3} & 3.392(7) \\ 2 & \mathrm{O}_{4}\left(\mathrm{NO}_{2}\right)_{2} & \mathrm{O}_{5}\left(\mathrm{NO}_{2}\right)_{3} & 3.562(8)\end{array}$

3. $\mathrm{Na}_{1}$ octahedron edges

$\begin{array}{llll}2 & \mathrm{O}_{7}\left(\mathrm{H}_{2} \mathrm{O}\right) & \mathrm{O}_{4}\left(\mathrm{NO}_{2}\right)_{2} & 3.140(7) \\ 2 & \mathrm{O}_{7}\left(\mathrm{H}_{2} \mathrm{O}\right) & \mathrm{O}_{4}\left(\mathrm{NO}_{2}\right)_{2} & 4.043(7) \\ 2 & \mathrm{O}_{7}\left(\mathrm{H}_{2} \mathrm{O}\right) & \mathrm{O}_{5}\left(\mathrm{NO}_{2}\right)_{3} & 3.014(6) \\ 2 & \mathrm{O}_{7}\left(\mathrm{H}_{2} \mathrm{O}\right) & \mathrm{O}_{5}\left(\mathrm{NO}_{2}\right)_{3} & 3.552(8)\end{array}$

4. Vicinity of the $\mathrm{Na}_{1}$ octahedron
2
$\mathrm{Na}_{1}$
$\mathrm{O}_{3}\left(\mathrm{NO}_{2}\right)_{2}$
$3.049(5)$
2
$\mathrm{Na}_{1}$
$\mathrm{O}_{6}\left(\mathrm{NO}_{2}\right)_{3}$
$2.957(6)$

C. Configuration about the Sodium-2

1. $\mathrm{Na}_{2}$ to closest neighbors (6-fold coordination)
2
2
2
$\mathrm{Na}_{2}$
$\mathrm{O}_{1}(\mathrm{OH})$
$2.430(7)$
$\mathrm{O}_{7}\left(\mathrm{H}_{2} \mathrm{O}\right)$
$2.335(8)$
$\mathrm{Na}_{2}$
$\mathrm{O}_{5}\left(\mathrm{NO}_{2}\right)_{3}$
$2.345(5)$

2. Oxygen "square" about $\mathrm{Na}_{2}$

$\begin{array}{llll}1 & \mathrm{O}_{1}(\mathrm{OH}) & \mathrm{O}_{1}(\mathrm{OH}) & 3.669(8) \\ 2 & \mathrm{O}_{1}(\mathrm{OH}) & \mathrm{O}_{7}\left(\mathrm{H}_{2} \mathrm{O}\right) & 3.518(6) \\ 1 & \mathrm{O}_{7}\left(\mathrm{H}_{2} \mathrm{O}\right) & \mathrm{O}_{7}\left(\mathrm{H}_{2} \mathrm{O}\right) & 3.088(9)\end{array}$


Table VIII (Contd.)

3. $\mathrm{Na}_{2}$ octahedron edges

\begin{tabular}{lll} 
Atom 1 & Atom 2 & Distance, \\
\cline { 3 - 3 } $\mathrm{O}_{5}\left(\mathrm{NO}_{2}\right)_{3}$ & $\mathrm{O}_{7}\left(\mathrm{H}_{2} \mathrm{O}\right)$ & $3.014(6)$ \\
$\mathrm{O}_{5}\left(\mathrm{NO}_{2}\right)_{3}$ & $\mathrm{O}_{7}\left(\mathrm{H}_{2} \mathrm{O}\right)$ & $3.552(8)$ \\
$\mathrm{O}_{5}\left(\mathrm{NO}_{2}\right)_{3}$ & $\mathrm{O}_{1}(\mathrm{OH})$ & $2.875(6)$ \\
$\mathrm{O}_{5}\left(\mathrm{NO}_{2}\right)_{3}$ & $\mathrm{O}_{1}(\mathrm{OH})$ & $3.839(6)$
\end{tabular}

D. Bond Distances and Angles between O-O (Possible Hydrogen Bonding)

\begin{tabular}{|c|c|c|c|c|}
\hline Atom 1 & Atom 2 & Atom 3 & 1-3 Distance & 1-2-3 Angle \\
\hline $\mathrm{O}_{7}\left(\mathrm{H}_{2} \mathrm{O}\right)$ & & $\mathrm{O}_{1}(\mathrm{OH})$ & $3,518(6)$ & \\
\hline $\mathrm{O}_{7}\left(\mathrm{H}_{2} \mathrm{O}\right)$ & & $\mathrm{O}_{2}(\mathrm{NO})$ & $3.916(5)$ & \\
\hline $\mathrm{O}_{7}\left(\mathrm{H}_{2} \mathrm{O}\right)$ & $\mathrm{H}_{2}\left(\mathrm{H}_{3}\right)$ & $\mathrm{O}_{3}\left(\mathrm{NO}_{2}\right)$ & $2.919(7)$ & $139^{\circ}\left(29^{\circ}\right)$ \\
\hline $\mathrm{O}_{7}\left(\mathrm{H}_{2} \mathrm{O}\right)$ & $\mathrm{H}_{2}\left(\mathrm{H}_{3}\right)$ & $\mathrm{O}_{3}\left(\mathrm{NO}_{2}\right)$ & $3.308(9)$ & $131^{\circ}\left(63^{\circ}\right)$ \\
\hline $\mathrm{O}_{7}\left(\mathrm{H}_{2} \mathrm{O}\right)$ & $\mathrm{H}_{2}\left(\mathrm{H}_{3}\right)$ & $\mathrm{O}_{4}\left(\mathrm{NO}_{2}\right)$ & $3.079(7)$ & $43^{\circ}\left(145^{\circ}\right)$ \\
\hline $\mathrm{O}_{7}\left(\mathrm{H}_{2} \mathrm{O}\right)$ & $\mathrm{H}_{2}\left(\mathrm{H}_{3}\right)$ & $\mathrm{O}_{4}\left(\mathrm{NO}_{2}\right)$ & $3.140(7)$ & $46^{\circ}\left(81^{\circ}\right)$ \\
\hline $\mathrm{O}_{7}\left(\mathrm{H}_{2} \mathrm{O}\right)$ & $\mathrm{H}_{2}\left(\mathrm{H}_{3}\right)$ & $\mathrm{O}_{5}\left(\mathrm{NO}_{2}\right)$ & $3.014(6)$ & $59^{\circ}\left(63^{\circ}\right)$ \\
\hline $\mathrm{O}_{7}\left(\mathrm{H}_{2} \mathrm{O}\right)$ & $\mathrm{H}_{2}\left(\mathrm{H}_{3}\right)$ & $\mathrm{O}_{5}\left(\mathrm{NO}_{2}\right)$ & $3.552(8)$ & $78^{\circ}\left(23^{\circ}\right)$ \\
\hline $\mathrm{O}_{7}\left(\mathrm{H}_{2} \mathrm{O}\right)$ & $\mathrm{H}_{2}\left(\mathrm{H}_{3}\right)$ & $\mathrm{O}_{6}\left(\mathrm{NO}_{2}\right)$ & $2.972(8)$ & $103^{\circ}\left(119^{\circ}\right)$ \\
\hline $\mathrm{O}_{7}\left(\mathrm{H}_{2} \mathrm{O}\right)$ & $\mathrm{H}_{2}\left(\mathrm{H}_{3}\right)$ & $\mathrm{O}_{6}\left(\mathrm{NO}_{2}\right)$ & $3.187(7)$ & $96^{\circ}\left(80^{\circ}\right)$ \\
\hline $\mathrm{O}_{7}\left(\mathrm{H}_{2} \mathrm{O}\right)$ & & $\mathrm{O}_{7}\left(\mathrm{H}_{2} \mathrm{O}\right)$ & $3.088(9)$ & \\
\hline $\mathrm{O}_{3}\left(\mathrm{NO}_{2}\right)$ & $\mathrm{H}_{1}$ & $O_{1}(O H)$ & $2.842(6)$ & $135^{\circ}$ \\
\hline $\mathrm{O}_{3}\left(\mathrm{NO}_{2}\right)$ & $\mathrm{H}_{1}$ & $\mathrm{O}_{1}(\mathrm{OH})$ & $3.433(7)$ & $110^{\circ}$ \\
\hline $\mathrm{O}_{5}\left(\mathrm{NO}_{2}\right)$ & $\mathrm{H}_{1}$ & $\mathrm{O}_{1}(\mathrm{OH})$ & $2.875(6)$ & \\
\hline $\mathrm{O}_{3}\left(\mathrm{NO}_{2}\right)$ & & $\mathrm{O}_{3}\left(\mathrm{NO}_{2}\right)$ & $2.838(10)$ & \\
\hline $\mathrm{O}_{3}\left(\mathrm{NO}_{2}\right)$ & & $\mathrm{O}_{3}\left(\mathrm{NO}_{2}\right)$ & $3.175(14)$ & \\
\hline & & $\mathrm{O}_{2}(\mathrm{NO})$ & & \\
\hline
\end{tabular}

E. $\mathrm{H}_{2} \mathrm{O}$ Distances and Angle

\begin{tabular}{|c|c|c|c|}
\hline Atom 1 & Atom 2 & Atom 3 & Distance Angle \\
\hline $\mathrm{H}_{2}$ & & $\mathrm{O}_{7}\left(\mathrm{H}_{2} \mathrm{O}\right)$ & $\begin{array}{l}0.929(16) \\
1.061(19) * \\
1.185(18) * *\end{array}$ \\
\hline $\mathrm{H}_{3}$ & & $\mathrm{O}_{7}\left(\mathrm{H}_{2} \mathrm{O}\right)$ & $\begin{array}{l}0.911(14) \\
1.000(15)^{*} \\
1.130(14)^{* *}\end{array}$ \\
\hline $\mathrm{H}_{2}\left(\mathrm{H}_{2} \mathrm{O}\right)$ & & $\mathrm{H}_{3}\left(\mathrm{H}_{2} \mathrm{O}\right)$ & $1.500(26)$ \\
\hline $\mathrm{H}_{2}$ & $O_{7}$ & $\mathrm{H}_{3}$ & $109.3 \pm 1.9^{\circ}$ \\
\hline
\end{tabular}

*Assumed that $\mathrm{H}$ rides on $\mathrm{O}$.

**Assumed that the $\mathrm{H}$ and $\mathrm{O}$ move independently. 
disagreement with their repoxted value of $\sim 170^{\circ}$. The $\mathrm{Ru}-\mathrm{O}_{1}-\mathrm{H}_{1}$ angle is $108.15^{\circ}$ and the $\mathrm{O}-\mathrm{H}$ distance is $0.921 \mathrm{~A}$ as calculated with parameters averaged over temperature motion. A $0.974 \AA$ distance is found if the hy drogen is assumed to ride on the oxygen and a $1.038 \AA$ distance if the two atoms are assumed to move independently. The $O_{1}-R u-N_{1}$ angle is $178.68^{\circ}$. The N-O distances and angles of the nitrito groups are in good agreement with accepted values, with the following distances obtained for the four unique $\mathrm{N}-\mathrm{O}$ bonds: $1.213,1.212,1.211$, and $1.207 \AA$. The two $\mathrm{O}-\mathrm{N}-\mathrm{O}$ angles are $120.12^{\circ}$ and $118.26^{\circ}$.

B. Configuration about the Sodium - 1

The 6-fold coordination about the $\mathrm{Na}_{1}$ consists of 6 oxygen atoms, four from $\mathrm{NO}_{2}$ groups and two from water molecules, as shown in Figure 14.

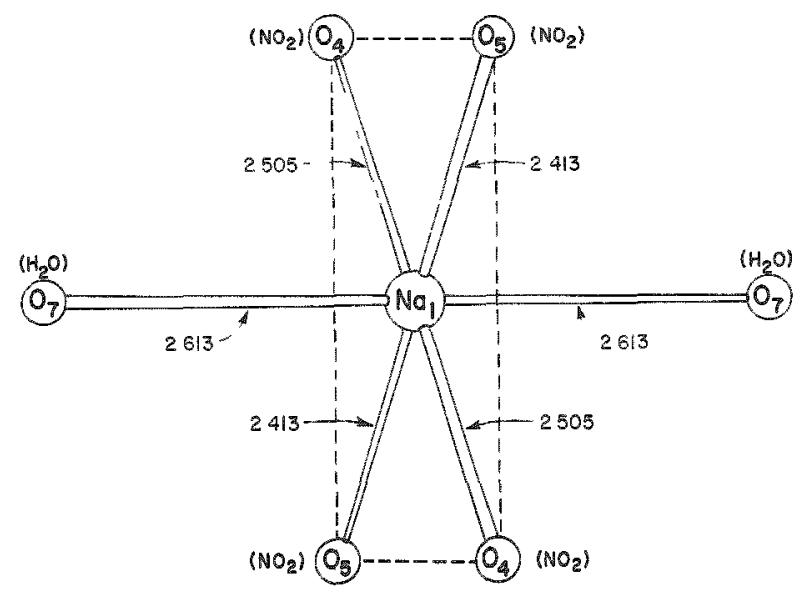

$106-7629-A$

Fig. 14. Configuration about the Sodium-1 Atom The four oxygens of the $\mathrm{NO}_{2}$ groups are coplanar and are arranged in a parallelogram with the $\mathrm{Na}_{1}$ at the center, since the $\mathrm{Na}_{1}$ is at a center of symmetry. This parallelogram, shown dashed, is tilted approximately $1.1^{\circ}$ from the $y$ axis and is rotated approximately $19.7^{\circ}$ counter clockwise from the $z$ axis. Oxygens from each of two water molecules are at the apices of the octahedron, which has approximately the same relative orientation as the Ru octahedron described above.

The distances from the central $\mathrm{Na}_{1}$ atom to its nearest oxygen neighbors are as follows: 2 at $2.413 \AA$ and 2 at $2.505 \AA$ from the two different $\mathrm{NO}_{2}$ groups; and 2 at $2.613 \AA$ from the waters. The average $\mathrm{Na}-\mathrm{O}$ distance of the above, namely, $2.510 \AA$, is within the $2.25-2.78 \AA$ range reported $(17)$ for 6 -fold coordination from 16 previous determinations and only slightly higher than the $2.44 \AA$ average reported for these same compounds.

The two sets of O-O distances along the edges of the distorted square, namely, 3.392 and $3.562 \AA$, indicate the extent of the distortion to a parallelogram. A line through $\mathrm{O}_{7}-\mathrm{Na}_{1}-\mathrm{O}_{7}$ forms an angle of approximately $68^{\circ}$ to the parallelogram and is tilted approximately $6.3^{\circ}$ from the ac face. The distortion of the octahedron is evident by the different edge lengths from the apex atoms, $O_{7}$, to the coplanax oxygen atoms, $O_{4}$ and $O_{5}$, as shown in Table VIIIB3. 
It is also interesting to note that there are four other oxygen atoms fairly close to the $\mathrm{Na}_{1}$. These are two $\mathrm{O}_{3}\left(\mathrm{NO}_{2}\right)$ at $3.049 \AA$ and two $\mathrm{O}_{6}\left(\mathrm{NO}_{2}\right)$ at $2.957 \AA$ which are not a part of the three basic octahedra - $\mathrm{Ru}, \mathrm{Na}_{1}$, or $\mathrm{Na}_{2}$. Further comments will be made about these later.

C. Configuration about the Sodium-2

The 6-fold coordination about the $\mathrm{Na}_{2}$ consists of six oxygen atoms: two from hydroxyls, two from nitritos, and two from waters, as shown in

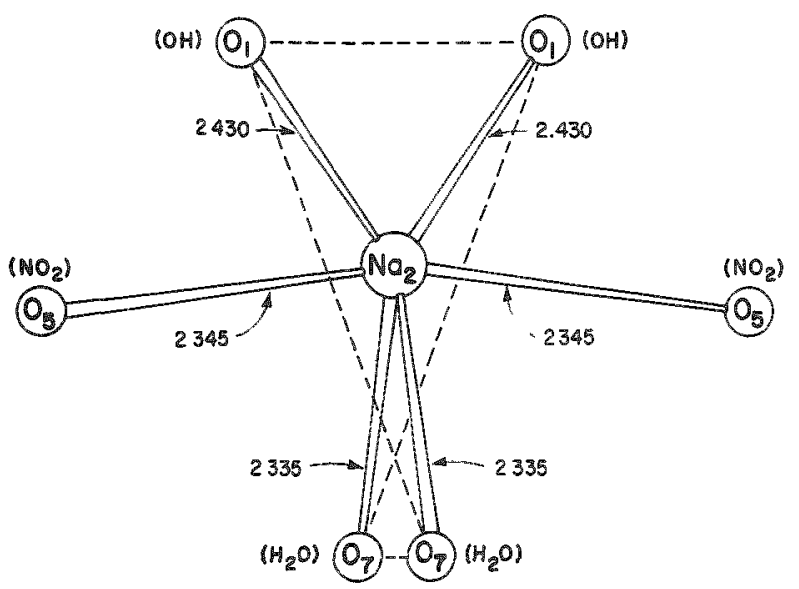

38890

Fig. 15. Configuration about the Sodium-2 Atom

Figure 15. Two oxygens from hydroxyls and two from waters are arranged in a greatly distorted. square about a nearly central $\mathrm{Na}_{2}$ atom. Because the $\mathrm{Na}_{2}$ is on a twofold axis it is not required to be at the center of this square. Two $\mathrm{O}_{5}$ from nitritos are at the apices of the octahedron.

The distances from the central $\mathrm{Na}_{2}$ atom to its nearest oxygen neighbors are as follows: two $\mathrm{O}_{1}$ (OH) at $2.430 \AA$; two $\mathrm{O}_{7}\left(\mathrm{H}_{2} \mathrm{O}\right)$ at $2.335 \AA$; two $\mathrm{O}_{5}\left(\mathrm{NO}_{2}\right)$ at $2.345 \AA$. The average distance, $2.370 \AA$, is somewhat lower than the average $2.510 \AA$ found for the $\mathrm{Na}_{1}$ octahedron above and also is even lower than the average $2.44 \AA$ reported (17) previously for the 16 compounds. However, the average of all $12 \mathrm{Na}-\mathrm{O}$ distances is, coincidentally, $2.44 \AA$.

In the case of the $\mathrm{Na}_{2}$ octahedron the two $\mathrm{O}_{1}^{\prime} \mathrm{s}(\mathrm{OH})$ and two $\mathrm{O}_{7} \mathrm{~s}^{\mathrm{s}}\left(\mathrm{H}_{2} \mathrm{O}\right)$ form a greatly distorted square as indicated by the sides of $3.669 \AA, 2$ of $3.518 \AA$, and $3.088 \AA$. The plane of this square is also greatly twisted as indicated by the fact that the $O_{3}$ and $O_{7}$ atoms lie approximately $0.46 \AA$ and $0.51 \AA$, respectively, from the least-squares plane containing the $O_{1}, O_{7}$, and $\mathrm{Na}_{2}$ atoms. The normal of this plane makes an angle of $7.5^{\circ}$ with the line joining the $\mathrm{O}_{5}-\mathrm{O}_{5}$ and is parallel to the ac plane. The least-squares plane is rotated $13.5^{\circ}$ counterclockwise from the $\mathrm{z}$ axis. The skewed nature of this $\mathrm{Na}_{2}$ octahedron is also evident from the variation of the edge lengths, ranging from 2.875 to $3.552 \AA$ as shown in Table VIIIC 3 .

\section{General Features of the Structure}

It is interesting to examine the structure of this compound on the basis of the octahedron-octahedron linkage as shown in Figures 11 and 12 . The Ru octahedra are linked to the $\mathrm{Na}_{2}$ octahedra through a common $\mathrm{O}_{1}$ atom; the $\mathrm{Na}_{1}$ octahedra are linked to $\mathrm{Na}_{2}$ octahedra by a common edge 
formed by $\mathrm{O}_{5}-\mathrm{O}_{7}$ atoms; the $\mathrm{Na}_{2}$ octahedrons are linked to other $\mathrm{Na}_{2}$ octahedrons by a common edge of $\mathrm{O}_{1}-\mathrm{O}_{1}$. This arrangement leaves all octahedra interconnected. The only atoms not included in these octahedra are: $\mathrm{H}_{1}(\mathrm{OH}), \mathrm{O}_{2}(\mathrm{NO}), \mathrm{H}_{2}$ and $\mathrm{H}_{3}\left(\mathrm{H}_{2} \mathrm{O}\right)$, and $\mathrm{O}_{3}$ and $\mathrm{O}_{6}\left(\mathrm{NO}_{2}\right)$.

Each Ru octahedron may also be viewed as being surrounded and separated from other Ru octahedra by sheets of $\mathrm{Na}$ atoms and $\mathrm{H}_{2} \mathrm{O}$ molecules as follows:

(1) a sheet parallel to the (001) plane at $z=1 / 2$ contains only $\mathrm{Na}_{1}$ and $\mathrm{Na}_{2}$ atoms;

(2) sheets parallel to the (010) plane at $y=1 / 4$ and $3 / 4$ contain $\mathrm{Na}_{1}$ atoms and $\mathrm{O}_{7}$ atoms of the water molecules, and

(3) sheets parallel to the (100) plane at $x=0$ and $1 / 2$ contain $\mathrm{Na}_{2}$ and $\mathrm{O}_{7}$ atoms of the water molecules.

Thus, it seems reasonable that the structure could indeed physically fall apart, as it did under low humidity conditions, if water is removed and the $\mathrm{Na}-\mathrm{O}$ bonds are broken.

It is also of interest to determine the possible role of hydrogen bonding in the structure. In Table VIIID the various $O-O$ distances are given that might be involved in hydrogen bonding. All of the $\mathrm{O}_{7}\left(\mathrm{H}_{2} \mathrm{O}\right)-\mathrm{O}$ distances appear to be rather large, although several fall within the range from 2.49 to $3.15 \AA$ reported (18) for 16 different inorganic salts containing water. The shortest $\mathrm{O}_{7}\left(\mathrm{H}_{2} \mathrm{O}\right)$-O distances are observed with the $\mathrm{O}_{3}$ and $\mathrm{O}_{6}$, which have distances of 2.919 and $2.972 \AA$, respectively. These are the two oxygens which, as described before, are not involved with the 6 -fold coordination about $\mathrm{Na}_{1}$ or $\mathrm{Na}_{2}$, and hence might be involved in a weak hy drogen bond. A hydrogen bond might be involved between $\mathrm{O}_{7}-\mathrm{H}_{2}-\mathrm{O}_{3}$, which has an angle of $139^{\circ}$ and a distance of $2.919 \AA$, and $\mathrm{O}_{7}-\mathrm{H}_{3}-\mathrm{O}_{6}$, which has an angle of $119^{\circ}$ and a distance of $2.972 \AA$. These angles, however, are rather small in terms of an average of $167^{\circ}$ found for 15 compounds as recently reported. (19) Perhaps even more probable is an $\mathrm{O}_{3}-\mathrm{H}_{1}-\mathrm{O}_{1}$ hydrogen bond, which involves a slightly closer distance, $2.842 \AA$, and an angle of $135^{\circ}$. The relative different environment between the $\mathrm{O}_{3}$ and $\mathrm{O}_{6}$ as shown above may indeed make the $\mathrm{NO}_{2}$ groups about the $\mathrm{Ru}$ somewhat different: $2-\mathrm{NO}_{2}$ groups involving $\mathrm{O}_{3}$ and $2-\mathrm{NO}_{2}$ involving $\mathrm{O}_{6}$.

The various distances observed in the water molecule are summarized in Table VIIIE. Distances of 0.929 and $0.911 \AA$ were observed for $\mathrm{O}-\mathrm{H}$ bonds of the two hydrogens calculated with parameters averaged over temperature motion. These distances increased to 1.061 and $1.000 \AA$ if it was assumed that the $H$ rides on the $O$ and somewhat greater distances if the $\mathrm{H}$ and $O$ move independently. The $\mathrm{H}-\mathrm{O}-\mathrm{H}$ angle is $109.3^{\circ}$. Both 
distances and angle agree favorably with other recent results $(20)$ in which a value of approximately $1.0 \AA$ for the $\mathrm{O}-\mathrm{H}$ distance was observed and an angle of approximately $110^{\circ}$.

Calculations have also been made for the RMS radial thermal dis placement and the RMS component along each principal axis. The results are shown in Table IX. The hydrogens, as might be expected, have large radial displacements, that of the water hydrogens being greater than that of the hydroxyl hydrogens. The $\mathrm{O}_{3}{ }^{\prime} \mathrm{s}$ and $\mathrm{O}_{6}$ 's appear to have the largest displacements among the oxygens, which may be attributed to the fact that, as explained previously, these two oxygens seem to participate less in the structural bonding. The $\mathrm{Na}_{1}$, which is located in the octahedron having the longest $\mathrm{Na}-\mathrm{O}$ bonds, also has a large displacement. The heavy atom Ru has the smallest displacement and is essentially isotropic. The RMS radial thermal displacements are in agreement with the peak heights, listed in Table VI, of the Fobs Fourier map. Thus, among atoms of a given kind, the ones having the smallest displacements have the largest peak heights.

Table IX

RMS RADIAL THERMAL DISPLACEMENT AND THE RMS COMPONENT ALONG EACH PRINCIPAL AXIS

\begin{tabular}{|c|c|c|c|c|}
\hline \multirow{2}{*}{$\frac{\text { Atom }}{\mathrm{H}_{1}(\mathrm{OH})}$} & \multirow{2}{*}{$\begin{array}{c}\text { RMS Radial, } \\
\AA\end{array}$} & \multicolumn{3}{|c|}{$\begin{array}{l}\text { RMS Component along the } \\
\text { Three Principal Axes, }\end{array}$} \\
\hline & & $0.150(11)$ & $0.203(10)$ & $0.342(15)$ \\
\hline $\mathrm{H}_{2}\left(\mathrm{H}_{2} \mathrm{O}\right)$ & $0.647(17)$ & $0.216(14)$ & $0.393(21)$ & $0.467(24)$ \\
\hline $\mathrm{H}_{3}\left(\mathrm{H}_{2} \mathrm{O}\right)$ & 0.575 (13) & $0.195(12)$ & $0.361(16)$ & $0.403(17)$ \\
\hline $\mathrm{Ru}$ & 0.255 (3) & $0.143(4)$ & $0.149(4)$ & $0.149(5)$ \\
\hline $\mathrm{N}_{1}(\mathrm{NO})$ & $0.305(3)$ & $0.154(4)$ & $0.180(3)$ & $0.191(4)$ \\
\hline $\mathrm{N}_{2}\left(\mathrm{NO}_{2}\right)$ & $0.312(2)$ & $0.145(3)$ & $0.186(3)$ & $0.204(3)$ \\
\hline $\mathrm{N}_{3}\left(\mathrm{NO}_{2}\right)$ & 0.301 (2) & $0.146(3)$ & $0.184(2)$ & $0.187(3)$ \\
\hline $\mathrm{O}_{1}(\mathrm{OH})$ & $0.289(4)$ & $0.153(6)$ & $0.162(6)$ & $0.185(6)$ \\
\hline $\mathrm{O}_{2}(\mathrm{NO})$ & $0.416(6)$ & $0.155(7)$ & $0.270(8)$ & $0.276(8)$ \\
\hline $\mathrm{O}_{3}\left(\mathrm{NO}_{2}\right)$ & $0.439(5)$ & $0.158(6)$ & $0.244(5)$ & $0.329(7)$ \\
\hline $\mathrm{O}_{4}\left(\mathrm{NO}_{2}\right)$ & $0.407(4)$ & $0.161(5)$ & $0.230(5)$ & $0.294(6)$ \\
\hline $\mathrm{O}_{5}\left(\mathrm{NO}_{2}\right)$ & $0.389(4)$ & $0.151(5)$ & $0.212(5)$ & $0.290(6)$ \\
\hline $\mathrm{O}_{6}\left(\mathrm{NO}_{2}\right)$ & $0.517(8)$ & $0.156(6)$ & $0.214(6)$ & $0.443(10)$ \\
\hline $\mathrm{O}_{7}\left(\mathrm{H}_{2} \mathrm{O}\right)$ & $0.400 \quad(4)$ & $0.180(5)$ & $0.214(5)$ & $0.285(6)$ \\
\hline $\mathrm{Na}_{1}$ & $0.434(12)$ & $0.162(12)$ & $0.179(11)$ & $0.360(15)$ \\
\hline $\mathrm{Na}_{2}$ & $0.358(8)$ & $0.167(10)$ & $0.176(10)$ & $0.264(11)$ \\
\hline Av. & 0.404 & 0.162 & 0.222 & 0.291 \\
\hline
\end{tabular}




\section{ACKNOWLEDGEMENTS}

We wish to express our appreciation to Prof. G. W. Watt, Department of Chemistry, The University of Texas for calling attention to the interest in this problem, and to Drs. H. Pollock and R. M. Wallace of the Savannah River Laboratory, E. I. du Pont de Nemours and Co., for supplying the large single crystals. We also acknowledge the tremendous help received from the various members of the Diffraction Group in the gathering of the data and to members of the Applied Mathematics Division who assisted with the computer techniques. Special thanks are due to H. Knott of the Diffraction Group for the construction of the crystal model which was invaluable in the analysis and description of this structure. The authors also wish to express appreciation to F. G. Foote and S. S. Sidhu for encouragement to carry out this program.

\section{REEERENCES}

1. A. McL. Mathieson, D. P. Mellor, and N. C. Stephenson, Acta Cryst., 5, 185 (1952).

2. C. S. Adams and D. P. Mellor, Aust. J. of Sci. Research, Series A, 5, $577(1952)$.

3. G. B. Bokii and N. A. Parpiev, Kristallographia, 2, 691 (1957).

4. N. A. Parpiev and M. A. Porai-Koshits, Kristallographia, 4, 30 (1959).

5. G. B. Bokii, Wang Ang-nu and T. S. Khodashova, Zhurnal Strukturnoi Khimii, 3 , No. 2, 163 (1962).

6. International Union of Crystallography Sixth International Congress, Abstracts, Rome, Italy, September 1963, p. A34.

7. S. H. Simonsen and M. H. Mueller, The Crystal Structure of Disodium Tetranitritonitrosohydroxy ruthenate(III) 2-Hydrate by Neutron Diffraction, submitted to Journal of Inorganic and Nuclear Chemistry, March 1964.

8. E. I. du Pont de Nemours and Co., Explosives Dept., Atomic Energy Division, Savannah River Laboratory. Private Communication.

9. M. H. Mueller, L. Heaton, S. S. Sidhu, and J. Terandy, Argonne Dual Neutron Diffractometers Using a Single Primary Beam, ANL-6537 (Nov 1962). 
10. E. R. Howells, D. C. Phillips, and D. Rogers, Acta Cryst., 3, 210 (1950).

11. Singelfungen-Dormstadt-Pittsburgher Fourier Program for Projection as Modified by R. Shiono, Chemistry Dept., University of Pittsburgh.

12. W. G. Sly and D. P. Shoemaker, MIFRI: Two and Three-Dimensional Crystallographic Fourier Summation Program for the IBM-704 Computer, Dept. of Chemistry, Massachusetts Institute of Technology, Cambridge, Massachusetts.

13. M. J. Buerger, Vector Space, John Wiley and Sons, Inc., N. Y. (1959).

14. B. R. Penfold, Patterson Superposition Program, Harvard University (March 1960).

15. W. R. Busing and H. A. Levy, A Crystallographic Least Squares Refinement Program for the IBM-704, CF-59-4-37 (1959).

16. M. H. Mueller, F. Clark, and S. H. Simonsen, Program for the Location of Fourier Peak Centers, ANL-6699 (July 1963).

17. International Tables for X-ray Crystallography, Vol. III, Chapt. 4.1, 258, The Kynoch Press (1962).

18. Ibid.。p. 273.

19. W. C. Hamilton, J. of Phys. Soc. of Japan, 17, Supp1. B-II, p. 374 (1962).

20. H. G. Smith, Private Communication. 